\title{
Chosen Ciphertext Secure Keyed-Homomorphic Public-Key Encryption *
}

\author{
Keita Emura ${ }^{\dagger} \quad$ Goichiro Hanaoka Koji Nuida $^{\ddagger} \quad$ Go Ohtake \\ Takahiro Matsudall Shota Yamada**
}

September 15, 2017

\begin{abstract}
In homomorphic encryption schemes, anyone can perform homomorphic operations, and therefore, it is difficult to manage when, where and by whom they are performed. In addition, the property that anyone can "freely" perform the operation inevitably means that ciphertexts are malleable, and it is well-known that adaptive chosen ciphertext (CCA) security and the homomorphic property can never be achieved simultaneously. In this paper, we show that CCA security and the homomorphic property can be simultaneously handled in situations that the user(s) who can perform homomorphic operations on encrypted data should be controlled/limited, and propose a new concept of homomorphic publickey encryption, which we call keyed-homomorphic public-key encryption (KH-PKE). By introducing a secret key for homomorphic operations, we can control who is allowed to perform the homomorphic operation. To construct KH-PKE schemes, we introduce a new concept, transitional universal property, and present a practical KH-PKE scheme with multiplicative homomorphic operations from the decisional Diffie-Hellman (DDH) assumption. For $\ell$-bit security, our DDH-based KH-PKE scheme yields only $\ell$-bit longer ciphertext size than that of the Cramer-Shoup PKE scheme. Finally, we consider an identitybased analogue of KH-PKE, called keyed-homomorphic identity-based encryption (KH-IBE) and give its concrete construction from the Gentry IBE scheme.
\end{abstract}

Keywords: homomorphic public key encryption, CCA2 security, hash proof system

\section{Introduction}

\subsection{Background and Motivation}

In homomorphic encryption schemes, homomorphic operations can be performed on encrypted plaintexts without decrypting the corresponding ciphertexts. Owing to this attractive property, several homomorphic public key encryption (PKE) schemes have been proposed [18, 24, 40]. Furthermore, fully homomorphic encryption (FHE) that allows a homomorphic operation with respect to any circuit, has been proposed by Gentry [23]. This has had a resounding impact not only in the cryptographic research community, but also in the business community. One of the reasons for such a big impact is that FHE is suitable for ensuring security

\footnotetext{
*An extended abstract appears in the 16th International Conference on Practice and Theory in Public Key Cryptography (PKC 2013) [19]. This is the full version.

${ }^{\dagger}$ National Institute of Information and Communications Technology (NICT), Japan. k-emura@nict.go.jp

$\ddagger$ National Institute of Advanced Industrial Science and Technology (AIST), Japan. hanaoka-goichiro@aist.go.jp

$\S$ National Institute of Advanced Industrial Science and Technology (AIST)/JST PRESTO, Japan. k.nuida@aist.go.jp

I Japan Broadcasting Corporation, Japan. ohtake.g-fw@nhk .or .jp

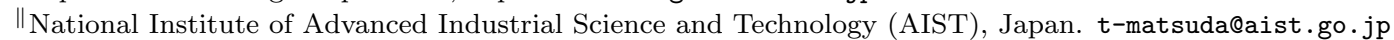

** National Institute of Advanced Industrial Science and Technology (AIST), Japan. yamada-shota@aist.go.jp
} 
in cloud environments (e.g., encrypted data stored in a database can be updated without any decryption procedure).

Improvement in the security of homomorphic encryption will lead to wider deployment of cloud-type applications, whereas the property that anyone can "freely" perform homomorphic operations inevitably means that ciphertexts are malleable. Therefore, it is well-known that adaptive chosen ciphertext (CCA2) security and the homomorphic property can never be achieved simultaneously. In other words, security is sacrificed in exchange for the homomorphic property. Although several previous works (e.g., [2, 9, 10, 25, $42,43]$ ) have attempted to construct homomorphic PKE schemes that offer security close to CCA2 security while retaining the homomorphic property, these schemes only guarantee security at limited levels. Note that not all functionalities of conventional homomorphic encryption are indispensable for real-world applications, and therefore there is the possibility of realizing a desirable security level by appropriately selecting the functionalities of conventional homomorphic encryption.

Here, we point out that the underlying cause of the incompatibility of CCA2 security and the homomorphic property, lies in the setting that any user can use the homomorphic property, and it is worth discussing whether the free availability of homomorphic operations is an indispensable functionality in real-world applications. For example, consider the situation where some data encrypted by a homomorphic PKE scheme is stored in a public database (e.g., public cloud computing environment) and it is modified by homomorphic operations. If anyone can perform a homomorphic operation, then it is hard to reduce the risk of unexpected changes to the encrypted data in the database in which resources are dynamically allocated. Even in a closed environment (e.g., private cloud computing environment), we cannot rule out the possibility of unexpected changes to a user's data by any user who is authorized to access the database. Of course, it is possible to protect such unexpected modification of encrypted data by setting access permissions of each user appropriately. However, in cloud environments, security of outsourced data storages may not be assured. Therefore, such access control functionality should be included in encrypted data itself.

From the above consideration, we see that the property that anyone can perform homomorphic operations not only inhibits the realization of CCA2 security, but also introduces the problem of unexpected modification of encrypted data.

\subsection{Our Contribution}

In this paper, we show that CCA2 security and the homomorphic property can be simultaneously handled in situations that the user(s) who can perform homomorphic operations should be controlled. Specifically, we propose a new concept of homomorphic PKE, which we call keyed-homomorphic public-key encryption (KH-PKE), that has the following properties: (1) in addition to a conventional public/decryption key pair $\left(p k, s k_{d}\right)$, another secret key for the homomorphic operation (denoted by $s k_{h}$ ) is introduced, $(2)$ homomorphic operations cannot be performed without using $s k_{h}$, and (3) ciphertexts cannot be decrypted using only $s k_{h}$. Interestingly, KH-PKE implies conventional homomorphic PKE, since the latter can be implemented by publishing $s k_{h}$ of KH-PKE.

Here we introduce situations that the user(s) who can perform homomorphic operations should be controlled/limited. For example, in the situation where encrypted data is stored in a public database, an owner of the data gives $s k_{h}$ to the database manager, who updates the encrypted data after authentication of users. No outsider can modify the encrypted data in the public database without having $s k_{h}$. As another example, by considering $s k_{h}$, a counter can take over the role of aggregating an audience survey, voting, and so on. An advantage of separating ballot-counting and ballot-aggregation is that it is possible to reduce the aggregation costs of the counter and to collect the ballot results for individual areas, groups, and communities. We can also consider an application of KH-PKE to prevent illegal distribution of data. A content creator gives $s k_{h}$ to a digital content provider and the provider embeds some data (e.g., a water mark) for protecting the content against illegal copying, a certification for ownership of the content, and/or a distribution route.

To construct KH-PKE schemes, we introduce a new concept, transitional universal property, which can be obtained from any diverse group system [15], and present a number of KH-PKE schemes through hash proof systems (HPSs) [15]. As concrete instantiations, we present a practical KH-PKE scheme with multiplicative homomorphic operations from the decisional Diffie-Hellman (DDH) assumption and also present a practical 
KH-PKE scheme with additive homomorphic operations from the decisional composite residuosity (DCR) assumption, respectively. We remark that other (multiplicative) KH-PKE schemes based on the decisional linear (DLIN) assumption and the decisional bilinear Diffie-Hellman (DBDH) assumption can be constructed from the Shacham HPS [44] and the Galindo-Villar HPS [21], respectively. Moreover, we also consider an identity-based analogue of KH-PKE, called keyed-homomorphic identity-based encryption (KH-IBE) and give its concrete construction from the Gentry IBE scheme [22].

Naive Construction and its Limitations: One might think that the functionality and the security of KH-PKE can be achieved by using the following double encryption methodology: A ciphertext of an "inner" CCA1 secure homomorphic PKE scheme is encrypted by an "outer" CCA2 secure PKE scheme, and the decryption key of the CCA2 secure PKE scheme is used as $s k_{h}$. However, this naive construction is not secure in the sense of our security definition. Taking into account the exposure of the homomorphic operation key $s k_{h}$, an adversary can request $s k_{h}$ to be exposed in our security definition. The adversary is allowed to use the decryption oracle "even after the challenge phase", just before the adversary requests $s k_{h}$. However, no such decryption query is allowed in the CCA1 security of the underlying "inner" scheme, and therefore it seems hard to avoid this problem.

Even if we turn a blind eye to the above problem, it is obvious that efficiency of the naive construction is roughly equal to the total costs of the building block PKE schemes. On the other hand, the efficiency of our KH-PKE instantiations is very close to the corresponding (non-keyed-homomorphic) PKE schemes based on HPSs. In particular, the efficiency of our DDH-based KH-PKE scheme is comparably efficient as the Cramer-Shoup PKE (CS) scheme [13], where for $\ell$-bit security, our scheme yields only $\ell$-bit longer ciphertext size than that of the CS PKE scheme. Whereas the naive construction yields at least $5 \ell$-bit longer ciphertext size even if we choose the Kurosawa-Desmedt (KD) PKE scheme [35] and the CramerShoup lite PKE scheme [13] that seems the most efficient combination under the DDH assumption. We give the comparison in Table 7 in Section 5.3.

To sum up, our construction is superior to the naive construction from both security and efficiency perspectives.

Our Methodology: As a well-known result, CCA2-secure PKE can be constructed via a HPS [15] which has two projective hash families as its internal structure: A universal l $_{2}$ projective hash and a smooth projective hash. Also it is known that a weaker property of universal ${ }_{2}$, that is called universal $l_{1}$ property, was shown to be useful for achieving CCA1-secure PKE [34], and universal 1 property (and smooth property also) does not contradict the homomorphic property. That is, our aim seems to be achieved if we can establish a switching mechanism from universal ${ }_{2}$ to universal $_{1}$. Moreover, we can simulate the decryption oracle even after the challenge phase and after revealing $s k_{h}$ since the simulator knows all secret keys in the security proof.

In this paper, we show such a mechanism, which we call transitional universal property, can be obtained from any diverse group system [15], then we propose a generic construction of KH-PKE through HPSs. Moreover, as an implication result, KH-PKE is implied by CPA-secure homomorphic PKE (with cyclicgroup ciphertext space) which implies diverse group systems [27].

Other KH-PKEs and Towards Constructing Keyed-FHE from Simple Assumptions: Here, we summarize the current known KH-PKE schemes. See Table 1. Following our work, Libert, Peters, Joye, and Yung (LPYJ) [38] proposed a KH-PKE scheme for supporting threshold decryption and publicly verifiable ciphertexts. They apply linearly homomorphic structure-preserving signatures [37] to quasi-adaptive noninteractive zero-knowledge (QA-NIZK) proofs [29], proposed QA-NIZK proofs with unbounded simulationsoundness (USS), and constructed a KH-PKE scheme by applying USS. The LPYJ KH-PKE scheme (with multiplicative homomorphic operations) is secure under the DLIN assumption (and strong unforgeability of the underlying one-time signature). Jutla and Roy (JR) [30] also proposed a publicly verifiable KH-PKE scheme with shorter ciphertext size, where a ciphertext of the LPJY scheme consists 9 group elements and two more in the other plus a one-time signature key pair and that of the JR scheme is 6 group elements. Their scheme relies on the symmetric external Diffie-Hellman (SXDH) assumption. We remark that our 
Table 1: KH-PKE schemes

\begin{tabular}{|c||c|c|}
\hline & Homomorphism & Complexity Assumptions \\
\hline \hline LPJY [38] & Multiplicative & DLIN \\
\hline JR [30] & Multiplicative & SXDH \\
\hline LDMSW [36] & Full & LWE \& $i \mathcal{O}$ \\
\hline Ours 1 & Multiplicative & DDH \\
\hline Ours 2 & Additive & DCR \\
\hline
\end{tabular}

KH-PKE schemes have a shorter ciphertext size than those of the LPYJ/JR schemes though our schemes do not support public verifiability. Moreover, our schemes are pairing-free whereas the LPYJ/JR schemes require bilinear groups.

Though these KH-PKE schemes including ours support either additive or multiplicative homomorphic operation, Lai, Deng, Ma, Sakurai, and Weng (LDMSW) [36] proposed Keyed-Fully Homomorphic Encryption (Keyed-FHE) which supports the evaluation of any functions on encrypted data. They first constructed convertible identity-based fully homomorphic encryption (IBFHE) from the learning with errors (LWE) assumption, and proposed a generic conversion of Keyed-FHE from IBFHE. One drawback of the Lai et al. scheme is to employ indistinguishability obfuscation $(i \mathcal{O})$ [3] which is recognized as one of the extremely strong cryptographic tools. Since we give a generic construction of KH-PKE from HPSs, one may expect that KH-PKE from lattices can be constructed via HPSs from lattices, e.g., [31]. However, to the best of our knowledge, universal ${ }_{2}$ projective hash from lattices, which is a main property we require, has not been proposed so far. Moreover, we additionally require transitional universal property of the pair of two HPSs. Constructing such a universal ${ }_{2}$ projective hash from lattices lead to construct KH-PKE from lattices and may also lead to construct Keyed-FHE from simple assumptions. We leave it as a future work of this paper.

\subsection{Related Work}

Several previous works have attempted to construct homomorphic PKE schemes that provide security close to CCA2 security, while retaining the homomorphic property. Canetti et al. [10] proposed CCA1-secure FHE schemes from multi-key identity-based FHE. Canetti et al. [9] considered the notion of replayable CCA (RCCA), which leaves a room for an adversary who is given two ciphertexts $\left(C, C^{\prime}\right)$, to gain information on whether $C^{\prime}$ was derived from $C$. (Modified RCCA notions have also been proposed [25, 42].) In the RCCA security game, the decryption oracle given to an adversary is restricted in such a way that the challenge ciphertext and ciphertexts derived from the challenge ciphertext cannot be queried to the oracle. Similarly, in benignly-malleable (gCCA) security [2, 45], ciphertexts related to the challenge one cannot be input to the decryption oracle. Therefore, RCCA and gCCA are strictly weaker notions than CCA2, and may not be sufficient if the encryption scheme is used as a building block for higher level protocols/systems.

In [43], Prabhakaran and Rosulek proposed homomorphic CCA (HCCA) security, where only the expected operation, and no other operations, can be performed for any ciphertext. (Targeted malleability, which is a similar concept to HCCA, was considered in [7].) In addition, they also showed that CCA2, gCCA, and RCCA are special cases of HCCA. Note that HCCA does not handle the homomorphic property and CCA2 security simultaneously, since anyone can perform the homomorphic operation. Chase et al. [12] showed that controlled-malleable non-interactive zero-knowledge can be used as a general tool for achieving RCCA and HCCA security.

Embedding a ciphertext of homomorphic PKE into that of CCA2-secure PKE, was considered in [39, 6]. Note that their embedding encryption methods are nothing more than protecting a ciphertext of homomorphic PKE by that of CCA2 PKE, and therefore no homomorphic operation can be performed on embedded ciphertexts. Meanwhile, in our KH-PKE, even after performing the homomorphic operation, a ciphertext is still valid. 
Barbosa and Farshim [4] proposed delegatable homomorphic encryption (DHE). The difference between KH-PKE and DHE is that in DHE a trusted authority (TA) issues a token to control the capability to evaluate circuits $f$ over encrypted data $M$ to untrusted evaluators. Furthermore, their security definitions of DHE (input/output privacy (TA-IND-CPA) and evaluation security (IND-EVAL2)) do not allow an adversary to access the decryption oracle and the evaluation oracle (the oracle for homomorphic operation) simultaneously. We note that although Barbosa and Farshim defined verifiability (VRF-CCA2), where no homomorphic operation can be performed without issuing a corresponding token, KH-CCA security for KH-PKE defined in this paper guarantees a similar level of security, since if there exists an adversary that can perform the homomorphic operation without using $s k_{h}$, then the adversary can break the KH-CCA security.

Desmedt, Iovino, Persiano, and Visconti [17] proposed a controllable homomorphic encryption scheme (CHES) where a ciphertext is allowed to be evaluated only if the corresponding token is given. This tokenoriented homomorphic property is the same as that of our keyed-homomorphic property. As a difference, they just considered CPA security and did not consider CCA security against an adversary who does not have the corresponding tokens.

As an application of KH-IBE, Emura et al. [20] proposed mis-operation resistant homomorphic encryption where a keyword and data are encrypted simultaneously, and the evaluation algorithm posts alert to homomorphic operations for ciphertexts associated with different keywords. Moreover, even if one intentionally or accidentally performs the homomorphic operations against such ciphertexts, the decryption algorithm rejects such artificially-mixed ciphertexts, and the receiver can recognize a mis-operation happens in the evaluation phase.

In the signature context, Abe et al. [1] considered selective randomizability, where a strongly unforgeable signature to be randomized with the help of a randomization token, and a randomizable signature is still existentially unforgeable.

\subsection{Differences from the Proceedings Version [19]}

In the proceedings version [19], there were several bugs. Specifically, in the second last component $\widehat{\pi}$ of a ciphertext of the generic construction (in [19, Fig. 1]) and that of the DDH-based construction (in [19, Fig. 2]) could be malleable, which could lead to CCA attacks on the schemes. Furthermore, the evaluation algorithms for these constructions were (although "correct" in terms of the functionality of KH-PKE) not properly designed in the sense that in the CCA security game, the result of the "evaluation oracle" for challenge ciphertext-dependent inputs could leak some information. Moreover, we did not properly state the requirement of the second hash function (denoted by $\mathrm{TCR}_{2}$ in [19, Fig. 2]) used to "compress" the proof value $\widetilde{\pi}$ to reduce the ciphertext size.

We fix these bugs in the current version: First, we reconsider the proposed generic construction (in Section 4): (1) the first proof value $\widetilde{\pi}$ in the generic construction (in Fig. 1) is now made dependent on the second proof value $\widehat{\pi}$, and (2) the evaluation algorithm Eval computes and "adds" a new ciphertext of 0 . These modifications enable us to prove our modified proposed constructions to be CCA secure. According to these modifications, we do not have to newly define homomorphic transitional universal hash family. Instead, we introduce transitional universal property of the pair of two HPSs. We also apply the similar modifications to our DDH-based scheme (in Section 5.3). We would like to emphasize that the modifications here do not incur additional computational cost or increase of the ciphertext size for both of our constructions.

Second, we reconsider the condition of the function that is used to "compress" the proof value $\widetilde{\pi}$ in our DDH-based construction, and newly introduce the notion of smoothness for a function. This is a statistical property that roughly ensures that the "min-entropy" of the output of a function (for uniformly random input) is sufficiently high, and thus it is information-theoretically hard to guess the output of a function for random inputs. We also show that natural cryptographic functions, a one-way function (OWF), an always second-preimage resistant (aSec secure) hash function [41], and a key derivation function (KDF) [16], have the property, and thus in practice we can use (an appropriate modification of) cryptographic hash functions such as SHA-series).

Third, we give a formal definition of KH-IBE and its concrete construction from the Gentry IBE scheme $[22]$ in this version. 


\subsection{Organization}

This paper is organized as follows. We review the basic notations and definitions related to HPSs in Section 2. We give the syntax and security definition of KH-PKE in Section 3. We give our generic construction of KH-PKE from HPSs and its security proofs in Section 4.2 and Section 4.3 respectively. We instantiate our generic construction from the DDH and DCR assumptions in Section 5.3 and Section 5.4 respectively. Finally, we introduce KH-IBE in Section 6.

\section{Preliminaries}

In this section, we review the basic notations and definitions related to HPSs (mostly following [15] but slightly customized for our convenience).

Throughout this paper, PPT denotes probabilistic polynomial time. If $n$ is a natural number, then $[n]=\{1, \ldots, n\}$. If $D$ is a probability distribution (over some set), then $[D]$ denotes its support, i.e. $[D]=\left\{x \mid \operatorname{Pr}_{x^{\prime} \leftarrow D}\left[x^{\prime}=x\right]>0\right\}$. Let $\mathbf{X}=\left\{X_{\ell}\right\}_{\ell \geq 0}$ and $\mathbf{Y}=\left\{Y_{\ell}\right\}_{\ell \geq 0}$ be sequences of random variables $X_{\ell}$ and $Y_{\ell}$, respectively, defined over a same finite set. As usual, we say that $\mathbf{X}$ and $\mathbf{Y}$ are statistically (resp. computationally) indistinguishable if $\left|\operatorname{Pr}\left[\mathcal{A}\left(X_{\ell}\right)=1\right]-\operatorname{Pr}\left[\mathcal{A}\left(Y_{\ell}\right)=1\right]\right|$ is negligible in $\ell$ for any computationally unbounded (resp. PPT) algorithm $\mathcal{A}$. Furthermore, we say that $\mathbf{X}$ and $\mathbf{Y}$ are $\epsilon$-close if the statistical distance of $X_{\ell}$ and $Y_{\ell}$ is at most $\epsilon=\epsilon(\ell)$. For a finite set $B_{\ell}$ and its subset $B_{\ell}^{\prime}$ indexed (often implicitly) by $\ell \geq 0$, we say that $B_{\ell}^{\prime}$ is approximately samplable relative to $B_{\ell}$, if there is a sequence of random variables on $B_{\ell}$ which is polynomial-time samplable and is statistically indistinguishable from the uniform random variable on $B_{\ell}^{\prime}$.

Projective Hash Families: Let $X, \Pi, K$, and $S$ be finite non-empty sets, $X^{\prime}$ be a non-empty subset of $X$, and $L$ be a proper subset of $X$ (i.e., $L \subset X$ and $L \neq X)$. Furthermore, let $H=\left\{H_{k}: X \rightarrow \Pi\right\}_{k \in K}$ be a collection of hash functions indexed by $k \in K$, and $\alpha: K \rightarrow S$ be a function. We say that $\mathbf{H}=$ $\left(H, K, X, X^{\prime}, L, \Pi, S, \alpha\right)$ is a projective hash family for $\left(X, X^{\prime}, L\right)$, if for all $k \in K$, the action of $H_{k}$ on the subset $L$ is uniquely determined by $\alpha(k) \in S$. When $X^{\prime}=X$, we may omit the symbol $X^{\prime}$ in the notations above.

Let $\mathbf{H}=\left(H, K, X, X^{\prime}, L, \Pi, S, \alpha\right)$ be a projective hash family, and let $0 \leq \epsilon \leq 1$. We recall the following

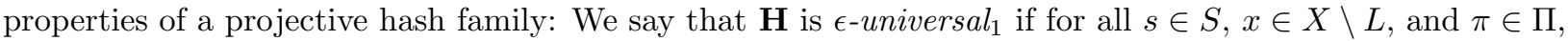
it holds that

$$
\operatorname{Pr}_{k \stackrel{\Phi}{\leftarrow} K}\left[H_{k}(x)=\pi \wedge \alpha(k)=s\right] \leq \epsilon \cdot \underset{k \stackrel{\$}{\leftarrow} K}{\operatorname{Pr}}[\alpha(k)=s] .
$$

We say that $\mathbf{H}$ is $\epsilon$-universal 2 if for all $s \in S, x, x^{*} \in X \backslash L$ with $x^{*} \neq x$, and $\pi, \pi^{*} \in \Pi$, it holds that

$$
\operatorname{Pr}_{k \stackrel{\$}{\leftarrow} K}\left[H_{k}(x)=\pi \wedge H_{k}\left(x^{*}\right)=\pi^{*} \wedge \alpha(k)=s\right] \leq \epsilon \cdot \underset{k \leftarrow K}{\operatorname{Pr}}\left[H_{k}\left(x^{*}\right)=\pi^{*} \wedge \alpha(k)=s\right] .
$$

We say that $\mathbf{H}$ is $\epsilon$-smooth if the following two distributions are $\epsilon$-close:

$$
\begin{gathered}
\left\{k \stackrel{\$}{\leftarrow} K ; x \stackrel{\$}{\leftarrow} X \backslash L:\left(\alpha(k), x, H_{k}(x)\right)\right\} \\
\text { and } \\
\{k \stackrel{\$}{\leftarrow} K ; x \stackrel{\$}{\leftarrow} X \backslash L ; \pi \stackrel{\$}{\leftarrow} \Pi:(\alpha(k), x, \pi)\} .
\end{gathered}
$$

We also introduce a variant of the smoothness property: Suppose that $\Pi$ is an abelian group (written in additive form), and let $\Pi^{\prime}$ be a subgroup of $\Pi$. In this case, we say that $\mathbf{H}$ is $\epsilon$-smooth relative to $\left(X^{\prime}, \Pi^{\prime}\right)$, if the following two distributions are $\epsilon$-close: 


$$
\begin{gathered}
\left\{k \stackrel{\$}{\leftarrow} K ; x \stackrel{\$}{\leftarrow} X^{\prime} \backslash L:\left(\alpha(k), x, H_{k}(x)\right)\right\} \\
\text { and } \\
\left\{k \stackrel{\$}{\leftarrow} K ; x \stackrel{\$}{\leftarrow} X^{\prime} \backslash L ; \pi \stackrel{\$}{\leftarrow} \Pi^{\prime}:\left(\alpha(k), x, H_{k}(x)+\pi\right)\right\} .
\end{gathered}
$$

We note that, when $\Pi^{\prime}=\Pi$, the term $H_{k}(x)+\pi$ in the latter probability distribution above can be replaced with $\pi$, since now $H_{k}(x)+\pi$ is also uniformly random over $\Pi$. Hence, the notion of smoothness relative to $\left(X^{\prime}, \Pi^{\prime}\right)$ above is in fact a generalization of the smoothness.

If a projective hash family is $\epsilon$-universal ${ }_{1}$ (resp. - universal ${ }_{2}$,-smooth) for a negligible $\epsilon$, then we simply call the projective hash family universal ${ }_{1}$ (resp. universal ${ }_{2}$, smooth). We note that the $\epsilon$-universal ${ }_{2}$ property implies the $\epsilon$-universal ${ }_{1}$ property, by summing up the inequalities in the definition of the universal ${ }_{2}$ property over all $\pi^{*} \in \Pi$. We also show some relations between the smoothness property and the universal ${ }_{1}$ property.

Lemma 2.1. If a projective hash family $\mathbf{H}=(H, K, X, L, \Pi, S, \alpha)$ is 0 -smooth, then it is $(1 /|\Pi|)$-universal . $_{1}$

Proof. Since $\mathbf{H}$ is 0-smooth, the two distributions appearing in the definition of the smoothness for $\mathbf{H}$ are identical. Therefore, for any $x \in X \backslash L, s \in S$ and $\pi \in \Pi$, we have

$$
\begin{aligned}
& \operatorname{Pr}_{k \stackrel{\&}{\leftarrow} K}\left[\left(\alpha(k), H_{k}(x)\right)=(s, \pi)\right]
\end{aligned}
$$

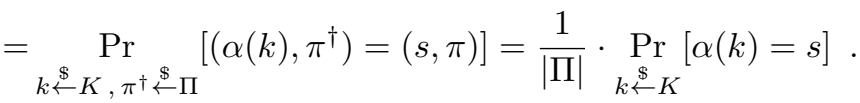

This implies that $\mathbf{H}$ is $(1 /|\Pi|)$-universal ${ }_{1}$, as desired.

Lemma 2.2. If a projective hash family $\mathbf{H}=(H, K, X, L, \Pi, S, \alpha)$ is $\epsilon$-universal ${ }_{1}$, then it is $\epsilon^{\prime}$-smooth where $\epsilon^{\prime}=(\epsilon|\Pi|-1)(|\Pi|-1) / 2$. In particular, if a projective hash family $\mathbf{H}=(H, K, X, L, \Pi, S, \alpha)$ is $(1 /|\Pi|)$ universal 1 , then it is 0 -smooth.

Proof. First, we note that $\epsilon \geq 1 /|\Pi|$ by the definition of the $\epsilon$-universal ${ }_{1}$ property. Since $\mathbf{H}$ is $\epsilon$-universal ${ }_{1}$, for any $x \in X \backslash L, s \in S$ and $\pi \in \Pi$, we have

$$
\begin{aligned}
& \operatorname{Pr}_{k \leftarrow K, x^{\dagger} \leftarrow-\mathbb{\&} \backslash L}\left[\left(\alpha(k), x^{\dagger}, H_{k}\left(x^{\dagger}\right)\right)=(s, x, \pi)\right]=\frac{1}{|X \backslash L|} \cdot \operatorname{Pr}_{k \leftarrow K}\left[\left(\alpha(k), H_{k}(x)\right)=(s, \pi)\right]
\end{aligned}
$$

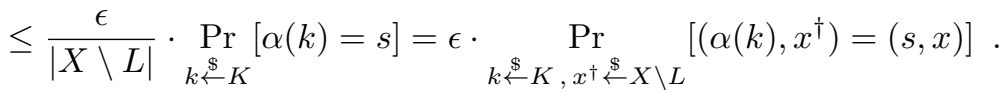

Since the right-hand side is independent of $\pi$, by summing up the inequality over all $\pi \in \Pi$ except a fixed $\pi^{\prime} \in \Pi$, we have

$$
\operatorname{Pr}_{k \stackrel{\&}{\leftarrow} K, x^{\dagger} \stackrel{⿱}{\leftarrow} X \backslash L}\left[\left(\alpha(k), x^{\dagger}\right)=(s, x) \wedge H_{k}\left(x^{\dagger}\right) \neq \pi^{\prime}\right] \leq(|\Pi|-1) \epsilon \cdot \operatorname{Pr}_{k \leftarrow K, x^{\dagger} \stackrel{\mathbb{E}}{\leftarrow} X \backslash L}\left[\left(\alpha(k), x^{\dagger}\right)=(s, x)\right],
$$

therefore

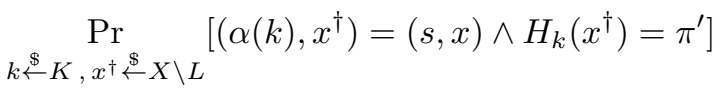

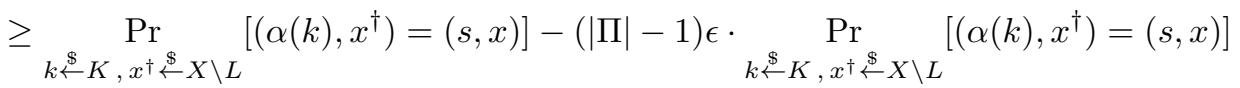

$$
\begin{aligned}
& =(1-(|\Pi|-1) \epsilon) \cdot \operatorname{Pr}_{k \leftarrow-\S} \operatorname{Pr}_{\leftarrow \dagger}\left[\left(\alpha(k), x^{\dagger}\right)=(s, x)\right] .
\end{aligned}
$$


By combining this inequality (where $\pi^{\prime}$ is replaced with $\pi$ ) with the first inequality above, and by using the

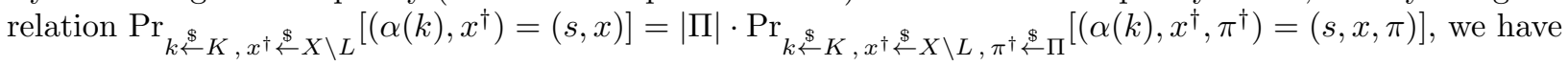

$$
\begin{aligned}
& (|\Pi|-(|\Pi|-1)|\Pi| \epsilon) \cdot \underset{k \stackrel{\$}{\leftarrow} K, x^{\dagger} \stackrel{\$}{\leftarrow} X \backslash L, \pi^{\dagger} \stackrel{\mathbb{\&}}{\leftarrow} \Pi}{\operatorname{Pr}}\left[\left(\alpha(k), x^{\dagger}, \pi^{\dagger}\right)=(s, x, \pi)\right]
\end{aligned}
$$

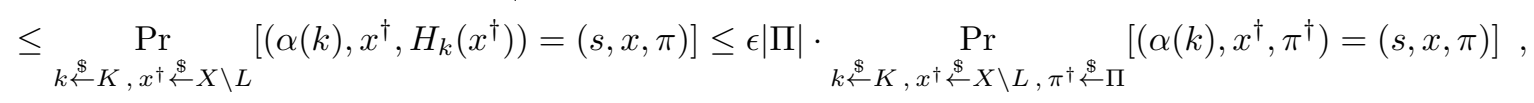

therefore $($ since $\epsilon \geq 1 /|\Pi|)$

$$
\begin{aligned}
& \left|\operatorname{Pr}_{k \stackrel{\$}{\leftarrow} K, x^{\dagger} \stackrel{\$}{\leftarrow} X \backslash L}\left[\left(\alpha(k), x^{\dagger}, H_{k}\left(x^{\dagger}\right)\right)=(s, x, \pi)\right]-\underset{k \stackrel{\$}{\leftarrow} K, x^{\dagger} \stackrel{\operatorname{\$ r}}{\leftarrow} X \backslash L, \pi^{\dagger} \stackrel{\$}{\leftarrow} \Pi}{\operatorname{Pr}}\left[\left(\alpha(k), x^{\dagger}, \pi^{\dagger}\right)=(s, x, \pi)\right]\right|
\end{aligned}
$$

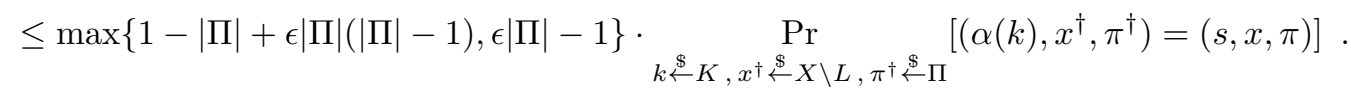

By summing up the inequality over all $s \in S, x \in X \backslash L$ and $\pi \in \Pi$, and by dividing it by two, the statistical distance between the two distributions appearing in the definition of the smoothness for $\mathbf{H}$ is bounded by $\max \{1-|\Pi|+\epsilon|\Pi|(|\Pi|-1), \epsilon|\Pi|-1\} / 2$. Note that $1-|\Pi|+\epsilon|\Pi|(|\Pi|-1)=(\epsilon|\Pi|-1)(|\Pi|-1)$. Now if $|\Pi|=1$, then we have $\epsilon=1$ since $1 /|\Pi| \leq \epsilon \leq 1$, therefore $(\epsilon|\Pi|-1)(|\Pi|-1)=\epsilon|\Pi|-1=0$. On the other hand, if $|\Pi| \geq 2$, then we have $(\epsilon|\Pi|-1)(|\Pi|-1) \geq \epsilon|\Pi|-1$, therefore $\max \{1-|\Pi|+\epsilon|\Pi|(|\Pi|-1), \epsilon|\Pi|-1\} / 2=$ $(\epsilon|\Pi|-1)(|\Pi|-1) / 2$. Hence, the claim holds.

Subset Membership Problems: A subset membership problem M specifies a collection of probabilistic distribution $\left\{I_{\ell}\right\}_{\ell \geq 0}$ (indexed by a security parameter $\ell$ ) over instance descriptions. An instance description $\Lambda\left[X, X^{\prime}, L, W, R\right] \in\left[I_{\ell}\right]$ specifies a non-empty set $X$ and its non-empty subsets $X^{\prime}, L \subset X$, a non-empty set $W$, and a binary relation $R$ defined over $X \times W$ with the property that an $x \in X$ is in the subset $L$ if and only if there exists a "witness" $\omega \in W$ such that $(x, w) \in R$. When $X^{\prime}=X$, we may simply write $\Lambda[X, L, W, R]$ instead of $\Lambda\left[X, X^{\prime}, L, W, R\right]$. Moreover, if these objects are clear from the context, we will just write $\Lambda$ to indicate an instance description.

We require that a subset membership problem $\mathbf{M}$ provides the following algorithms: (1) the instance sampling algorithm takes as input $1^{\ell}$, and returns $\Lambda\left[X, X^{\prime}, L, W, R\right] \in\left[I_{\ell}\right]$ chosen according to $I_{\ell}$, and (2) the subset sampling algorithm takes as input $1^{\ell}$ and an instance $\Lambda\left[X, X^{\prime}, L, W, R\right] \in\left[I_{\ell}\right]$, and returns $x \stackrel{\$}{\leftarrow} L$ and a witness $\omega \in W$ for $x$. We say that a subset membership problem $\mathbf{M}=\left\{I_{\ell}\right\}_{\ell \geq 0}$ is hard relative to $X^{\prime}$, if the following two distributions are computationally indistinguishable:

$$
\left\{\Lambda \leftarrow I_{\ell} ; x \stackrel{\$}{\leftarrow} L:(\Lambda, x)\right\} \text { and }\left\{\Lambda \leftarrow I_{\ell} ; x \stackrel{\$}{\leftarrow} X^{\prime} \backslash L:(\Lambda, x)\right\}
$$

When $X^{\prime}=X$, we simply say that $\mathbf{M}$ is hard.

Hash Proof System (HPS): Informally, a HPS is a special kind of (designated-verifier) non-interactive zero-knowledge proof system for a subset membership problem $\mathbf{M}=\left\{I_{\ell}\right\}_{\ell>0}$. A HPS has, as its internal structure, a family of hash functions with the special projective property, and this projective hash family is associated with each instance of the subset membership problems. Although HPS does not treat for all NP languages, HPS leads to an efficient CCA2-secure PKE construction.

As in [15], we will occasionally introduce an arbitrary finite set $E$ to extend the sets $X, X^{\prime}$ and $L$ in an instance $\Lambda\left[X, X^{\prime}, L, W, R\right] \in\left[I_{\ell}\right]$ of $\mathbf{M}$ into $X \times E, X^{\prime} \times E$ and $L \times E$. If $E$ is not required (e.g., for a smooth HPS in our construction of KH-PKE), then we omit $E$ from the following algorithms. A HPS $\mathbf{P}=$ (HPS.param, HPS.priv, HPS.pub), for $\mathbf{M}$ associates each instance $\Lambda=\Lambda\left[X, X^{\prime}, L, W, R\right]$ of $\mathbf{M}$ with a projective hash family $\mathbf{H}=\left(H, K, X \times E, X^{\prime} \times E, L \times E, \Pi, S, \alpha\right)$, provides the following three efficient algorithms: 
1. The index sampling algorithm HPS.param takes an instance $\Lambda$ as input, and returns $k \in K$ and $s \in S$ such that $\alpha(k)=s$.

2. The private evaluation algorithm HPS.priv takes $\Lambda \in\left[I_{\ell}\right], k \in K$ and $(x, e) \in X \times E$ as input, and returns $\pi=H_{k}(x, e) \in \Pi$.

3. The public evaluation algorithm HPS.pub takes $\Lambda \in\left[I_{\ell}\right], s \in S, x \in L, e \in E$, and a witness $\omega$ for $x$ as input, and returns $\pi=H_{k}(x, e) \in \Pi$.

We say that $\mathbf{P}$ is $\epsilon$-universal ${ }_{1}$ (resp. $\epsilon$-universal ${ }_{2}, \epsilon$-smooth) if for all $\ell>0$ and for all $\Lambda \in\left[I_{\ell}\right]$, $\mathbf{H}$ is an $\epsilon$-universal ${ }_{1}$ (resp. $\epsilon$-universal $2, \epsilon$-smooth) projective hash family.

The following homomorphic property of hash proof systems is required in our proposed construction.

Definition 2.1 (Homomorphic Projective Hash Family). We say that a projective hash family $\mathbf{H}=(H, K, X \times$ $\left.E, X^{\prime} \times E, L \times E, \Pi, S, \alpha\right)$ is homomorphic, if $X, E$ and $\Pi$ are abelian groups (written in additive form), $L$ is a subgroup of $X$, and we have $H_{k}\left(x_{1}\right)+H_{k}\left(x_{2}\right)=H_{k}\left(x_{1}+x_{2}\right)$ for any $k \in K$ and $x_{1}, x_{2} \in X$. We also say that a hash proof system $\mathbf{P}$ is homomorphic, if the underlying projective hash family is homomorphic. (We note that $X^{\prime}$ is not required to be a subgroup of $X$.)

Diverse Group System and Derived Projective Hash Family: Here, we recall the definition of diverse group systems introduced in [15], which were used to construct projective hash families. Let $X$, $L$, and $\Pi$ be abelian groups, where $L$ is a proper subgroup of $X$, and $\operatorname{Hom}(X, \Pi)$ be the group of all homomorphisms $\phi: X \rightarrow \Pi$. Let $\mathcal{H}$ be a subgroup of $\operatorname{Hom}(X, \Pi)$. Then $\mathbf{G}=(\mathcal{H}, X, L, \Pi)$ is called a group system. In addition, we say that $\mathbf{G}$ is diverse if for all $x \in X \backslash L$, there exists $\phi \in \mathcal{H}$ such that $\phi(L)=\langle 0\rangle$, but $\phi(x) \neq 0$.

We recall the projective hash family $\mathbf{H}=(H, K, X, L, \Pi, S, \alpha)$ derived from a diverse group system $\mathbf{G}$ ([15, Definition 2]): The instance $\Lambda=\Lambda[X, L, W, R]$ of the underlying subset membership problem satisfies that $W=\left(\mathbb{Z}_{|L|}\right)^{d}$ and $\left(x,\left(\omega_{1}, \ldots, \omega_{d}\right)\right) \in R$ if and only if $x=\sum_{i=1}^{d} \omega_{i} g_{i}$, where $\left\{g_{1}, \ldots, g_{d}\right\}$ is a fixed generating set of $L$. Let the elements of the subgroup $\mathcal{H}$ of $\operatorname{Hom}(X, \Pi)$ be indexed as $\mathcal{H}=\left\{H_{k} \mid k \in K\right\}$ for a set $K$. Set $S=\Pi^{d}$, and define $\alpha: K \rightarrow S$ by $\alpha(k)=\left(\phi\left(g_{1}\right), \ldots, \phi\left(g_{d}\right)\right)$, where $\phi=H_{k}$. Note that $\mathbf{H}$ is a homomorphic projective hash family because $H_{k}(x)$ for $x \in L$ is determined by $\alpha(k)$ such that $H_{k}(x)=\phi\left(\sum_{i=1}^{d} \omega_{i} g_{i}\right)=\sum_{i=1}^{d} \omega_{i} \phi\left(g_{i}\right)$. The following was shown by Cramer and Shoup [15, Theorem 2].

Lemma 2.3. The projective hash family $\mathbf{H}$ derived from a diverse group system $\mathbf{G}$ as above is $1 / \widetilde{p}$-universal ${ }_{1}$, where $\widetilde{p}$ is the smallest prime dividing $|X / L|$.

\section{Definition of KH-PKE}

In this section, we give the formal definitions of the syntax and the security requirements of KH-PKE.

\subsection{Syntax of KH-PKE}

Definition 3.1 (Syntax of KH-PKE for homomorphic operation $\odot$ ). Let $\mathcal{M}$ be a message space and $\odot$ be a binary operation over $\mathcal{M}$. A KH-PKE scheme $\mathcal{K H}-\mathcal{P K \mathcal { E }}=($ KeyGen, Enc, Dec, Eval) for homomorphic operation $\odot$ consists of the following four algorithms:

KeyGen: This algorithm takes a security parameter $1^{\ell}(\ell \in \mathbb{N})$ as input, and returns a public key pk, a decryption key $s k_{d}$, and a homomorphic operation key $s k_{h}$.

Enc: This algorithm takes $p k$, and a message $M \in \mathcal{M}$ as input, and returns a ciphertext $C$.

Dec: This algorithm takes $s k_{d}$ and $C$ as input, and returns $M$ or $\perp$.

Eval: This algorithm takes $s k_{h}$, two ciphertexts $C_{1}$ and $C_{2}$ as input, and retunrs a ciphertext $C$ or $\perp$. 
Note that the above definition for the evaluation algorithm Eval does not say anything about the homomorphic property, and its functionality is defined as a correctness requirement below. Let $p k$ be a public key generated by the KeyGen algorithm, and $\mathcal{C}_{p k, M}$ be the set of all ciphertexts of $M \in \mathcal{M}$ under the public key $p k$, i.e., $\mathcal{C}_{p k, M}=\left\{C \mid \exists r \in\{0,1\}^{*}\right.$ s.t. $\left.C=\operatorname{Enc}(p k, M ; r)\right\}$.

Definition 3.2 (Correctness). A KH-PKE scheme for homomorphic operation $\odot$ is said to be correct if for all $\left(p k, s k_{d}, s k_{h}\right) \leftarrow \operatorname{KeyGen}\left(1^{\ell}\right)$, the following two conditions are satisfied: (1) For all $M \in \mathcal{M}$, and all $C \in \mathcal{C}_{p k, M}$, it holds that $\operatorname{Dec}\left(s k_{d}, C\right)=M$. (2) For all $M_{1}, M_{2} \in \mathcal{M}$, all $C_{1} \in \mathcal{C}_{p k, M_{1}}$, and all $C_{2} \in \mathcal{C}_{p k, M_{2}}$, it holds that $\operatorname{Eval}\left(s k_{h}, C_{1}, C_{2}\right) \in \mathcal{C}_{p k, M_{1} \odot M_{2}}$.

We call the Eval algorithm commutative if an operation $\odot$ is commutative, the distribution of $\operatorname{Eval}\left(s k_{h}, C_{1}\right.$, $\left.C_{2}\right)$ and that of $\operatorname{Eval}\left(s k_{h}, C_{2}, C_{1}\right)$ are identical. In fact, our KH-PKE schemes proposed in the paper are all commutative; for example, the homomorphic property of the DDH-based instantiation given in later section corresponds to the group operation in a multiplicative cyclic group.

Next, we define the security notion for KH-PKE, which we call indistinguishability of message under adaptive chosen ciphertext attacks (KH-CCA).

Definition 3.3 (KH-CCA). A KH-PKE scheme is said to be KH-CCA secure if for any PPT adversary $\mathcal{A}$, the advantage

$$
\begin{aligned}
& A d v_{\mathrm{KH}-\mathrm{PKE}, \mathcal{A}}^{K H-C C A}(\ell)=\mid \operatorname{Pr}\left[\left(p k, s k_{d}, s k_{h}\right) \leftarrow \operatorname{KeyGen}\left(1^{\ell}\right) ;\right. \\
& \left(M_{0}^{*}, M_{1}^{*}, \text { State }\right) \leftarrow \mathcal{A}^{\mathcal{O}}(\text { find }, p k) ; \beta \stackrel{\$}{\leftarrow}\{0,1\} ; \\
& \left.\left.\quad C^{*} \leftarrow \operatorname{Enc}\left(p k, M_{\beta}^{*}\right) ; \beta^{\prime} \leftarrow \mathcal{A}^{\mathcal{O}} \text { (guess, State, } C^{*}\right) ; \beta=\beta^{\prime}\right]-\frac{1}{2} \mid
\end{aligned}
$$

is negligible in $\ell$, where $\mathcal{O}$ consists of the three oracles $\operatorname{Eval}\left(s k_{h}, \cdot, \cdot\right)$, $\operatorname{RevHK}$, and $\operatorname{Dec}\left(s k_{d}, \cdot\right)$ defined as follows. Let $\mathcal{D}$ be a list which is set as $\mathcal{D}=\left\{C^{*}\right\}$ right after the challenge stage ( $\mathcal{D}$ is set as $\emptyset$ in the find stage).

- The evaluation oracle $\operatorname{Eval}\left(s k_{h}, \cdot, \cdot\right)$ : If RevHK has already been queried before, then this oracle is not available. Otherwise, this oracle responds to a query $\left(C_{1}, C_{2}\right)$ with the result of $C \leftarrow \operatorname{Eval}\left(s k_{h}, C_{1}, C_{2}\right)$. In addition, if $C \neq \perp$ and either $C_{1} \in \mathcal{D}$ or $C_{2} \in \mathcal{D}$, then the oracle updates the list by $\mathcal{D} \leftarrow \mathcal{D} \cup\{C\}$.

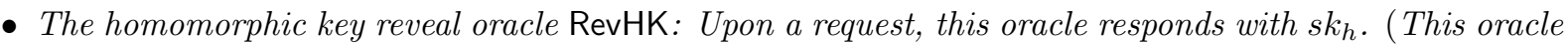
is available only once.)

- The decryption oracle $\operatorname{Dec}\left(s k_{d}, \cdot\right)$ : This oracle is not available if $\mathcal{A}$ has queried to $\operatorname{RevHK}$ and $\mathcal{A}$ has obtained the challenge ciphertext $C^{*}$. Otherwise, this oracle responds to a query $C$ with the result of $\operatorname{Dec}\left(s k_{d}, \cdot\right)$ if $C \notin \mathcal{D}$ or returns $\perp$ otherwise.

Here, let us remark on the definition of KH-CCA security. Throughout this paper, an adversary who has $s k_{h}$ is called an insider, whereas an adversary who does not have $s k_{h}$ is called an outsider.

In case $\mathcal{A}$ does not query the RevHK oracle (i.e., $\mathcal{A}$ is an outsider), $\mathcal{A}$ is allowed to adaptively issue decryption queries and evaluation queries of any ciphertexts. In particular, in order to capture the malleability in the presence of the homomorphic operation, the Eval oracle allows the challenge ciphertext $C^{*}$ as input. To avoid an unachievable security definition, the Dec oracle immediately answers $\perp$ for "unallowable ciphertexts" that are the results of a homomorphic operation for $C^{*}$ and any ciphertext of an adversary's choice. Such unallowable ciphertexts are maintained by the list $\mathcal{D}$.

The situation that the Dec oracle does not answer for ciphertexts that are derived from the challenge ciphertext $C^{*}$ might seem somewhat analogous to the definition of RCCA security [9]. However, there is a critical difference between KH-CCA and RCCA: In the RCCA security game, the Dec oracle does not answer if a ciphertext $C$ satisfies $\operatorname{Dec}\left(s k_{d}, C\right) \in\left\{M_{0}^{*}, M_{1}^{*}\right\}$. That is, the functionality of the Dec oracle is restricted regardless of the adversary's strategy. On the other hand, in the KH-CCA security game, in case 
an adversary selects the strategy that it does not submit $C^{*}$ to the Eval oracle, the restriction on the Dec oracle is exactly the same as the CCA2 security for ordinary PKE scheme, and it is one of the adversary's possible strategies whether it submits $C^{*}$ to the Eval oracle, and thus the adversary has more flexibility than in the RCCA game.

If an outsider $\mathcal{A}$ becomes an insider after $\mathcal{A}$ obtains the challenge ciphertext $C^{*}$, then $\mathcal{A}$ is not allowed to issue a decryption query after obtaining $s k_{h}$ via the RevHK oracle. In other words, $\mathcal{A}$ is allowed to issue a decryption query until right before obtaining $s k_{h}$, even if $C^{*}$ is given to $\mathcal{A}$. This restriction is again to avoid a triviality. (If $\mathcal{A}$ obtains $s k_{h}, \mathcal{A}$ can freely perform homomorphic operations over the challenge ciphertexts, and we cannot meaningfully define the "unallowable set" of ciphertexts.)

Note that we can show that any KH-CCA secure PKE scheme satisfies CCA1 (thus CPA also) security against an adversary who is given $\left(p k, s k_{h}\right)$ in the setup phase. Showing this implication is possible mainly due to the RevHK oracle that returns $s k_{h}$ to an adversary, and the Dec oracle in the KH-CCA game. Here, we explain how the implication of KH-CCA security to CCA1 security is proved. Let $\mathcal{A}$ be a CCA1 adversary. Using $\mathcal{A}$ as a building block, we can construct a reduction algorithm $\mathcal{B}$ that attacks KH-CCA security, as follows: First, $\mathcal{B}$ is firstly given $p k$. Then $\mathcal{B}$ asks the RevHK oracle to obtain $s k_{h}$, and runs $\mathcal{A}$ with input $\left(p k, s k_{h}\right)$. Wnen $\mathcal{A}$ sends a ciphertext $C$ as a decryption query, $\mathcal{B}$ forwards $C$ as $\mathcal{B}$ 's decryption query. After $\mathcal{A}$ submits $\left(M_{0}^{*}, M_{1}^{*}\right)$ as $\mathcal{A}$ 's challenge, $\mathcal{B}$ submits $\left(M_{0}^{*}, M_{1}^{*}\right)$ as $\mathcal{B}$ 's challenge. Given the challenge ciphertext $C^{*}, \mathcal{B}$ runs $\mathcal{A}$ with input $C^{*}$. When $\mathcal{A}$ terminates with output a guess bit, $\mathcal{B}$ uses what $\mathcal{A}$ outputs as its guess for the challenge bit, and terminates. It is easy to see that $\mathcal{B}$ perfectly simulates the CCA1 game for $\mathcal{A}$. Therefore, $\mathcal{B}$ 's KH-CCA advantage equals $\mathcal{A}$ 's CCA1 advantage. This implies that if the scheme is $\mathrm{KH}-\mathrm{CCA}$ secure, then the scheme is CCA1 secure as well.

\section{Generic Construction of KH-PKE}

In this section, we describe the proposed generic construction of KH-PKE scheme from projective hash families, and give the security proof. For the purpose, in Section 4.1, we introduce two "computationally secure" variants of the notion of universal ${ }_{2}$ projective hash families. Then in Section 4.2 , we give the description of the generic construction. Then in Section 4.3, we prove the security of the proposed construction. We note that all of the projective hash families used in our construction can be constructed from a diverse group system [15]. Therefore, our proposed construction is fairly generic.

\subsection{Computationally Universal 2 Projective Hash Families}

In our generic construction of KH-PKE, a "computationally secure" variant of the notion of universal 2 projective hash families is utilized. Here we describe a formalization of the notion, which we call (first-uniform

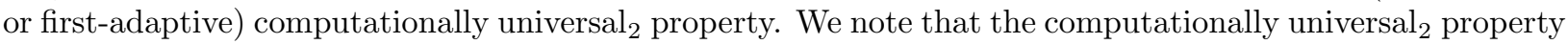
for projective hash families introduced by Hofheinz and Kiltz [28] implies the first-uniform computationally universal $_{2}$ property in our sense, therefore our definition of the notion here covers wider situations than that in the previous work.

First, we define the first-uniform version of the computationally universal ${ }_{2}$ property as follows:

Definition 4.1 (First-Uniform Computationally Universal 2 Property). Let $\mathbf{H}=\left(H, K, X, X^{\prime}, L, \Pi, S, \alpha\right)$ be a projective hash family. We say that $\mathbf{H}$ is first-uniform computationally universal ${ }_{2}$ relative to $X^{\prime}$, if for any PPT adversary $\mathcal{A}$ with oracle Hash defined below, the probability that $\mathcal{A}^{\text {Hash }}$ wins the following game

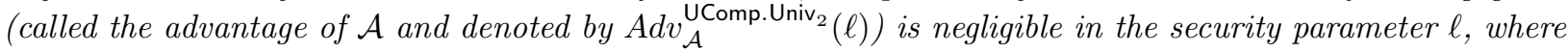
the game is as follows:

- First, the challenger generates $k \stackrel{\$}{\leftarrow} K$ and $x^{*} \stackrel{\$}{\leftarrow} X^{\prime} \backslash L$, and computes $s=\alpha(k)$ and $\pi^{*}=H_{k}\left(x^{*}\right)$. Then the challenger sends $x^{*}, s$ and $\pi^{*}$ to the adversary $\mathcal{A}$.

- During the game, the adversary can make queries Hash $(x)$ to the oracle Hash adaptively, where $x \in X$. The oracle returns $\perp$ if the input $x$ satisfies $x \in X \backslash L$, and returns $H_{k}(x)$ if $x \in L$. 
- Finally, the adversary outputs elements $x \in X$ and $\pi \in \Pi$. We define that adversary wins if and only if $x \notin L, x \neq x^{*}$ and $H_{k}(x)=\pi$.

We may omit the term "relative to $X^{\prime}$ " above when $X^{\prime}=X$. We also say that a hash proof system is firstuniform computationally universal ${ }_{2}$, if the underlying projective hash family is first-uniform computationally universal . $_{2}$

The word "first-uniform" in the definition above means that, for the two inputs $x^{*}$ and $x$ for the projective hash $H_{k}$ in the game, the first one $x^{*}$ is generated uniformly at random and the adversary cannot choose the first input. Secondly, we define the first-adaptive version of the computationally universal ${ }_{2}$ property as follows, where the adversary can choose the first input for the projective hash:

Definition 4.2 (First-Adaptive Computationally Universal 2 Property). Let $\mathbf{H}=\left(H, K, X, X^{\prime}, L, \Pi, S, \alpha\right)$ be a projective hash family. We say that $\mathbf{H}$ is first-adaptive computationally universal ${ }_{2}$, if for any PPT adversary $\mathcal{A}$ with oracle Hash defined below, the probability that $\mathcal{A}^{\text {Hash }}$ wins the following game (called the advantage of $\mathcal{A}$ and denoted by $\left.A d v_{\mathcal{A}}^{\text {AComp. Univ }_{2}}(\ell)\right)$ is negligible in the security parameter $\ell$, where the game is as follows:

- First, the challenger generates $k \stackrel{\$}{\leftarrow} K$ and computes $s=\alpha(k)$. Then the challenger sends $s$ to the adversary $\mathcal{A}$.

- During the game, the adversary can make queries Hash $(x)$ to the oracle Hash adaptively, where $x \in X$. The oracle returns $\perp$ if the input $x$ satisfies $x \in X \backslash L$, and returns $H_{k}(x)$ if $x \in L$.

- At any time in the game decided by the adversary, the adversary has to submit an element $x^{*} \in X$ to the challenger. Then the challenger returns $\pi^{*}=H_{k}\left(x^{*}\right)$ to the adversary, regardless of whether $x^{*} \in L$ or not.

- Finally, the adversary outputs elements $x \in X$ and $\pi \in \Pi$. We define that adversary wins if and only if $x, x^{*} \notin L, x \neq x^{*}$ and $H_{k}(x)=\pi$.

We also say that a hash proof system is first-adaptive computationally universal ${ }_{2}$, if the underlying projective hash family is first-adaptive computationally universal ${ }_{2}$.

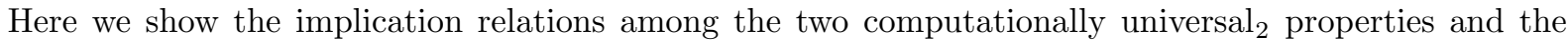
original universal ${ }_{2}$ property. Namely, we have the followings.

Lemma 4.1. If a projective hash family $\mathbf{H}$ is universal ${ }_{2}$, then $\mathbf{H}$ is first-adaptive computationally universal ${ }_{2}$.

Proof. Suppose that $\mathbf{H}$ is $\epsilon$-universal 2 for negligible $\epsilon$. Let $\mathcal{A}=\left(\mathcal{A}_{1}, \mathcal{A}_{2}\right)$ be a PPT adversary for the firstadaptive computationally universal ${ }_{2}$ game for $\mathbf{H}$, where $\mathcal{A}_{1}$ denotes the first part of $\mathcal{A}$ that takes $1^{\ell}$ and $s=\alpha(k)$ as input and outputs the submitted element $x^{*} \in X$ as well as the internal state st, and $\mathcal{A}_{2}$ denotes the second part of $\mathcal{A}$ that takes st and $\pi^{*}=H_{k}\left(x^{*}\right)$ as input and outputs the elements $x \in X$ and $\pi \in \Pi$. Namely, we have

$$
\begin{aligned}
& A d v_{\mathcal{A}}^{\text {AComp.Univ }_{2}}(\ell) \\
& =\underset{k \leftarrow K}{\operatorname{Pr}}\left[\left(x^{*}, \text { st }\right) \leftarrow \mathcal{A}_{1}^{\text {Hash }}\left(1^{\ell}, \alpha(k)\right) ;(x, \pi) \leftarrow \mathcal{A}_{2}^{\text {Hash }}\left(\text { st }, H_{k}\left(x^{*}\right)\right): x, x^{*} \notin L \wedge x \neq x^{*} \wedge H_{k}(x)=\pi\right] .
\end{aligned}
$$

This expression can be rewritten as

$$
\begin{aligned}
& A d v_{\mathcal{A}}^{\text {AComp.Univ }_{2}}(\ell) \\
& \begin{aligned}
=\sum_{s \in S} \sum_{\substack{x, x^{*} \in X \backslash L \\
x \neq x^{*}}} \sum_{\pi, \pi^{*} \in \Pi} \operatorname{Pr}_{k \leftarrow K}\left[\left(x^{* \dagger}, \text { st }\right) \leftarrow \mathcal{A}_{1}^{\text {Hash }}\left(1^{\ell}, s\right) ;\left(x^{\dagger}, \pi^{\dagger}\right) \leftarrow \mathcal{A}_{2}^{\text {Hash }}\left(\text { st }, \pi^{*}\right)\right. \\
\left.: x^{* \dagger}=x^{*} \wedge x^{\dagger}=x \wedge \pi^{\dagger}=\pi \wedge H_{k}\left(x^{*}\right)=\pi^{*} \wedge H_{k}(x)=\pi \wedge \alpha(k)=s\right]
\end{aligned}
\end{aligned}
$$


Now note that each of the algorithms $\mathcal{A}_{1}$ and $\mathcal{A}_{2}$ does not use any information on the key $k$ except the information on $s=\alpha(k)$, while the oracle Hash can be simulated (not efficiently, in general) without using $k$ (by exhaustively searching elements of $L$ and witnesses for elements of $L$ ). This implies that, the expression of the advantage of $\mathcal{A}$ is equal to

$$
\begin{aligned}
& A d v_{\mathcal{A}}^{\text {AComp.Univ }_{2}(\ell)} \\
& =\sum_{s \in S} \sum_{\substack{x, x^{*} \in X \backslash L \\
x \neq x^{*}}} \sum_{\pi, \pi^{*} \in \Pi}\left(\begin{array}{l}
\operatorname{Pr}\left[\left(x^{* \dagger}, \mathrm{st}\right) \leftarrow \mathcal{A}_{1}^{\text {Hash }}\left(1^{\ell}, s\right) ;\left(x^{\dagger}, \pi^{\dagger}\right) \leftarrow \mathcal{A}_{2}^{\text {Hash }}\left(\text { st }, \pi^{*}\right): x^{* \dagger}=x^{*} \wedge x^{\dagger}=x \wedge \pi^{\dagger}=\pi\right] . \\
\left.\cdot \operatorname{Pr}_{k \leftarrow K}\left[H_{k}\left(x^{*}\right)=\pi^{*} \wedge H_{k}(x)=\pi \wedge \alpha(k)=s\right]\right)
\end{array} .\right.
\end{aligned}
$$

Since $\mathbf{H}$ is $\epsilon$-universal ${ }_{2}$, it follows that

$$
\begin{aligned}
& A d v_{\mathcal{A}}^{\text {AComp.Univ }_{2}(\ell)} \\
& \leq \sum_{s \in S} \sum_{\substack{x, x^{*} \in X \backslash L \\
x \neq x^{*}}} \sum_{\pi, \pi^{*} \in \Pi}\left(\begin{array}{c}
\operatorname{Pr}\left[\left(x^{* \dagger}, \mathrm{st}\right) \leftarrow \mathcal{A}_{1}^{\text {Hash }}\left(1^{\ell}, s\right) ;\left(x^{\dagger}, \pi^{\dagger}\right) \leftarrow \mathcal{A}_{2}^{\text {Hash }}\left(\text { st }, \pi^{*}\right): x^{* \dagger}=x^{*} \wedge x^{\dagger}=x \wedge \pi^{\dagger}=\pi\right] . \\
\left.\cdot \epsilon \cdot \operatorname{Pr}_{k \leftarrow K}\left[H_{k}\left(x^{*}\right)=\pi^{*} \wedge \alpha(k)=s\right]\right)
\end{array} .\right.
\end{aligned}
$$

The right-hand side is equal to

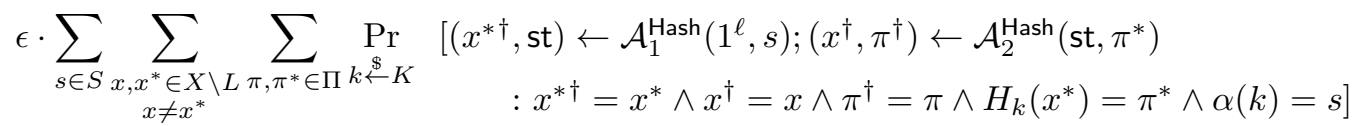

$$
\begin{aligned}
& =\epsilon \cdot \operatorname{Pr}_{k \stackrel{\$}{\$} K}\left[\left(x^{*}, \mathrm{st}\right) \leftarrow \mathcal{A}_{1}^{\text {Hash }}\left(1^{\ell}, \alpha(k)\right) ;(x, \pi) \leftarrow \mathcal{A}_{2}^{\text {Hash }}\left(\text { st }, H_{k}\left(x^{*}\right)\right): x, x^{*} \notin L \wedge x \neq x^{*}\right] \\
& \leq \epsilon
\end{aligned}
$$

Hence we have $A d v_{\mathcal{A}}^{\text {AComp.Univ }_{2}}(\ell) \leq \epsilon$ which is negligible, as desired.

Lemma 4.2. Suppose that $X^{\prime} \backslash L$ is approximately samplable relative to $X$. If a projective hash family $\mathbf{H} i s$ first-adaptive computationally universal $l_{2}$, then $\mathbf{H}$ is first-uniform computationally universal ${ }_{2}$ relative to $X^{\prime}$.

Proof. Let $\mathcal{A}$ be any PPT adversary for the first-uniform computationally universal ${ }_{2}$ game for $\mathbf{H}$ relative to $X^{\prime}$. We construct an adversary $\mathcal{A}^{\dagger}$ for the first-adaptive computationally universal ${ }_{2}$ game for $\mathbf{H}$ as follows. Given input $1^{\ell}$ and $s$ for $\mathcal{A}^{\dagger}$, the algorithm $\mathcal{A}^{\dagger}$ first samples an element $x^{*} \in X$ which is negligibly close to the uniform distribution on $X^{\prime} \backslash L$ (this can be efficiently done since $X^{\prime} \backslash L$ is approximately samplable relative to $X)$, submits $x^{*}$ to the challenger in the first-adaptive computationally universal ${ }_{2}$ game, and receives $\pi^{*}=H_{k}\left(x^{*}\right)$ by the challenger. Then $\mathcal{A}^{\dagger}$ executes $\mathcal{A}$ with input $\left(1^{\ell}, x^{*}, s, \pi^{*}\right)$, where $\mathcal{A}^{\dagger}$ simulates the oracle $\mathrm{Hash}_{U}$ in the first-uniform computationally universal 2 game in the following manner: For each query $x^{\prime}$ to Hash $_{U}$ made dy $\mathcal{A}, \mathcal{A}^{\dagger}$ makes a query $x^{\prime}$ to Hash ${ }_{A}$, receives its reply $\pi^{\prime}$ and then returns $\pi^{\prime}$ to $\mathcal{A}$ as the reply to the query. Finally, $\mathcal{A}^{\dagger}$ receives the output $(x, \pi)$ by $\mathcal{A}$, and outputs $(x, \pi)$. We note that the algorithm $\mathcal{A}^{\dagger}$ is PPT as well as $\mathcal{A}$.

To evaluate the advantage of $\mathcal{A}^{\dagger}$, we may assume without loss of generality that $x^{*}$ is a uniformly random element of $X^{\prime} \backslash L$, since the modification causes at most negligible change of the advantage of $\mathcal{A}^{\dagger}$. In the present case, the simulation by $\mathcal{A}^{\dagger}$ of the first-uniform computationally universal 2 game for $\mathcal{A}$ is perfect, and $\mathcal{A}^{\dagger}$ wins the game if and only if $\mathcal{A}$ wins. This implies that $A d v_{\mathcal{A}^{\dagger}}^{\mathrm{AComp}^{\dagger} \operatorname{Univ}_{2}}(\ell)=A d v_{\mathcal{A}}^{\text {UComp.Univ }_{2}}(\ell)$, therefore $\mathcal{A}^{\dagger}$ has non-negligible advantage whenever $\mathcal{A}$ has. Hence, the claim holds.

\subsection{The Generic Construction}

First, we summarize the primitives used in the generic construction. Let $\mathbf{M}=\left\{I_{\ell}\right\}_{\ell \geq 0}$ be a subset membership problem which specifies an instance description $\Lambda=\Lambda\left[X, X^{\prime}, L, W, R\right] \in\left[I_{\ell}\right]$. In our construction, we use the following three projective hash families $\mathbf{H}, \widehat{\mathbf{H}}$ and $\widetilde{\mathbf{H}}$, and the corresponding hash proof systems $\mathbf{P}$, $\widehat{\mathbf{P}}$ and $\widetilde{\mathbf{P}}$ associated to $\mathbf{M}$. 
- $\mathbf{H}=\left(H, K, X, X^{\prime}, L, \Pi, S, \alpha\right)$ is a homomorphic projective hash family which is smooth relative to $\left(X^{\prime}, \Pi^{\prime}\right)$, and $\mathbf{P}=$ (HPS.param, HPS.priv, HPS.pub) is the corresponding hash proof system, associated to the subset membership problem $\mathbf{M}$. In particular, $\Pi$ is an abelian group (written in additive form) and $\Pi^{\prime}$ is a subgroup of $\Pi$. Moreover, $\Pi^{\prime}$ is approximately samplable relative to $\Pi$.

- $\widehat{\mathbf{H}}=\left(\widehat{H}, \widehat{K}, X, X^{\prime}, L, \widehat{\Pi}, \widehat{S}, \widehat{\alpha}\right)$ is a homomorphic universal 1 projective hash family, and $\widehat{\mathbf{P}}=(\widehat{\mathrm{HPS}}$.param, $\widehat{\mathrm{HPS}}$. priv, $\widehat{\mathrm{HPS}}$. pub) is the corresponding hash proof system associated to $\mathbf{M}$.

- $\widetilde{\mathbf{H}}=\left(\widetilde{H}, \widetilde{K}, X \times \Pi \times \widehat{\Pi}, X^{\prime} \times \Pi \times \widehat{\Pi}, L \times \Pi \times \widehat{\Pi}, \widetilde{\Pi}, \widetilde{S}, \widetilde{\alpha}\right)$ is a computationally or information-theoretically universal ${ }_{2}$ projective hash family (see below for the detail), and $\widetilde{\mathbf{P}}=(\widetilde{\mathrm{HPS}}$. param, $\widetilde{\mathrm{HPS}}$.priv, $\widetilde{\mathrm{HPS}}$.pub) is the corresponding hash proof system.

We also introduce some additional assumptions on the objects above. For the purpose, we introduce an auxiliary terminology:

Definition 4.3. Let $\Lambda=\Lambda\left[X, X^{\prime}, L, W, R\right]$ be an instance description for $\mathbf{M}$. We say that a positive integer is a critical integer, if it is not coprime to $|X|$ and is not a multiple of $o(\Lambda)$, where $o(\Lambda)$ denotes the least common multiplier of the orders of elements of $X^{\prime}$ in the quotient group $X / L$.

Now we describe the additional assumptions mentioned above. Here we introduce three kinds of assumptions, which have the following trade-off relations: The requirement for the HPS $\widetilde{\mathbf{P}}$ is weakened in the direction Assumption I $\rightarrow$ Assumption $\mathrm{A} \rightarrow$ Assumption $\mathrm{U}$, while the other conditions is relaxed in the other direction Assumption $\mathrm{U} \rightarrow$ Assumption $\mathrm{A} \rightarrow$ Assumption I. The reason of considering the three incomparable assumptions is to cover several instantiations of the proposed generic construction under various settings discussed in later sections. Now the three assumptions are as follows:

Assumption I: $\widetilde{\mathbf{P}}$ is (information-theoretically) universal 2 .

Assumption A: $\widetilde{\mathbf{P}}$ is first-adaptive computationally universal ${ }_{2}$, and $X^{\prime} \backslash L$ is approximately samplable relative to $X$.

Assumption U: $\widetilde{\mathbf{P}}$ is first-uniform computationally universal ${ }_{2}$ relative to $X^{\prime} \times \Pi \times \widehat{\Pi}, \widehat{\mathbf{P}}$ is smooth relative to $\left(X^{\prime}, \widehat{\Pi}\right), X^{\prime} \backslash L$ is approximately samplable relative to $X$, and $\Pi^{\prime}=\Pi$. Moreover, it is computationally hard to find a critical integer from a given instance $\Lambda$ of $\mathbf{M}$ (see Definition 4.3 for the terminology); it can be efficiently checked whether a given integer is a critical integer or not; we have $x+y \in X^{\prime}$ for any $x \in X^{\prime} \backslash L$ and $y \in L$; and we have $a \cdot x \in X^{\prime}$ for any $x \in X^{\prime} \backslash L$ and any integer $a$ coprime to $|X|$.

Using these building blocks, we construct a KH-PKE scheme as in Figure 1. Roughly, the homomorphic smooth projective hash family $\mathbf{H}$ is used to hide a plaintext in a ciphertext. Moreover the universal property of $\widehat{\mathbf{H}}$ and $\widetilde{\mathbf{H}}$ are used to detect the invalidity of ciphertexts, which leads to resistance against ciphertext modification. However, the latter property looks contradictory to the homomorphic property that inherently involves such modification. In order to manage to deal with these two properties consistently, we utilize the following "transitional universal" property of the pair of $\widehat{\mathbf{H}}$ and $\widetilde{\mathbf{H}}$ :

- If an adversary does not have the secret key of $\widetilde{\mathbf{H}}$ (which is the homomorphic key), then the (computationally or information-theoretically) universal 2 property of $\widetilde{\mathbf{H}}$ can be used to reject invalid input ciphertexts for the decryption and the evaluation algorithms.

- On the other hand, if an adversary has obtained the secret key of $\widetilde{\mathbf{H}}$, then the evaluation algorithm can update the values of $\widehat{\mathbf{H}}$ and $\widetilde{\mathbf{H}}$ by using the key for $\widetilde{\mathbf{H}}$ and the homomorphic property of $\widehat{\mathbf{H}}$, while the universal 1 property of $\widehat{\mathbf{H}}$ (instead of the universal 2 property of $\widetilde{\mathbf{H}}$ which is no longer available) can be still used to reject invalid input ciphertexts for the decryption algorithm. 


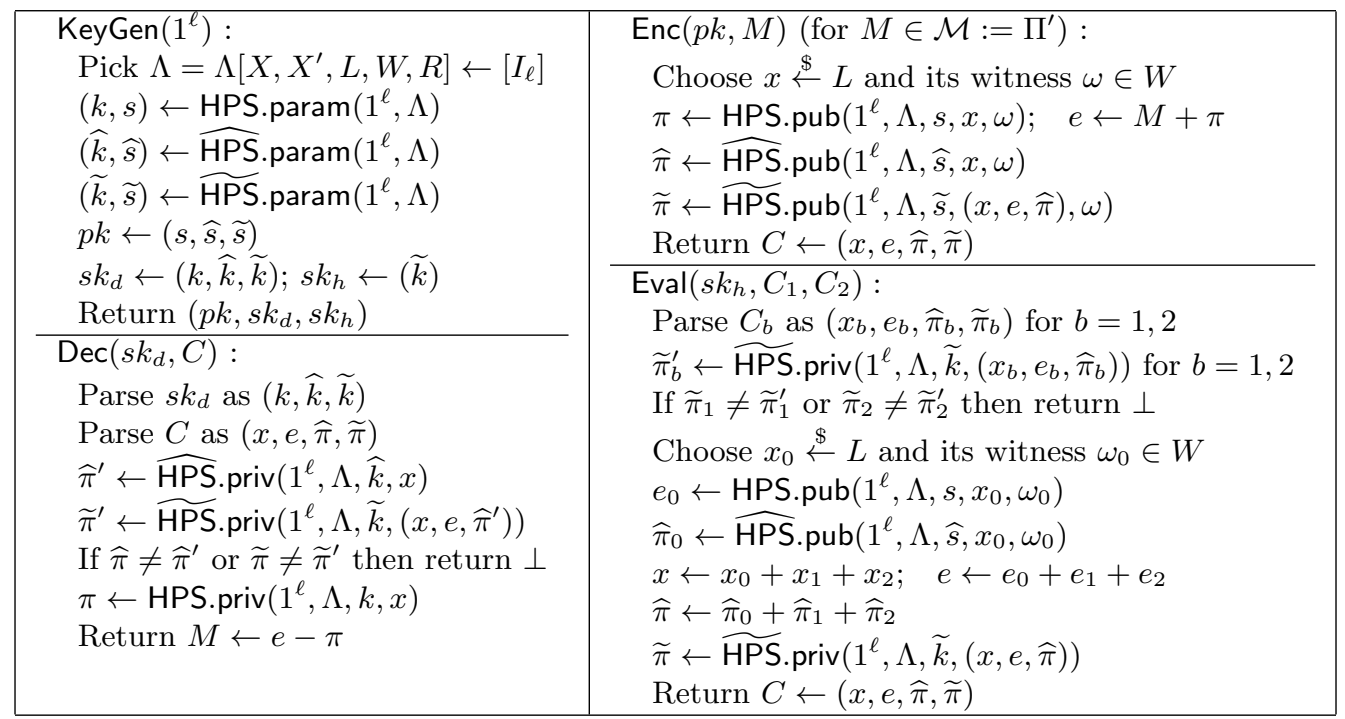

Figure 1: The proposed KH-PKE construction from HPS.

We emphasize that the evaluation algorithm first generates a fresh ciphertext $\left(x_{0}, e_{0}, \widehat{\pi}_{0}\right)$, which is identical to the first three components of a ciphertext of plaintext 0 generated by the encryption algorithm, and second performs the homomorphic operation of $C_{1}, C_{2}$ and a random ciphertext of 0 . This plays a key role in the security proof.

One might think that in the construction, $\widetilde{\mathbf{H}}$ is redundant, and thus is not necessary. However, this is not true. Namely, if $\widetilde{\mathbf{H}}$ is removed, then the adversary can extract meaningful information from the Eval oracle by submitting invalid ciphertexts, and therefore, the resulting scheme becomes insecure. In other words, with the help of $\widetilde{\mathbf{H}}$, the Eval oracle can distinguish invalid ciphertexts from valid ones, and consequently, the above attack is prevented.

Now we state the main theorem of the paper.

Theorem 4.1. Our construction above is KH-CCA-secure, if the subset membership problem $\mathbf{M}$ is hard relative to $X^{\prime} \subset X$, the hash proof systems $\mathbf{P}, \widehat{\mathbf{P}}$ and $\widetilde{\mathbf{P}}$ are as above, and one of Assumption I, Assumption $A$ and Assumption $U$ above is satisfied.

Since all of the projective hash families used in our construction can be constructed from a diverse group system, from the result of [27] (where CPA-secure homomorphic PKE (with cyclic-group ciphertext space) implies diverse group systems), the following corollary is given.

Corollary 4.1. KH-CCA secure KH-PKE is implied by CPA-secure homomorphic PKE with cyclic-group ciphertext space.

\subsection{Security Proof}

From now, we give a proof of Theorem 4.1. Here we introduce some terminology used in the proof. For a ciphertext $C=(x, e, \widehat{\pi}, \widetilde{\pi})$, we say that $C$ is regular, if $x \in L$; and irregular, if $x \in X \backslash L$. Similar terminology is used for inputs for $H_{k}, \widehat{H}_{\widehat{k}}$, and $\widetilde{H}_{\widetilde{k}}$. We say that $C$ is $\widehat{H}$-consistent (respectively, $\widetilde{H}$-consistent), if $\widehat{H}_{\widehat{k}}(x, e)=\widehat{\pi}$ (respectively, $\widetilde{H}_{\widetilde{k}}(x, e, \widehat{\pi})=\widetilde{\pi}$ ); and $\widehat{H}$ - (respectively, $\widetilde{H}$-)inconsistent otherwise. We say that $C$ is $\widehat{H}$-forging (respectively, $\widetilde{H}$-forging), if it is irregular but $\widehat{H}$-consistent (respectively, $\widetilde{H}$-consistent). We say that $C$ is valid, if it is regular, $\widehat{H}$-consistent and $\widetilde{H}$-consistent; and invalid otherwise. On the other hand, let the term private information on the secret key for a projective hash family mean any information on the key $k$ except the corresponding public information $s=\alpha(k)$. 
First, we show the correctness of the Eval algorithm. Given valid inputs $C_{1}=\left(x_{1}, e_{1}, \widehat{\pi}_{1}, \widetilde{\pi}_{1}\right)$ and $C_{2}=$ $\left(x_{2}, e_{2}, \widehat{\pi}_{2}, \widetilde{\pi}_{2}\right)$ of plaintexts $M_{1}$ and $M_{2}$, respectively, the algorithm first generates a triple $\left(x_{0}, e_{0}, \widehat{\pi}_{0}\right)$, which is identical to the first three components of a ciphertext of plaintext 0 generated by the encryption algorithm. Since $\mathbf{P}$ and $\widehat{\mathbf{P}}$ are homomorphic, by putting $x=x_{0}+x_{1}+x_{2}, e=e_{0}+e_{1}+e_{2}$ and $\widehat{\pi}=\widehat{\pi}_{0}+\widehat{\pi}_{1}+\widehat{\pi}_{2}$, we have

$$
\begin{gathered}
e=\left(0+H_{k}\left(x_{0}\right)\right)+\left(M_{1}+H_{k}\left(x_{1}\right)\right)+\left(M_{2}+H_{k}\left(x_{2}\right)\right)=\left(M_{1}+M_{2}\right)+H_{k}(x), \\
\widehat{\pi}=\widehat{H}_{\widehat{k}}\left(x_{0}\right)+\widehat{H}_{\widehat{k}}\left(x_{1}\right)+\widehat{H}_{\widehat{k}}\left(x_{2}\right)=\widehat{H}_{\widehat{k}}(x) .
\end{gathered}
$$

Therefore, $(x, e, \widehat{\pi})$ is identical to the first three components of a ciphertext of $M_{1}+M_{2}$. This implies that the output $C=(x, e, \widehat{\pi}, \widetilde{\pi})$ of the evaluation algorithm is a valid ciphertext of $M_{1}+M_{2}$, as desired.

Intuitively, the evaluation algorithm performs the homomorphic operation of $C_{1}, C_{2}$ and a random ciphertext of 0 . The reason of introducing the last random factor is to realize the following property, which plays a key role in the security proof:

Lemma 4.3 (Source Ciphertext Hiding Property). Let $\left(p k, s k_{d}, s k_{h}\right) \leftarrow \operatorname{KeyGen}\left(1^{\ell}\right), \operatorname{let} C_{2}=\left(x_{2}, e_{2}, \widehat{\pi}_{2}, \widetilde{\pi}_{2}\right)$ be any $\widetilde{H}$-consistent ciphertext, and assume that ciphertexts $C_{1}=\left(x_{1}, e_{1}, \widehat{\pi}_{1}, \widetilde{\pi}_{1}\right)$ and $C_{1}^{\prime}=\left(x_{1}^{\prime}, e_{1}^{\prime}, \widehat{\pi}_{1}^{\prime}, \widetilde{\pi}_{1}^{\prime}\right)$ are $\widetilde{H}$-consistent and satisfy the following conditions:

$$
x^{\prime}:=x_{1}^{\prime}-x_{1} \in L, e^{\prime}:=e_{1}^{\prime}-e_{1}=H_{k}\left(x^{\prime}\right) \text { and } \widehat{\pi}^{\prime}:=\widehat{\pi}_{1}^{\prime}-\widehat{\pi}_{1}=\widehat{H}_{\widehat{k}}\left(x^{\prime}, e^{\prime}\right) .
$$

As a remark, $e^{\prime}:=e_{1}^{\prime}-e_{1}=H_{k}\left(x^{\prime}\right)$ means $C_{1}$ and $C_{1}^{\prime}$ are ciphertexts of the same plaintext. Then the outputs of $\operatorname{Eval}\left(s k_{h}, C_{1}, C_{2}\right)$ and of $\operatorname{Eval}\left(s k_{h}, C_{1}^{\prime}, C_{2}\right)$ also satisfy the condition in (1), and the distributions of these two outputs of Eval are identical.

Proof. The first three components of the output of Eval $\left(s k_{h}, C_{1}, C_{2}\right)$ are of the form $x_{12}:=x_{0}+x_{1}+x_{2}$, $e_{12}:=e_{0}+e_{1}+e_{2}$ and $\widehat{\pi}_{12}:=\widehat{H}_{\widehat{k}}\left(x_{0}, e_{0}\right)+\widehat{\pi}_{1}+\widehat{\pi}_{2}$ with $x_{0} \stackrel{\$}{\leftarrow} L$ and $e_{0}=H_{k}\left(x_{0}\right)$, and the first three components of the output of $\operatorname{Eval}\left(s k_{h}, C_{1}^{\prime}, C_{2}\right)$ are of the form $x_{12}^{\prime}:=x_{0}^{\prime}+x_{1}^{\prime}+x_{2}, e_{12}^{\prime}:=e_{0}^{\prime}+e_{1}^{\prime}+e_{2}$ and $\widehat{\pi}_{12}^{\prime}:=\widehat{H}_{\widehat{k}}\left(x_{0}^{\prime}, e_{0}^{\prime}\right)+\widehat{\pi}_{1}^{\prime}+\widehat{\pi}_{2}$ with $x_{0}^{\prime} \stackrel{\$}{\leftarrow} L$ and $e_{0}^{\prime}=H_{k}\left(x_{0}^{\prime}\right)$. Now we have

$$
x_{12}^{\prime}=x_{0}^{\prime}+x_{1}^{\prime}+x_{2}=x_{0}^{\prime}+x^{\prime}+x_{1}+x_{2}=\left(x_{0}^{\prime}-x_{0}+x^{\prime}\right)+x_{12} .
$$

where $x^{\prime}:=x_{1}^{\prime}-x_{1}$. Similarly, since $\mathbf{P}$ and $\widehat{\mathbf{P}}$ are homomorphic, we have

$$
\begin{aligned}
e_{12}^{\prime} & =H_{k}\left(x_{0}^{\prime}+x^{\prime}\right)+e_{1}+e_{2}=H_{k}\left(x_{0}^{\prime}-x_{0}+x^{\prime}\right)+e_{12}=H_{k}\left(x_{12}^{\prime}-x_{12}\right)+e_{12} \\
\widehat{\pi}_{12}^{\prime} & =\widehat{H}_{\widehat{k}}\left(x_{0}^{\prime}+x^{\prime}, e_{0}^{\prime}+e^{\prime}\right)+\widehat{\pi}_{1}+\widehat{\pi}_{2} \\
& =\widehat{H}_{\widehat{k}}\left(x_{0}^{\prime}-x_{0}+x^{\prime}, e_{0}^{\prime}-e_{0}+e^{\prime}\right)+\widehat{\pi}_{12}=\widehat{H}_{\widehat{k}}\left(x_{12}^{\prime}-x_{12}, e_{12}^{\prime}-e_{12}\right)+\widehat{\pi}_{12} .
\end{aligned}
$$

Since $x^{\prime} \in L$, we have $x_{0}^{\prime}-x_{0}+x^{\prime} \in L$, therefore the condition in (1) is satisfied. Moreover, since $x_{0}, x_{0}^{\prime} \stackrel{\$}{\leftarrow}$, $e_{0}=H_{k}\left(x_{0}\right)$ and $e_{0}^{\prime}+e^{\prime}=H_{k}\left(x_{0}^{\prime}+x^{\prime}\right)$, the distributions of $\left(x_{0}^{\prime}+x^{\prime}, H_{k}\left(x_{0}^{\prime}+x^{\prime}\right), \widehat{H}_{\widehat{k}}\left(x_{0}^{\prime}+x^{\prime}, e_{0}^{\prime}+e^{\prime}\right)\right)$ and $\left(x_{0}, e_{0}, \widehat{H}_{\widehat{k}}\left(x_{0}, e_{0}\right)\right)$ are identical, so do the distributions of $\left(x_{12}, e_{12}, \widehat{\pi}_{12}\right)$ and $\left(x_{12}^{\prime}, e_{12}^{\prime}, \widehat{\pi}_{12}^{\prime}\right)$. Hence, the claim holds.

Outline of the Security Proof: Here we give an intuitive explanation of how the source ciphertext hiding property is used to prove the security. A very brief outline of the security proof is the following: First we replace the valid challenge ciphertext $C^{*}=\left(x^{*}, e^{*}, \widehat{\pi}^{*}, \widetilde{\pi}^{*}\right), x^{*} \in L$, with an invalid one with $x^{*} \in X^{\prime} \backslash L$ (owing to the hardness of the subset membership problem). Secondly, we replace the second component $e^{*}=M_{\beta}^{*}+H_{k}\left(x^{*}\right)$ with $\pi^{\dagger}+H_{k}\left(x^{*}\right)$ where $\pi^{\dagger} \in \Pi$ is statistically close to the uniformly random element of $\Pi^{\prime}$ (owing to the smoothness of $\mathbf{P}$ relative to $\left(X^{\prime}, \Pi^{\prime}\right)$ ). Then the resulting challenge ciphertext is not dependent on $M_{\beta}^{*}$ any longer, therefore any adversary has negligible advantage, as desired. However, in the proof strategy, for the step where $x^{*} \in X^{\prime} \backslash L$ and $e^{*}=M_{\beta}^{*}+H_{k}\left(x^{*}\right)$, the adversary can obtain many $\widetilde{H}$-forging ciphertexts, hence many values of $\widetilde{H}$ for irregular inputs, by applying evaluation queries to the 
Table 2: Summary of differences of games in the preliminary part; see the main text for details

\begin{tabular}{|c|c|c|c|c|}
\hline Game & pre-0 $=\mathrm{KH}-\mathrm{CCA}$ & pre-1 & pre-2 & pre- $3=0$ \\
\hline Reply to Eval & ordinary & \multicolumn{3}{|c|}{ refreshing } \\
\hline $\begin{array}{c}\text { Critical query } \\
\text { (for Assumption U) }\end{array}$ & \multicolumn{2}{|c|}{ ordinary } & \multicolumn{2}{|c|}{ rejected } \\
\hline Hash evaluation & \multicolumn{3}{|c|}{ public } & private \\
\hline $\begin{array}{l}\text { Why negligible } \\
\text { difference }\end{array}$ & $\leftarrow$ source ciphertes & $\begin{array}{r}\text { hiding } \\
\text { harc } \\
\leftarrow \text { critic } \\
\text { (see }\end{array}$ & $\begin{array}{l}\text { find } \\
\text { teger } \rightarrow \\
\text { e } 5 \text { ) }\end{array}$ & \\
\hline
\end{tabular}

irregular challenge ciphertext. In such a case, the universal ${ }_{2}$ property of $\widetilde{\mathbf{P}}$ is no longer enough to prevent the adversary to make a decryption or an evaluation query with $\widetilde{H}$-forging input ciphertext(s) and to get its reply that is dependent on private information on the secret key $k$. Then we cannot safely replace $e^{*}=M_{\beta}^{*}+H_{k}\left(x^{*}\right)$ with $e^{*}=\pi^{\dagger}+H_{k}\left(x^{*}\right)$ even by utilizing the smoothness of $\mathbf{P}$. In short, the problem here is that the replies to the evaluation queries may in general depend on the challenge ciphertext (which is switched from regular to irregular in the proof); this is the reason why, despite the similarity of our proposed construction to the Cramer-Shoup PKE scheme, a straightforward extension of the original proof strategy for CCA security of the Cramer-Shoup scheme is not sufficient for the security proof of our scheme.

Our new idea to resolve the aforementioned problem specific to our case is the following: We modify the security game in such a way that, when an evaluation query involves the challenge ciphertext as input, the challenger first generates a fresh ciphertext (which we call source ciphertext in the proof) of the same challenge plaintext, and then proceeds the remaining calculation of the query by using the source ciphertext instead of the challenge ciphertext. In the starting case of the proof where $x^{*} \in L$, the source ciphertext hiding property implies that the output distribution of the evaluation query calculated from the source ciphertext is identical to that calculated from the challenge ciphertext, therefore the modification of the game does not affect the advantage of the adversary. On the other hand, after the modification of the game where $x^{*} \in X^{\prime} \backslash L$, the challenge ciphertext becomes invalid but each source ciphertext is kept valid. This prevents the adversary to obtain additional values of $\widetilde{\mathbf{P}}$ with irregular inputs by using the evaluation queries as above; this implies that the universal 2 property of $\widetilde{\mathbf{P}}$ is still sufficient to achieve the security. (In fact, we should also introduce the replacement of the challenge ciphertext with a source ciphertext, not only for the cases of evaluation queries involving the challenge ciphertext, but also for the cases where an evaluation query involves a ciphertext related to the challenge ciphertext; see the proof below for the detail.)

Based on the discussion above, we start the proof of our main theorem.

Proof of Theorem 4.1. Let $\mathcal{A}$ be a PPT adversary against the KH-CCA game. We show that the advantage $A d v_{\mathrm{KH}-\mathrm{KKE}, \mathcal{A}}^{\mathrm{KH}}(\ell)$ of $\mathcal{A}$ is negligible. First note that, since $\mathcal{A}$ is PPT, the total number of queries made by $\mathcal{A}$ is bounded by a polynomial $Q=Q(\ell)$. We use game-hopping from the original KH-CCA game to the ideal situation that the challenge bit $\beta$ is not used during the game (hence the advantage is zero). The game-hopping consists of the preliminary part and the main part.

Preliminary part of the game-hopping: We start with the KH-CCA game (Game pre-0). First we replace the challenge ciphertext involved in each evaluation query with a fresh ciphertext, as mentioned before the proof (Game pre-1). Then, in order to deal with irregular ciphertexts, we use the private evaluation algorithms for $\mathbf{P}, \widehat{\mathbf{P}}$, and $\widetilde{\mathbf{P}}$ in Enc instead of the public evaluation algorithms (Game pre-3). Moreover, for the case of Assumption U, we also introduce an additional technical step (Game pre-2) between Game pre-1 and Game pre-3 to avoid a problem caused later by certain evaluation queries related to critical integers, by making such queries automatically rejected. Detailed descriptions of the games are as follows (see Table 2 for a summary). In the proof, let $T_{i}$ denote the event that Game $i$ outputs 1 . 
Game pre-0: This game simulates the KH-CCA game. We give notational remarks: Let $M_{0}^{*}, M_{1}^{*}$ denote the challenge plaintexts, let $\beta$ denote the challenge bit, and let $C^{*}=\left(x^{*}, e^{*}, \widehat{\pi}^{*}, \widetilde{\pi}^{*}\right)$ denote the challenge ciphertext generated by $C^{*} \leftarrow \operatorname{Enc}\left(p k, M_{\beta}^{*}\right)$. We say that the challenger rejects a query, if the reply to the query is $\perp$. Then, the game outputs 1 if the guessing bit $\beta^{\prime}$ output by the adversary in the simulated KH-CCA game is equal to $\beta$, and outputs 0 otherwise.

We note that the complexity of the game is polynomial, and $\left|\operatorname{Pr}\left[T_{\text {pre- } 0}\right]-1 / 2\right|=A d v_{\text {KH-PKE, } \mathcal{A}}^{\mathrm{KH}}(\ell)$.

Game pre-1: In comparison to Game pre-0, in guess stage, we introduce another auxiliary dictionary $\mathcal{D}^{\prime}$ and modify the rule for the challenger to reply to evaluation queries $\left(C^{\prime}, C^{\prime \prime}\right)$ satisfying that at least one of $C^{\prime}$ and $C^{\prime \prime}$ is listed in the original dictionary $\mathcal{D}$ and the query is not rejected (i.e., RevHK has not been queried, and any of the two input ciphertexts that is not in $\mathcal{D}$ is $\widetilde{H}$-consistent; note that any ciphertext in $\mathcal{D}$ passes the test by the construction of Eval). When $\mathcal{D}=\left(C_{0}, C_{1}, \ldots, C_{\kappa}\right)$ where $C_{0}=C^{*}$ and $C_{1}, \ldots, C_{\kappa}$ were added to $\mathcal{D}$ in this order, $\mathcal{D}^{\prime}$ is of the form $\left(\left(D_{1}^{\prime}, D_{1}^{\prime \prime}\right), \ldots,\left(D_{\kappa}^{\prime}, D_{\kappa}^{\prime \prime}\right)\right)$ where each of $D_{i}^{\prime}$ and $D_{i}^{\prime \prime}$ is either an $\widetilde{H}$-consistent ciphertext or an index in $\{0,1, \ldots, i-1\}$. (We note that $\mathcal{D}^{\prime}$ is empty at the beginning of the guess stage where $\mathcal{D}=\left(C^{*}\right)$.) Intuitively, the content of $\mathcal{D}^{\prime}$ means that $C_{i}$ was the reply to the evaluation query $\left(D_{i}^{\prime}, D_{i}^{\prime \prime}\right)$ where, if $D_{i}^{\prime}$ or $D_{i}^{\prime \prime}$ is an index $j$, then it is interpreted as the content $C_{j}$ of $\mathcal{D}$.

Now we describe the modified rule for the challenger to reply to the next evaluation query of the form $\left(C^{\prime}, C^{\prime \prime}\right)$ as above, where $\mathcal{D}=\left(C_{0}, C_{1}, \ldots, C_{\kappa}\right)$ and $\mathcal{D}^{\prime}=\left(\left(D_{1}^{\prime}, D_{1}^{\prime \prime}\right), \ldots,\left(D_{\kappa}^{\prime}, D_{\kappa}^{\prime \prime}\right)\right)$. We call it the $(\kappa+1)$ th refreshing process in the sequel, and we also call the query the $(\kappa+1)$-th refreshing query. In the process, the challenger first generates auxiliary ciphertexts $\bar{C}_{0}{ }^{(\kappa+1)}=\bar{C}^{*}(\kappa+1), \bar{C}_{1}^{(\kappa+1)}, \ldots, \bar{C}_{\kappa}{ }^{(\kappa+1)}$ as follows:

- First, the challenger generates $\bar{C}^{*}(\kappa+1) \leftarrow \operatorname{Enc}\left(p k, M_{\beta}^{*}\right)$ instead of using $C^{*}$ itself, which we call the source ciphertext for the refreshing process. Then for each $i=1, \ldots, \kappa$, the challenger generates $\bar{C}_{i}^{(\kappa+1)}$ by using Eval $\left(s k_{h}, \cdot, \cdot\right)$, where its second (respectively, third) input is $D_{i}^{\prime}$ (respectively, $D_{i}^{\prime \prime}$ ) if $D_{i}^{\prime}$ (respectively, $\left.D_{i}^{\prime \prime}\right)$ is a ciphertext (i.e., not an index), and it is $\bar{C}_{j}^{(\kappa+1)}$ if $D_{i}^{\prime}$ (respectively, $D_{i}^{\prime \prime}$ ) is an index $j \in\{0,1, \ldots, i-1\}$.

Secondly, the challenger sets $D_{\kappa+1}^{\prime}$ to be $C^{\prime}$ if $C^{\prime} \notin \mathcal{D}$, and to be an index $i$ if $C^{\prime} \in \mathcal{D}$ and $i$ is the smallest index satisfying $C^{\prime}=C_{i}$. The challenger also determines $D_{\kappa+1}^{\prime \prime}$ similarly by using $C^{\prime \prime}$ instead of $C^{\prime}$. Thirdly, the challenger generates $C_{\kappa+1}$ by using $\operatorname{Eval}\left(s k_{h}, \cdot, \cdot\right)$, where its second (respectively, third) input is $D_{\kappa+1}^{\prime}$ (respectively, $D_{\kappa+1}^{\prime \prime}$ ) if $D_{\kappa+1}^{\prime}$ (respectively, $D_{\kappa+1}^{\prime \prime}$ ) is a ciphertext, and it is $\bar{C}_{i}{ }^{(\kappa+1)}$ if $D_{\kappa+1}^{\prime}$ (respectively, $\left.D_{\kappa+1}^{\prime \prime}\right)$ is an index $i \in\{0,1, \ldots, \kappa\}$. Finally, the challenger adds $C_{\kappa+1}$ to $\mathcal{D}$, adds $\left(D_{\kappa+1}^{\prime}, D_{\kappa+1}^{\prime \prime}\right)$ to $\mathcal{D}^{\prime}$ and gives $C_{\kappa+1}$ to the adversary as the reply to the evaluation query.

By using the source ciphertext hiding property recursively, the distribution of $C_{\kappa+1}$ in the modified rule becomes identical to that of $C_{\kappa+1}$ in the original rule (note that any two outputs of $\operatorname{Enc}\left(p k, M_{\beta}^{*}\right)$ satisfy the condition (1)). This implies that the distribution of the adversary's view is identical in the two cases, therefore we have $\operatorname{Pr}\left[T_{\text {pre- } 1}\right]=\operatorname{Pr}\left[T_{\text {pre-0 }}\right]$. We note that the (time and memory) complexity of this game is still polynomial, since the number of evaluation queries made by $\mathcal{A}$ is bounded by the polynomial $Q$ and the complexity of each refreshing process is linear in $\kappa$.

Game pre-2: We need the game only for the case of Assumption U (in the other case, we set the game to be identical to Game pre-1). First we introduce some auxiliary definitions. For the dictionary $\mathcal{D}=\left(C_{0}=\right.$ $\left.C^{*}, C_{1}, \ldots, C_{\kappa}\right)$ and a ciphertext $C$, we define $\iota_{\mathcal{D}}(C)=h$ if $C \in \mathcal{D}$ and $h$ is the smallest index with $C=C_{h}$, and $\iota_{\mathcal{D}}(C)=\perp$ if $C \notin \mathcal{D}$. Moreover, for an index $h \in\{0,1, \ldots, \kappa\}$, we define a positive integer $\lambda_{\mathcal{D}}(h)$ in the following manner: We set $\lambda_{\mathcal{D}}(0)=1$, and for $h>0$, if $C_{h}$ was the reply to an evaluation query $\left(C^{\prime}, C^{\prime \prime}\right)$ where either $C^{\prime}$ or $C^{\prime \prime}$ was in $\mathcal{D}$, then we set $\lambda_{\mathcal{D}}(h)=\lambda^{\prime}+\lambda^{\prime \prime}$ where $\lambda^{\prime}=\lambda_{\mathcal{D}}\left(\iota_{\mathcal{D}}\left(C^{\prime}\right)\right)$ if $C^{\prime} \in \mathcal{D}$ and $\lambda^{\prime}=0$ if $C^{\prime} \notin \mathcal{D}$, and $\lambda^{\prime \prime}$ is similarly defined by using $C^{\prime \prime}$ instead of $C^{\prime}$. Intuitively, the integer $\lambda_{\mathcal{D}}(h)$ indicates how many copies of the challenge ciphertext $C^{*}$ were added in the calculation of the ciphertext $C_{h}$ in $\mathcal{D}$.

Based on the definition, we modify Game pre-1 in such a way that any evaluation query $\left(C^{\prime}, C^{\prime \prime}\right)$ satisfying the condition for a refreshing query and that $C^{\prime} \in \mathcal{D}, C^{\prime \prime} \in \mathcal{D}$ and $\lambda_{\mathcal{D}}\left(\iota_{\mathcal{D}}\left(C^{\prime}\right)\right)+\lambda_{\mathcal{D}}\left(\iota_{\mathcal{D}}\left(C^{\prime \prime}\right)\right)$ is a critical integer is always rejected (we call such a query a critical query); in the sequel, we exclude each critical query from the refreshing queries. 
Table 3: Summary of differences of games in the main part; see the main text for details

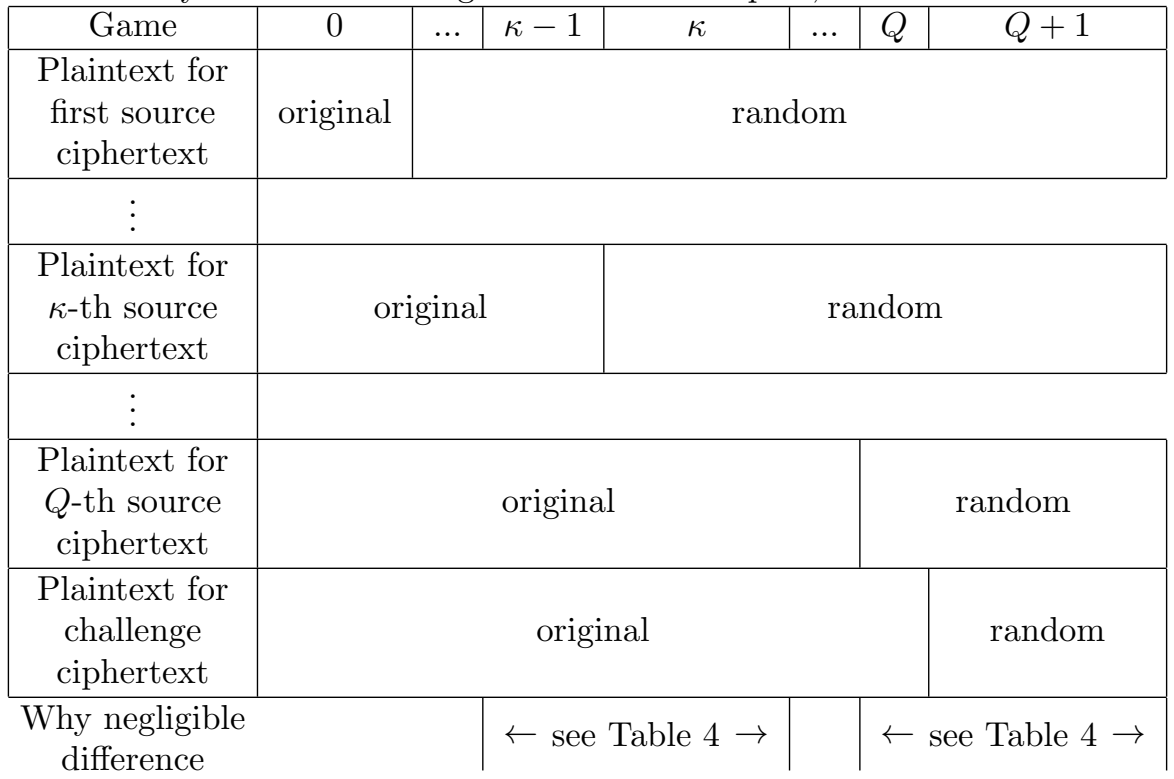

By the definition, the difference $\left|\operatorname{Pr}\left[T_{\text {pre-2 }}\right]-\operatorname{Pr}\left[T_{\text {pre-1 }}\right]\right|$ will be evaluated owing to the hardness of finding a critical integer (see Assumption U). In order to carefully analyze the behaviors of the games, we will introduce subdivision of the game-hopping, SubGames pre-2.0 to pre-2.Q that connect Game pre- 1 to Game pre-2, where any of the first $\kappa$ evaluation queries $(0 \leq \kappa \leq Q)$ is rejected if it is a critical query. Details will be described later. We note that the complexity of the game is polynomial, owing to the efficiency of deciding whether a given integer is a critical integer or not (see Assumption U).

Game pre-3: Recall that, in the algorithm Enc, values of $\mathbf{P}, \widehat{\mathbf{P}}$, and $\widetilde{\mathbf{P}}$ are computed by using the public evaluation algorithms and witnesses of elements of $L$. In the game, we modify Game pre-2 in such a way that the challenger executes Enc by using the private evaluation algorithms of $\mathbf{P}, \widehat{\mathbf{P}}$ and $\widetilde{\mathbf{P}}$ and their secret keys, where witnesses of elements of $L$ are no longer required. To avoid confusion, we write the modified algorithm by Enc' from now. Since the modification does not change the output distributions of the encryption algorithms, we have $\operatorname{Pr}\left[T_{\text {pre-3 }}\right]=\operatorname{Pr}\left[T_{\text {pre-2 }}\right]$. We note that the complexity of the game is still polynomial.

Main part of the game-hopping: In Game pre-3, the source ciphertexts in the refreshing queries have been made fresh and their first components have been made independent of the challenge ciphertext. Owing to this, now the replacement of the first component $x^{*} \in L$ of the challenge ciphertext with $x^{*} \in X^{\prime} \backslash L$ performed in the following game-hopping does not affect the behaviors of the refreshing queries, as desired. However, as a trade-off, now not only the challenge ciphertext but also the source ciphertexts involve information on $M_{\beta}^{*}$. From now, we proceed the game-hopping to remove the information on $\beta$ from the source ciphertexts one by one as well as from the challenge ciphertext. The process is performed by the following sequence of Games $0,1, \ldots, Q,(Q+1)$, where Game 0 is identical to Game pre-3 (see Table 3 for a summary):

Game $\kappa(0 \leq \kappa \leq Q)$ : In comparison to Game pre-3, the constructions of the source ciphertexts $\bar{C}^{*}\left(\kappa^{\prime}\right)$ in the first $\kappa$ refreshing processes, $0 \leq \kappa^{\prime} \leq \kappa$, are modified as follows: ${ }^{1}$ The second component of $\bar{C}^{*}\left(\kappa^{\prime}\right)$ is chosen as $e^{*\left(\kappa^{\prime}\right)} \leftarrow \pi^{\dagger}+\mathrm{HPS}$. priv $\left(1^{\bar{\ell}}, \Lambda, k, x^{*\left(\kappa^{\prime}\right)}\right)$, instead of $e^{*\left(\kappa^{\prime}\right)} \leftarrow M_{\beta}^{*}+\mathrm{HPS}$. priv $\left(1^{\ell}, \Lambda, k, x^{*\left(\kappa^{\prime}\right)}\right)$ as in the algorithm Enc', where $x^{*\left(\kappa^{\prime}\right)}$ is the first component of $\bar{C}^{*}\left(\kappa^{\prime}\right)$ and $\pi^{\dagger} \in \Pi$ is chosen independently of $\beta$

\footnotetext{
${ }^{1}$ When there are only less than $\kappa$ refreshing processes, we simply ignore the case of $\kappa^{\prime}$ beyond the number of the refreshing processes. Similar remarks are applied to the following arguments.
} 
Table 4: Summary of differences of games from Game $(\kappa-1)$ to Game $\kappa$ (when $\kappa=Q+1$, the challenge ciphertext is focused on instead of source ciphertexts); see the main text for details

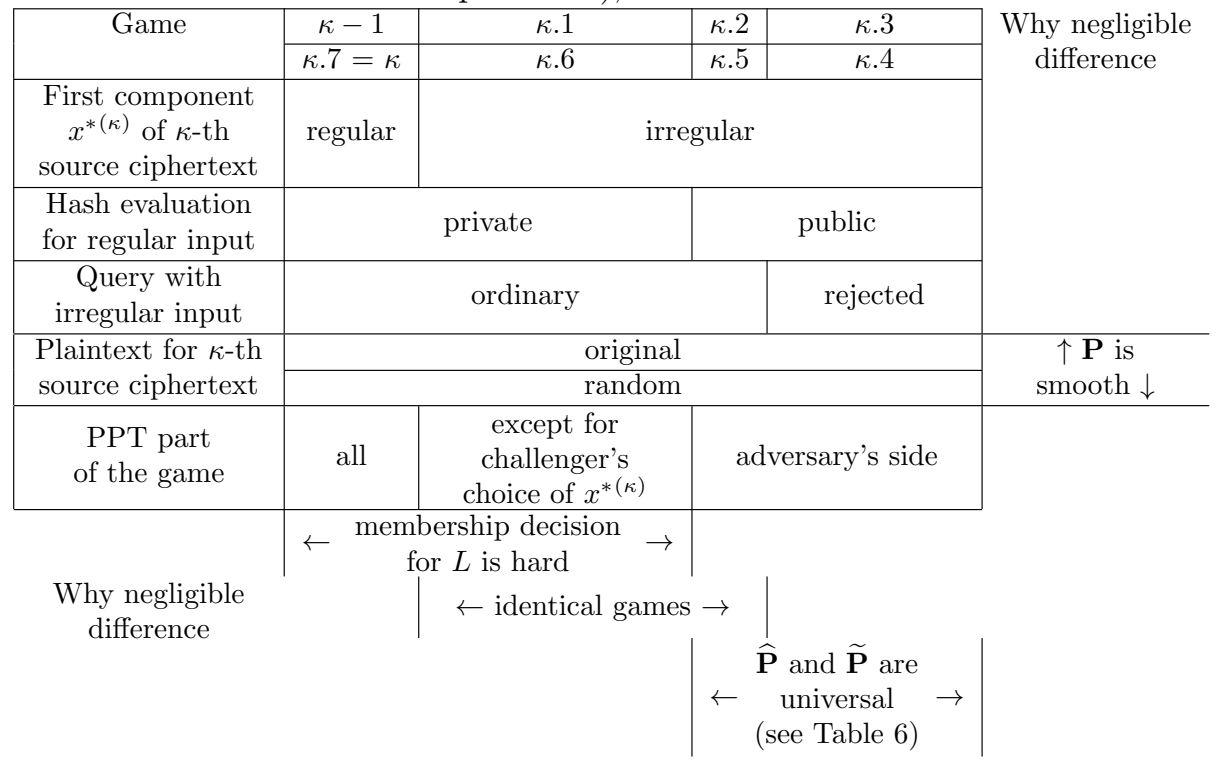

according to the probability distribution which is negligibly close to the uniform distribution on $\Pi^{\prime}$ (note that $\Pi^{\prime}$ is approximately samplable relative to $\left.\Pi\right)$. We note that the complexity of the game is polynomial.

Game $(Q+1)$ : In comparison to Game $Q$, the construction of the challenge ciphertext $C^{*}=\left(x^{*}, e^{*}, \widehat{\pi}^{*}, \tilde{\pi}^{*}\right)$ is modified as follows: The second component of $C^{*}$ is chosen as $e^{*} \leftarrow \pi^{\dagger}+$ HPS.priv $\left(1^{\ell}, \Lambda, k, x^{*}\right)$, instead of $e^{*} \leftarrow M_{\beta}^{*}+$ HPS.priv $\left(1^{\ell}, \Lambda, k, x^{*}\right)$ as in Game $Q$, where $\pi^{\dagger} \in \Pi$ is chosen independently of $\beta$ according to the probability distribution which is negligibly close to the uniform distribution on $\Pi^{\prime}$ (note that $\Pi^{\prime}$ is approximately samplable relative to $\Pi$ ).

We note that Game $(Q+1)$ is the goal of our game-hopping, where the information on the challenge bit has been removed from the replies to all the (at most) $Q$ refreshing queries and from the challenge ciphertext, therefore the advantage of the adversary becomes zero. In order to evaluate the differences of probabilities $\operatorname{Pr}\left[T_{\kappa}\right]$ between these games later, we introduce the following subdivision of the game sequence that connects each Game $(\kappa-1)$ to Game $\kappa$. An outline is as follows: We replace the first component $x^{*(\kappa)} \in L$ of the source ciphertext $\bar{C}^{*}(\kappa)$ with $x^{*(\kappa)} \in X^{\prime} \backslash L$ owing to the hardness of the subset membership problem (SubGame $\kappa .1)$, and then the second component $e^{*(\kappa)}$ of $\bar{C}^{*(\kappa)}$ is chosen as $e^{*(\kappa)} \leftarrow \pi^{\dagger}+$ HPS. priv $\left(1^{\ell}, \Lambda, k, x^{*(\kappa)}\right)$ instead of $e^{*(\kappa)} \leftarrow M_{\beta}^{*}+$ HPS.priv $\left(1^{\ell}, \Lambda, k, x^{*(\kappa)}\right)$ owing to the smoothness of $\mathbf{P}$ (SubGame $\kappa .4$ ). In order to utilize the smoothness of $\mathbf{P}$, we should guarantee that the private information on the keys for $\mathbf{P}$ are not used at any other step. For the purpose, before utilizing the smoothness of $\mathbf{P}$, we modify the game in such a way that all queries involving irregular ciphertexts are automatically rejected, owing to the universal properties of $\widehat{\mathbf{P}}$ and $\widetilde{\mathbf{P}}$ (SubGame $\kappa .2$ and SubGame $\kappa .3$ ). Moreover, after the replacement of the choice of $e^{*(\kappa)}$ as above, we restore the modifications introduced in SubGame $\kappa .1$ to SubGame $\kappa .3$ to the original situation (SubGame $\kappa .5$ to SubGame $\kappa .7)$. When $\kappa=Q+1$, similar modifications are applied to the challenge ciphertext instead of the source ciphertexts. The precise description is as follows (see Table 4 for a summary):

SubGame $\kappa .1$ : In the game, we modify the construction in Game $(\kappa-1)$ of the source ciphertext $\bar{C}^{*}(\kappa)$ in the following manner (when $\kappa=Q+1$, we apply the same modification by focusing on the challenge ciphertext $C^{*}$ instead of the source ciphertexts): For the source ciphertext $\bar{C}^{*}(\kappa)=\left(x^{*(\kappa)}, e^{*(\kappa)}, \widehat{\pi}^{*(\kappa)}, \widetilde{\pi}^{*(\kappa)}\right)$ originally generated by using Enc', the first component $x^{*(\kappa)}$ is chosen uniformly at random from $X^{\prime} \backslash L$ instead of $L$. Then the complexity of the game is polynomial except for the challenger's choice of $x^{*(\kappa)}$. Now 
we can bound the difference $\left|\operatorname{Pr}\left[T_{\kappa .1}\right]-\operatorname{Pr}\left[T_{\kappa-1}\right]\right|$ owing to the hardness of the subset membership problem; we will give a detailed argument later.

SubGame $\kappa .2$ : In the game, we modify SubGame $\kappa .1$ in the following manner: In algorithms Enc', Dec and Eval, the challenger computes the values of $\mathbf{P}, \widehat{\mathbf{P}}$, and $\widetilde{\mathbf{P}}$ for regular inputs by first exhaustively searching a witness for the first component of the input and then using the public evaluation algorithms and the witness instead of the private evaluation algorithms. To avoid confusion, we denote the modified algorithms by Enc", $\mathrm{Dec}^{\prime \prime}$ and Eval", respectively. This modification aims at clarifying that private information on the keys for HPSs are not used to compute their values for regular inputs, and this helps us to prove Claim 1 later. ${ }^{2}$ We note that still the private evaluation algorithms are used for irregular inputs as in the previous games. Now the projective property of the projective hash family implies that the computed value is not changed by the modification, therefore we have $\operatorname{Pr}\left[T_{\kappa .2}\right]=\operatorname{Pr}\left[T_{\kappa .1}\right]$.

SubGame $\kappa .3$ : In comparison to SubGame $\kappa .2$, in the game, we modify the rule to decide in which case the challenger rejects each decryption or evaluation query made by $\mathcal{A}$ in such a way that any query with irregular input that is not in the dictionary $\mathcal{D}$ is automatically rejected. From now, we refer to the new rule as the enhanced rejection rule, while we refer to the original rule as the original rejection rule.

In the game, the complexity of the adversary alone is still polynomial. The difference $\operatorname{Pr}\left[T_{\kappa .3}\right]-\operatorname{Pr}\left[T_{\kappa .2}\right]$ can be evaluated owing to the computationally or information-theoretically universal properties of $\widehat{\mathbf{P}}$ and $\widetilde{\mathbf{P}}$. In fact, in order to carefully analyze the behaviors of the games, we will introduce further subdivision of the game-hopping, SubSubGames $\kappa .3 .0$ to $\kappa .3 . Q$ that connect SubGame $\kappa .2$ to SubGame $\kappa .3$, where the rejection rule for one query is replaced at each step of the subdivided game-hopping. Details will be described later.

SubGame $\kappa .4$ : In the game, we modify the construction in SubGame $\kappa .3$ of the source ciphertext $\bar{C}^{*}(\kappa)=$ $\left(x^{*(\kappa)}, e^{*(\kappa)}, \widehat{\pi}^{*(\kappa)}, \widetilde{\pi}^{*(\kappa)}\right)$ in the $\kappa$-th refreshing process as follows (when $\kappa=Q+1$, we apply the same modification by focusing on the challenge ciphertext $C^{*}$ instead of the source ciphertexts): The second component is chosen as $e^{*(\kappa)} \leftarrow \pi^{\dagger}+\mathrm{HPS} \cdot \operatorname{priv}\left(1^{\ell}, \Lambda, k, x^{*(\kappa)}\right)$, instead of $e^{*(\kappa)} \leftarrow M_{\beta}^{*}+\mathrm{HPS} \cdot \operatorname{priv}\left(1^{\ell}, \Lambda, k, x^{*(\kappa)}\right)$ as in SubGame $\kappa .3$, where $\pi^{\dagger} \in \Pi$ is chosen independently of $\beta$ according to the probability distribution which is negligibly close to the uniform distribution on $\Pi^{\prime}$ (note that $\Pi^{\prime}$ is approximately samplable relative to $\Pi)$.

We can show that, the private information on the key $k$ for $\mathbf{P}$ is not used for irregular input in SubGame $\kappa .3$ except in the computation of $e^{*(\kappa)}=M_{\beta}^{*}+\mathrm{HPS} \cdot \operatorname{priv}\left(1^{\ell}, \Lambda, k, x^{*(\kappa)}\right)\left(\right.$ or of $e^{*}=M_{\beta}^{*}+\mathrm{HPS} \cdot \operatorname{priv}\left(1^{\ell}, \Lambda, k, x^{*}\right)$, when $\kappa=Q+1)$, and the smoothness of $\mathbf{P}$ relative to $\left(X^{\prime}, \Pi^{\prime}\right)$ implies that the difference $\left|\operatorname{Pr}\left[T_{\kappa .4}\right]-\operatorname{Pr}\left[T_{\kappa .3}\right]\right|$ is negligible. A detailed argument will be given later.

SubGame $\kappa .5$ : This game is identical to SubGame $\kappa .2$, except for the modification made from SubGame $\kappa .3$ to SubGame $\kappa .4$ for the plaintext for $\kappa$-th source ciphertext (or the challenge ciphertext, when $\kappa=Q+1$ ). That is, we restore the rejection rule from the enhanced one to the original one. In the same way as SubGame $\kappa .3$, the difference $\operatorname{Pr}\left[T_{\kappa .5}\right]-\operatorname{Pr}\left[T_{\kappa .4}\right]$ can be evaluated owing to the computationally or informationtheoretically universal properties of $\widehat{\mathbf{P}}$ and $\widetilde{\mathbf{P}}$. We will introduce further subdivision of the game-hopping, SubSubGames $\kappa .5 .0$ to $\kappa .5 . Q$ that connect SubGame $\kappa .4$ to SubGame $\kappa .5$, where the rejection rule for one query is restored at each step. Details will be described later.

SubGame $\kappa .6$ : This game is identical to SubGame $\kappa .1$, except for the modification made from SubGame $\kappa .2$ to SubGame $\kappa .4$ for the plaintext for $\kappa$-th source ciphertext (or the challenge ciphertext, when $\kappa=Q+1$ ). That is, the rejection rule is the original one, and algorithms Enc', Dec and Eval are used instead of using Enc $^{\prime \prime}$, Dec $^{\prime \prime}$ and Eval", respectively. In the same way as SubGame $\kappa .2$, the projective property of the projective hash family implies again that $\operatorname{Pr}\left[T_{\kappa .6}\right]=\operatorname{Pr}\left[T_{\kappa .5}\right]$.

\footnotetext{
${ }^{2}$ We remark that such a transition is acceptable if we make an information-theoretic jump, i.e., Assumption I. However, we still require that, for Assumptions A and U, PPT adversaries need to use the private evaluation algorithms of $\mathbf{P}$ and $\widehat{\mathbf{P}}$ for regular input by using $k$ and $\widehat{k}$ since we use computationally universal properties. However, again the projective property of the projective hash family implies that the computed value is not changed by the modification, and thus still the simulation works well. See the description of adversaries $\mathcal{A}_{3,1}$ and $\mathcal{A}_{3,2}$ later.
} 
SubGame $\kappa .7$ : This game is identical to Game $(\kappa-1)$, except for the modification made from SubGame $\kappa .1$ to SubGame $\kappa .4$ for the plaintext for $\kappa$-th source ciphertext (or the challenge ciphertext, when $\kappa=Q+1$ ). That is, the rejection rule is the original one, algorithms Enc', Dec and Eval are used, and $x^{*(\kappa)}$ is chosen uniformly at random from $L$. Namely, this game is nothing but Game $\kappa$. In the same way as SubGame $\kappa .1$, we can bound the difference $\left|\operatorname{Pr}\left[T_{\kappa .7}\right]-\operatorname{Pr}\left[T_{\kappa .6}\right]\right|$ owing to the hardness of the subset membership problem. We will give a detailed argument later.

In Game $(Q+1)$, the information on the challenge bit $\beta$ is not used during the game, which implies that $\operatorname{Pr}\left[T_{Q+1}\right]=1 / 2$. This is the goal of the game-hopping. Then we have

$$
A d v_{\mathrm{KH}-\mathrm{PKE}, \mathcal{A}}^{\mathrm{KH}}(\ell)=\left|\operatorname{Pr}\left[T_{\text {pre- } 0}\right]-1 / 2\right|=\left|\operatorname{Pr}\left[T_{\text {pre- } 0}\right]-\operatorname{Pr}\left[T_{Q+1}\right]\right| .
$$

Now, since we have mentioned that the differences for some steps of the game-hopping are zero, we have

$$
\begin{aligned}
& \operatorname{Pr}\left[T_{\text {pre-0 }}\right]-\operatorname{Pr}\left[T_{Q+1}\right] \\
& =\left(\operatorname{Pr}\left[T_{\text {pre- } 0}\right]-\operatorname{Pr}\left[T_{\text {pre- } 1}\right]\right)+\left(\operatorname{Pr}\left[T_{\text {pre- } 1}\right]-\operatorname{Pr}\left[T_{\text {pre- } 2}\right]\right)+\left(\operatorname{Pr}\left[T_{\text {pre- } 2}\right]-\operatorname{Pr}\left[T_{\text {pre- } 3}\right]\right) \\
& \quad+\sum_{\kappa=1}^{Q+1}\left(\left(\operatorname{Pr}\left[T_{\kappa-1}\right]-\operatorname{Pr}\left[T_{\kappa .1}\right]\right)+\left(\operatorname{Pr}\left[T_{\kappa .1}\right]-\operatorname{Pr}\left[T_{\kappa .2}\right]\right)+\left(\operatorname{Pr}\left[T_{\kappa .2}\right]-\operatorname{Pr}\left[T_{\kappa .3}\right]\right)+\left(\operatorname{Pr}\left[T_{\kappa .3}\right]-\operatorname{Pr}\left[T_{\kappa .4}\right]\right)\right. \\
& \left.\quad+\left(\operatorname{Pr}\left[T_{\kappa .4}\right]-\operatorname{Pr}\left[T_{\kappa .5}\right]\right)+\left(\operatorname{Pr}\left[T_{\kappa .5}\right]-\operatorname{Pr}\left[T_{\kappa .6}\right]\right)+\left(\operatorname{Pr}\left[T_{\kappa .6}\right]-\operatorname{Pr}\left[T_{\kappa}\right]\right)\right) \\
& =\left(\operatorname{Pr}\left[T_{\text {pre-1 }}\right]-\operatorname{Pr}\left[T_{\text {pre- } 2}\right]\right)+\sum_{\kappa=1}^{Q+1}\left(\left(\operatorname{Pr}\left[T_{\kappa-1}\right]-\operatorname{Pr}\left[T_{\kappa .1}\right]\right)+\left(\operatorname{Pr}\left[T_{\kappa .2}\right]-\operatorname{Pr}\left[T_{\kappa .3}\right]\right)+\left(\operatorname{Pr}\left[T_{\kappa .3}\right]-\operatorname{Pr}\left[T_{\kappa .4}\right]\right)\right. \\
& \left.\quad+\left(\operatorname{Pr}\left[T_{\kappa .4}\right]-\operatorname{Pr}\left[T_{\kappa .5}\right]\right)+\left(\operatorname{Pr}\left[T_{\kappa .6}\right]-\operatorname{Pr}\left[T_{\kappa}\right]\right)\right) \\
& =\delta_{1}+\delta_{2}+\delta_{3}+\delta_{4},
\end{aligned}
$$

where

$$
\begin{gathered}
\delta_{1}=\operatorname{Pr}\left[T_{\text {pre- } 1}\right]-\operatorname{Pr}\left[T_{\text {pre- } 2}\right] \\
\delta_{2}=\sum_{\kappa=1}^{Q+1}\left(\left(\operatorname{Pr}\left[T_{\kappa-1}\right]-\operatorname{Pr}\left[T_{\kappa .1}\right]\right)+\left(\operatorname{Pr}\left[T_{\kappa .6}\right]-\operatorname{Pr}\left[T_{\kappa}\right]\right)\right) \\
\delta_{3}=\sum_{\kappa=1}^{Q+1}\left(\left(\operatorname{Pr}\left[T_{\kappa .2}\right]-\operatorname{Pr}\left[T_{\kappa .3}\right]\right)+\left(\operatorname{Pr}\left[T_{\kappa .4}\right]-\operatorname{Pr}\left[T_{\kappa .5}\right]\right)\right) \\
\delta_{4}=\sum_{\kappa=1}^{Q+1}\left(\operatorname{Pr}\left[T_{\kappa .3}\right]-\operatorname{Pr}\left[T_{\kappa .4}\right]\right) .
\end{gathered}
$$

Remark that we have set Game pre-3 equals to Game 0, and SubGame $\kappa .7$ equals to Game $\kappa$. Here, owing to the source ciphertext hiding property, $\operatorname{Pr}\left[T_{\text {pre-0 }}\right]=\operatorname{Pr}\left[T_{\text {pre-1 }}\right]$ holds. Moreover, since neighbor games are identical when we modify the way to compute values of $\mathbf{P}, \widehat{\mathbf{P}}$, and $\widetilde{\mathbf{P}}$ from the public (resp. private) evaluation algorithms to the private (resp. public) evaluation algorithms, $\operatorname{Pr}\left[T_{\text {pre- } 2}\right]=\operatorname{Pr}\left[T_{\text {pre- } 3}\right], \operatorname{Pr}\left[T_{\kappa .1}\right]=\operatorname{Pr}\left[T_{\kappa .2}\right]$, and $\operatorname{Pr}\left[T_{\kappa .5}\right]=\operatorname{Pr}\left[T_{\kappa .6}\right]$ hold. From now, we evaluate the quantities $\delta_{1}, \delta_{2}, \delta_{3}$ and $\delta_{4}$ above. Briefly, $\delta_{1}$ is a quantity that relates critical queries, $\delta_{2}$ is a quantity that relates whether the first component of the ciphertext is chosen from $X^{\prime} \backslash L$ or $L, \delta_{3}$ is a quantity that relates whether the rejection rule is original one or enhanced one, and $\delta_{4}$ is a quantity that relates whether the plaintext of source (resp. challenge) ciphertext is original or random.

Evaluation of $\delta_{1}$ : We note that $\delta_{1}=0$ for the cases of Assumption I and Assumption A. On the other hand, for the case of Assumption U, we divide the game-hopping from Game pre-1 to Game pre-2 by introducing the following subdivision (see Table 5 for a summary): 
Table 5: Summary of differences of games from Game pre-1 to Game pre-2; see the main text for details

\begin{tabular}{|c|c|c|c|c|c|}
\hline Game & pre- $2.0=$ pre- 1 & \begin{tabular}{l|l}
$\ldots$ & pre-2. $(\kappa-1)$
\end{tabular} & pre-2. $\kappa$ & $\ldots$ & pre- $2 . Q=$ pre- 2 \\
\hline $\begin{array}{c}\text { Reply to } \\
\text { first evaluation } \\
\text { query }\end{array}$ & ordinary & \multicolumn{4}{|c|}{$\begin{array}{l}\text { rejected } \\
\text { if critical }\end{array}$} \\
\hline$\vdots$ & & & & & \\
\hline $\begin{array}{c}\text { Reply to } \\
\kappa \text {-th evaluation } \\
\text { query }\end{array}$ & \multicolumn{2}{|c|}{ ordinary } & \multicolumn{3}{|c|}{$\begin{array}{l}\text { rejected } \\
\text { if critical }\end{array}$} \\
\hline$\vdots$ & & & & & \\
\hline $\begin{array}{c}\text { Reply to } \\
Q \text {-th evaluation } \\
\text { query }\end{array}$ & \multicolumn{3}{|c|}{ ordinary } & & $\begin{array}{l}\text { rejected } \\
\text { if critical }\end{array}$ \\
\hline
\end{tabular}

Why negligible

difference

SubGame pre-2. $\kappa(0 \leq \kappa \leq Q)$ : In the game, we modify Game pre-1 in such a way that the challenger rejects any of the first $\kappa$ evaluation queries that is a critical query. Note that SubGame pre-2.0 and SubGame pre-2.Q are the same as Game pre-1 and Game pre-2, respectively. Note also that the complexity of the game is polynomial, by the efficiency of deciding whether a given integer is a critical integer or not (see Assumption U).

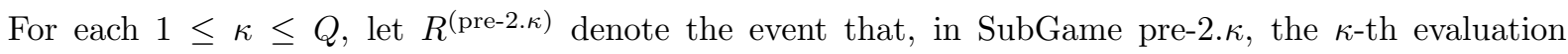
query (exists and) is a critical query. Since SubGame pre-2. $(\kappa-1)$ and SubGame pre-2. $\kappa$ are identical unless the $\kappa$-th evaluation query is a critical query, we have $\left|\operatorname{Pr}\left[T_{\text {pre-2.( }(\kappa-1)}\right]-\operatorname{Pr}\left[T_{\text {pre-2. }}\right]\right| \leq \operatorname{Pr}\left[R^{(\text {pre-2. } \kappa)}\right]$,

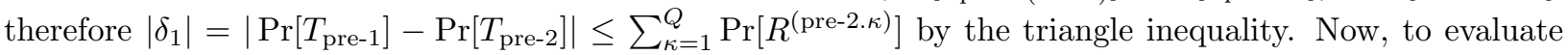
the right-hand side, we introduce the following auxiliary adversary $\mathcal{A}_{1}$ finding a critical integer:

- Given input $1^{\ell}$ for $\mathcal{A}_{1}$, first $\mathcal{A}_{1}$ chooses a game uniformly at random from the $Q$ Games pre-2.1 to

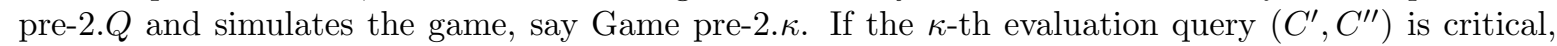
then $\mathcal{A}_{1}$ outputs $\lambda_{\mathcal{D}}\left(\iota_{\mathcal{D}}\left(C^{\prime}\right)\right)+\lambda_{\mathcal{D}}\left(\iota_{\mathcal{D}}\left(C^{\prime \prime}\right)\right)$ which is a critical integer. Otherwise, $\mathcal{A}_{1}$ aborts the game. ${ }^{3}$

By the definition, $\mathcal{A}_{1}$ is PPT, and the advantage $A d v_{\mathcal{A}_{1}}$ of $\mathcal{A}_{1}$ (that is, the probability that $\mathcal{A}_{1}$ output a

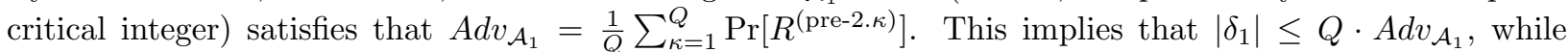
$A d v_{\mathcal{A}_{1}}$ is negligible since finding a critical integer is hard by Assumption U. Hence, $\left|\delta_{1}\right|$ is negligible as well.

Evaluation of $\delta_{2}$ : In order to evaluate the quantity $\delta_{2}$, we introduce the following auxiliary distinguisher $\mathcal{A}_{2}$ for the underlying subset membership problem for $\mathbf{P}$ :

- The distinguisher $\mathcal{A}_{2}$ chooses a game uniformly at random from SubGame $\kappa .1$ with $1 \leq \kappa \leq Q+1$ and SubGame $\kappa .6$ with $1 \leq \kappa \leq Q+1$, and simulates the game by using the given instance $\Lambda$. At the challenge stage of the simulated game, $\mathcal{A}_{2}$ receives $M_{0}^{*}$ and $M_{1}^{*}$ from $\mathcal{A}$. Then:

- When $\kappa \leq Q, \mathcal{A}_{2}$ has received $x^{*(\kappa)} \in L$ or $x^{*(\kappa)} \in X^{\prime} \backslash L$ from the challenger for the subset membership problem. Then $\mathcal{A}_{2}$ generates a challenge ciphertext for $\mathcal{A}$ as usual and sends it to $\mathcal{A}$.

- When $\kappa=Q+1, \mathcal{A}_{2}$ has received $x^{*} \in L$ or $x^{*} \in X^{\prime} \backslash L$ from the challenger for the subset membership problem. Then $\mathcal{A}_{2}$ generates a challenge ciphertext for $\mathcal{A}$ by using the element $x^{*}$ as its first component, and sends it to $\mathcal{A}$.

At the remaining part of the simulated game for the case $\kappa \leq Q, \mathcal{A}_{2}$ generates $\kappa$-th source ciphertext for $\mathcal{A}$ by using the element $x^{*(\kappa)}$ above as its first component. Finally:

\footnotetext{
${ }^{3}$ More precisely, the adversary outputs any object that trivially yields loss of the game.
} 
Table 6: Summary of differences of games from SubGame $\kappa .2$ to SubGame $\kappa .3$; see the main text for details

\begin{tabular}{|c|c|c|c|c|c|c|}
\hline Game & $\kappa .3 .0=\kappa .2$ & $\ldots$ & $\kappa .3 .(\rho-1)$ & $\kappa .3 . \rho$ & $\ldots$ & $\kappa .3 . Q=\kappa .3$ \\
\hline $\begin{array}{l}\text { first query with } \\
\text { irregular input }\end{array}$ & ordinary & \multicolumn{5}{|c|}{ rejected } \\
\hline $\begin{array}{l}\rho \text {-th query with } \\
\text { irregular input }\end{array}$ & \multicolumn{3}{|c|}{ ordinary } & \multicolumn{3}{|c|}{ rejected } \\
\hline $\begin{array}{l}Q \text {-th query with } \\
\text { irregular input }\end{array}$ & \multicolumn{5}{|c|}{ ordinary } & rejected \\
\hline $\begin{array}{c}\text { Why negligible } \\
\text { difference }\end{array}$ & & & $\leftarrow \begin{array}{c}\widehat{\mathbf{P}} \text { and } \\
\text { unive }\end{array}$ & are $\rightarrow$ & & \\
\hline
\end{tabular}

- For the case of SubGame $\kappa .1, \mathcal{A}_{2}$ outputs the opposite bit to the output bit of $\mathcal{A}$; that is, $\mathcal{A}_{2}$ outputs $1-b$ if $\mathcal{A}$ outputs $b$. (Note that the simulated game is identical to Game $(\kappa-1)$ and SubGame $\kappa .1$ if the challenger gave an element of $L$ and of $X^{\prime} \backslash L$, respectively.)

- For the case of SubGame $\kappa .6, \mathcal{A}_{2}$ outputs the output bit of $\mathcal{A}$. (Note that the simulated game is identical to Game $\kappa$ and SubGame $\kappa .6$ if the challenger gave an element of $L$ and of $X^{\prime} \backslash L$, respectively.)

We note that $\mathcal{A}_{2}$ is PPT. By the construction of $\mathcal{A}_{2}$, we have

$$
\operatorname{Pr}\left[1 \leftarrow \mathcal{A}_{2} \mid L \text { is chosen }\right]=\frac{1}{2(Q+1)} \sum_{\kappa=1}^{Q+1}\left(\left(1-\operatorname{Pr}\left[T_{\kappa-1}\right]\right)+\operatorname{Pr}\left[T_{\kappa}\right]\right)
$$

and

$$
\operatorname{Pr}\left[1 \leftarrow \mathcal{A}_{2} \mid X^{\prime} \backslash L \text { is chosen }\right]=\frac{1}{2(Q+1)} \sum_{\kappa=1}^{Q+1}\left(\left(1-\operatorname{Pr}\left[T_{\kappa .1}\right]\right)+\operatorname{Pr}\left[T_{\kappa .6}\right]\right)
$$

therefore

$$
\begin{aligned}
& \operatorname{Pr}\left[1 \leftarrow \mathcal{A}_{2} \mid X^{\prime} \backslash L\right]-\operatorname{Pr}\left[1 \leftarrow \mathcal{A}_{2} \mid L\right] \\
& =\frac{1}{2(Q+1)} \sum_{\kappa=1}^{Q+1}\left(\operatorname{Pr}\left[T_{\ell}^{(\kappa-1)}\right]-\operatorname{Pr}\left[T_{\ell}^{(\kappa)}\right]-\operatorname{Pr}\left[T_{\ell}^{(\kappa \cdot 1)}\right]+\operatorname{Pr}\left[T_{\ell}^{(\kappa .6)}\right]\right)=\frac{\delta_{2}}{2(Q+1)}
\end{aligned}
$$

Hence we have

$$
\left|\delta_{2}\right|=4(Q+1) A d v_{\mathcal{A}_{2}}(\ell),
$$

which is negligible by the assumption that the subset membership problem is hard.

Evaluation of $\delta_{3}$ : From now, we evaluate the quantity $\delta_{3}$. For the purpose, first we divide the game-hopping from SubGame $\kappa .2$ to $\kappa .3$ for each $1 \leq \kappa \leq Q+1$ by introducing the following subdivision (see Table 6 for a summary):

SubSubGame $\kappa .3 . \rho(0 \leq \rho \leq Q)$ : In comparison to SubGame $\kappa .2$, in the game, we replace the original rejection rules with the enhanced rejection rules for the first $\rho$ decryption or evaluation queries. Note that SubSubGame $\kappa .3 .0$ and SubSubGame $\kappa .3 . Q$ are the same as SubGame $\kappa .2$ and SubGame $\kappa .3$, respectively.

Let $R^{(\kappa .3 . \rho)}$ denote the event in SubSubGame $\kappa .3 . \rho$ that the $\rho$-th query (exists and) is rejected by the enhanced rejection rule but is not rejected by the original rejection rule. Then we have $\mid \operatorname{Pr}\left[T_{\kappa .3 .}(\rho-1)\right]-$ 
$\operatorname{Pr}\left[T_{\kappa .3 . \rho}\right] \mid \leq \operatorname{Pr}\left[R^{(\kappa .3 . \rho)}\right]$, therefore $\left|\operatorname{Pr}\left[T_{\kappa .2}\right]-\operatorname{Pr}\left[T_{\kappa .3}\right]\right| \leq \sum_{\rho=1}^{Q} \operatorname{Pr}\left[R^{(\kappa .3 . \rho)}\right]$ by the triangle inequality. Now, to evaluate the right-hand side, we prove the following properties.

Claim 1. In SubSubGame $\kappa .3 . \rho$, among the private information on the keys for $\mathbf{P}, \widehat{\mathbf{P}}$, and $\widetilde{\mathbf{P}}$ that are not obtained by using RevHK (which we call non-queried keys), the collection of replies to the first $\rho$ queries depends only on at most one value of each of $H, \widehat{H}$, and $\widetilde{H}$ for irregular inputs. Moreover, the replies to the evaluation queries (that are not rejected) among the first $\rho$ queries are regular ciphertexts, except for the case of $\kappa$-th refreshing query which is one of the $\rho$ queries provided $\kappa \leq Q$. In this exceptional case, let $\mathcal{D}=$ $\left(C_{0}=C^{*}, C_{1}, \ldots, C_{\kappa}\right)$ and $\mathcal{D}^{\prime}=\left(\left(D_{1}^{\prime}, D_{1}^{\prime \prime}\right), \ldots,\left(D_{\kappa}^{\prime}, D_{\kappa}^{\prime \prime}\right)\right)$ be the two dictionaries after the $\kappa$-th refreshing query, let $\bar{C}_{0}{ }^{(\kappa)}, \bar{C}_{1}{ }^{(\kappa)}, \ldots, \bar{C}_{\kappa}{ }^{(\kappa)}$ be the ciphertexts calculated in the $\kappa$-th refreshing process (hence $\bar{C}_{0}{ }^{(\kappa)}$ is the source ciphertext $\bar{C}^{*}(\kappa)=\left(x^{*(\kappa)}, e^{*(\kappa)}, \widehat{\pi}^{*(\kappa)}, \widetilde{\pi}^{*(\kappa)}\right)$ and $\left.\bar{C}_{\kappa}{ }^{(\kappa)}=C_{\kappa}\right)$, and put $\bar{C}_{\kappa^{\prime}}(\kappa)=\left(x_{\kappa^{\prime}}, e_{\kappa^{\prime}}, \widehat{\pi}_{\kappa^{\prime}}, \widetilde{\pi}_{\kappa^{\prime}}\right)$ for each $\kappa^{\prime}=0,1, \ldots, \kappa$. Then for each $\kappa^{\prime}$, we have:

- $x_{\kappa^{\prime}}$ is the sum of $\lambda_{\mathcal{D}}\left(\kappa^{\prime}\right) \cdot x^{*(\kappa)}$, an integer linear combination of elements of $L$ independent of $x^{*(\kappa)}$, and an integer linear combination of the first components of ciphertexts $D_{i}^{\prime}$ and $D_{i}^{\prime \prime}$ (i.e., those $D_{i}^{\prime}$ and $D_{i}^{\prime \prime}$ are not indices) listed in $\mathcal{D}^{\prime}$ with $1 \leq i \leq \kappa^{\prime}$. Hence, $x_{\kappa^{\prime}}-\lambda_{\mathcal{D}}\left(\kappa^{\prime}\right) \cdot x^{*(\kappa)}$ is an element of $L$ independent of $x^{*(\kappa)}$.

- $e_{\kappa^{\prime}}$ is the sum of $\lambda_{\mathcal{D}}\left(\kappa^{\prime}\right) \cdot e^{*(\kappa)}$, an integer linear combination of elements of the form $H_{k}(\bar{x})$ with $\bar{x} \in L$ being independent of $x^{*(\kappa)}$, and an integer linear combination of the second components of ciphertexts $D_{i}^{\prime}$ and $D_{i}^{\prime \prime}$ listed in $\mathcal{D}^{\prime}$ with $1 \leq i \leq \kappa^{\prime}$.

- For any ciphertext $C=(x, e, \widehat{\pi}, \widetilde{\pi})$, we define $\widehat{\Delta}(C)=\widehat{\pi}-\widehat{H}_{\widehat{k}}(x)$. Then, $\widehat{\Delta}\left(\bar{C}_{\kappa^{\prime}}(\kappa)\right)$ is an integer linear combination of $\widehat{\Delta}\left(D_{i}^{\prime}\right)$ and $\widehat{\Delta}\left(D_{i}^{\prime \prime}\right)$ for ciphertexts $D_{i}^{\prime}$ and $D_{i}^{\prime \prime}$ listed in $\mathcal{D}^{\prime}$ with $1 \leq i \leq \kappa^{\prime}$. Moreover, the calculation of these $\widehat{\Delta}\left(D_{i}^{\prime}\right)$ and $\widehat{\Delta}\left(D_{i}^{\prime \prime}\right)$ from given the $D_{i}^{\prime}$ and $D_{i}^{\prime \prime}$ is independent of the private information on the keys for $\widehat{\mathbf{P}}$.

Proof of Claim 1. We focus on any of the first $\rho$ queries. First, by the enhanced rejection rule, any decryption query with irregular input is always rejected. Note that these queries are not in the dictionary $\mathcal{D}$ owing to the restriction of the decryption oracle. Secondly, the replies to any decryption query with regular input and to any evaluation query with regular input that are not in $\mathcal{D}$ only depends on public values owing to the projective hashes for regular inputs, and therefore the private information on the non-queried keys does not increase by the reply. Hence, it suffices to consider the case of an evaluation query, say $\left(C^{\prime}, C^{\prime \prime}\right)$ with $C^{\prime}=\left(x^{\prime}, e^{\prime}, \widehat{\pi}^{\prime}, \widetilde{\pi}^{\prime}\right)$ and $C^{\prime \prime}=\left(x^{\prime \prime}, e^{\prime \prime}, \widehat{\pi}^{\prime \prime}, \widetilde{\pi}^{\prime \prime}\right)$, where at least one of $C^{\prime}$ and $C^{\prime \prime}$ is either irregular or in $\mathcal{D}$. Moreover, by the enhanced rejection rule, the query is rejected if at least one of $C^{\prime}$ and $C^{\prime \prime}$ is irregular and not in $\mathcal{D}$. Hence, it suffices to consider the case that at least one of $C^{\prime}$ and $C^{\prime \prime}$ is in $\mathcal{D}$ and the other input is either in $\mathcal{D}$ or regular. We also assume that the latter input is $\widetilde{H}$-consistent (which can be now checked without private information on the keys), RevHK has not been queried, and the query is not a critical query (for the case of Assumption U; see Game pre-2), since otherwise the query is rejected. These conditions imply that the query is a (say, $\kappa^{\dagger}$-th) refreshing query.

Let $\mathcal{D}=\left(C_{0}=C^{*}, C_{1}, \ldots, C_{\kappa^{\dagger}-1}\right)$ and $\mathcal{D}^{\prime}=\left(\left(D_{1}^{\prime}, D_{1}^{\prime \prime}\right), \ldots,\left(D_{\kappa^{\dagger}-1}^{\prime}, D_{\kappa^{\dagger}-1}^{\prime \prime}\right)\right)$ be the two dictionaries before this query. Let $C_{\kappa^{\dagger}}$ be the reply to the query, which is added to $\mathcal{D}$ by this query, and let $\left(D_{\kappa^{\dagger}}^{\prime}, D_{\kappa^{\dagger}}^{\prime \prime}\right)$ be the pair added to $\mathcal{D}^{\prime}$ by this query. Let $\bar{C}_{0}\left(\kappa^{\dagger}\right), \bar{C}_{1}\left(\kappa^{\dagger}\right), \ldots, \bar{C}_{\kappa^{\dagger}}{ }^{\left(\kappa^{\dagger}\right)}$ be the ciphertexts calculated in this refreshing process, hence $\bar{C}_{0}{ }^{\left(\kappa^{\dagger}\right)}=\bar{C}^{*}\left(\kappa^{\dagger}\right)$ and $\left.\bar{C}_{\kappa^{\dagger}} \kappa^{\dagger}\right)=C_{\kappa^{\dagger}}$. We note that, any object $D_{i}^{\prime}$ or $D_{i}^{\prime \prime}$, $1 \leq i \leq \kappa^{\dagger}-1$, in $\mathcal{D}^{\prime}$ which is a ciphertext (i.e., not an index) is an input ciphertext (which was not in $\mathcal{D}$ ) for some previous evaluation query which was not rejected, therefore it is a regular ciphertext by the enhanced rejection rule. On the other hand, since any of $C^{\prime}$ and $C^{\prime \prime}$ that is not in $\mathcal{D}$ is regular as discussed above, it follows that any of $D_{\kappa^{\dagger}}^{\prime}$ and $D_{\kappa^{\dagger}}^{\prime \prime}$ that is a ciphertext is also regular.

From now, we first consider the case $\kappa^{\dagger} \neq \kappa$. We show that all the ciphertexts appearing in this refreshing process are regular, therefore $C_{\kappa^{\dagger}}$ is also regular and the calculation of $C_{\kappa^{\dagger}}$ does not use the private information on the keys. The claim for $\bar{C}_{0}{ }^{\left(\kappa^{\dagger}\right)}=\bar{C}^{*}\left(\kappa^{\dagger}\right)$ follows from the construction $\bar{C}^{*}\left(\kappa^{\dagger}\right) \leftarrow \operatorname{Enc} c^{\prime \prime}\left(p k, M_{\beta}^{*}\right)$ (recall that $\left.\kappa^{\dagger} \neq \kappa\right)$. On the other hand, for $1 \leq i \leq \kappa^{\dagger}, \bar{C}_{i}\left(\kappa^{\dagger}\right)$ is the output of Eval" with inputs being 
either a ciphertext listed in $\mathcal{D}^{\prime}$ or the ciphertext $\bar{C}_{j}{ }^{\left(\kappa^{\dagger}\right)}$ for some $0 \leq j<i$. By the induction on $i$ and the argument in the previous paragraph, both of the two input ciphertexts for the Eval" are regular, therefore $\bar{C}_{i}{ }^{\left(\kappa^{\dagger}\right)}$ is also regular and the calculation of $\bar{C}_{i}{ }^{\left(\kappa^{\dagger}\right)}$ does not use the private information on the keys. Hence, the claim holds for the present case $\kappa^{\dagger} \neq \kappa$.

Finally, we consider the case $\kappa^{\dagger}=\kappa$. To show the properties in the statement, we use induction on $\kappa^{\prime}=0,1, \ldots, \kappa$. For the case $\kappa^{\prime}=0$, since $\lambda_{\mathcal{D}}(0)=1$ and $\bar{C}_{0}{ }^{(\kappa)}=\bar{C}^{*}(\kappa)$, the claim follows from the construction of $\bar{C}^{*}(\kappa)$ in the $\kappa$-th refreshing process. We suppose that $\kappa^{\prime}>0$. We divide the proof into the following three cases:

- Suppose that $D_{\kappa^{\prime}}^{\prime}$ is an index $h^{\prime} \in\left\{0,1, \ldots, \kappa^{\prime}-1\right\}$ and $D_{\kappa^{\prime}}^{\prime \prime}$ is an index $h^{\prime \prime} \in\left\{0,1, \ldots, \kappa^{\prime}-1\right\}$. In this case, by the definition of Eval', for some $\bar{x} \stackrel{\$}{\leftarrow} L$ and $\bar{e}=H_{k}(\bar{x})$ (computed without private information on the key), we have $x_{\kappa^{\prime}}=x_{h^{\prime}}+x_{h^{\prime \prime}}+\bar{x}, e_{\kappa^{\prime}}=e_{h^{\prime}}+e_{h^{\prime \prime}}+\bar{e}$, and

$$
\begin{aligned}
\widehat{\Delta}\left(\bar{C}_{\kappa^{\prime}}(\kappa)\right) & =\widehat{\pi}_{\kappa^{\prime}}-\widehat{H}_{\widehat{k}}\left(x_{\kappa^{\prime}}\right) \\
& =\widehat{\pi}_{h^{\prime}}+\widehat{\pi}_{h^{\prime \prime}}+\widehat{H}_{\widehat{k}}(\bar{x})-\widehat{H}_{\widehat{k}}\left(x_{h^{\prime}}\right)-\widehat{H}_{\widehat{k}}\left(x_{h^{\prime \prime}}\right)-\widehat{H}_{\widehat{k}}(\bar{x}) \\
& =\widehat{\Delta}\left(\bar{C}_{h^{\prime}}(\kappa)\right)+\widehat{\Delta}\left(\bar{C}_{h^{\prime \prime}}(\kappa)\right)
\end{aligned}
$$

since $\widehat{\mathbf{P}}$ is homomorphic. Then, since $\lambda_{\mathcal{D}}\left(\kappa^{\prime}\right)=\lambda_{\mathcal{D}}\left(h^{\prime}\right)+\lambda_{\mathcal{D}}\left(h^{\prime \prime}\right)$ by definition, the induction hypothesis for $\bar{C}_{h^{\prime}}{ }^{(\kappa)}$ and $\bar{C}_{h^{\prime \prime}}{ }^{(\kappa)}$ implies that the claim here also holds for the $\bar{C}_{\kappa^{\prime}}(\kappa)$.

- Suppose that $D_{\kappa^{\prime}}^{\prime}$ is an index $h^{\prime} \in\left\{0,1, \ldots, \kappa^{\prime}-1\right\}$ and $D_{\kappa^{\prime}}^{\prime \prime}=\left(x^{\dagger}, e^{\dagger}, \widehat{\pi}^{\dagger}, \tilde{\pi}^{\dagger}\right)$ is a ciphertext. In this case, by the definition of Eval', for some $\bar{x} \stackrel{\$}{\leftarrow} L$ and $\bar{e}=H_{k}(\bar{x})$ (computed without private information on the key), we have $x_{\kappa^{\prime}}=x_{h^{\prime}}+x^{\dagger}+\bar{x}, e_{\kappa^{\prime}}=e_{h^{\prime}}+e^{\dagger}+\bar{e}$, and

$$
\begin{aligned}
\widehat{\Delta}\left(\bar{C}_{\kappa^{\prime}}{ }^{(\kappa)}\right) & =\widehat{\pi}_{\kappa^{\prime}}-\widehat{H}_{\widehat{k}}\left(x_{\kappa^{\prime}}\right) \\
& =\widehat{\pi}_{h^{\prime}}+\widehat{\pi}^{\dagger}+\widehat{H}_{\widehat{k}}(\bar{x})-\widehat{H}_{\widehat{k}}\left(x_{h^{\prime}}\right)-\widehat{H}_{\widehat{k}}\left(x^{\dagger}\right)-\widehat{H}_{\widehat{k}}(\bar{x}) \\
& =\widehat{\Delta}\left(\bar{C}_{h^{\prime}}(\kappa)\right)+\widehat{\Delta}\left(D_{\kappa^{\prime}}^{\prime \prime}\right)
\end{aligned}
$$

since $\widehat{\mathbf{P}}$ is homomorphic. Then, since $\lambda_{\mathcal{D}}\left(\kappa^{\prime}\right)=\lambda_{\mathcal{D}}\left(h^{\prime}\right)$ by definition, the induction hypothesis for $\bar{C}_{h^{\prime}}{ }^{(\kappa)}$ implies that the claim here also holds for the $\bar{C}_{\kappa^{\prime}}(\kappa)$.

- Suppose that $D_{\kappa^{\prime}}^{\prime}$ is a ciphertext and $D_{\kappa^{\prime}}^{\prime \prime}$ is an index. In the case, the symmetry implies that the claim holds by the same argument as the previous case.

Hence the claim holds for any $\kappa^{\prime}=0,1, \ldots, \kappa$; in particular, $C_{\kappa}=\bar{C}_{\kappa}^{(\kappa)}$ is of the form as in the statement, where only a single value of $H$ with irregular input is used to compute $e^{*(\kappa)}$, only a single value of $\widehat{H}$ with irregular input is used to compute $\widehat{\pi}_{\kappa}=\widehat{\Delta}\left(C_{\kappa}\right)+\widehat{H}_{\widehat{k}}\left(x_{\kappa}\right)$, and only a single value of $\widetilde{H}$ with irregular input is used to compute $\widetilde{\pi}_{\kappa}$. This completes the proof of Claim 1 .

Claim 2. For the case of Assumption U, in SubSubGame $\kappa .3 . \rho$ with $\kappa \leq Q$, suppose that the $\kappa$-th refreshing query is $\rho$-th or earlier query and $C_{\kappa}=\left(x_{\kappa}, e_{\kappa}, \widehat{\pi}_{\kappa}, \widetilde{\pi}_{\kappa}\right)$ is its reply. If $\lambda_{\mathcal{D}}(\kappa)$ is not a multiple of $o(\Lambda)$ (see Definition 4.3 for the definition of $o(\Lambda)$ ), then both $\lambda_{\mathcal{D}}(\kappa) \cdot x^{*(\kappa)}$ and $x_{\kappa}$ are uniformly random over $X^{\prime} \backslash L$; otherwise, both $\lambda_{\mathcal{D}}(\kappa) \cdot x^{*(\kappa)}$ and $x_{\kappa}$ are always in $L$.

Proof of Claim 2. First, we consider the case that $\lambda_{\mathcal{D}}(\kappa)$ is a multiple of $o(\Lambda)$. By the definition of $o(\Lambda)$, $\lambda_{\mathcal{D}}(\kappa)$ is a multiple of the order of $x^{*(\kappa)}$ in the quotient group $X / L$, therefore we have $\lambda_{\mathcal{D}}(\kappa) \cdot x^{*(\kappa)} \in L$. Since $x_{\kappa}-\lambda_{\mathcal{D}}(\kappa) \cdot x^{*(\kappa)} \in L$ by Claim 1 , it follows that $x_{\kappa} \in L$, as desired.

Secondly, we consider the other case that $\lambda_{\mathcal{D}}(\kappa)$ is not a multiple of $o(\Lambda)$. Since any critical query is rejected (see Game pre-2), $\lambda_{\mathcal{D}}(h)$ is not a critical integer for any index $h$ by induction on $h$. By the definition of critical integers, it follows that $\lambda_{\mathcal{D}}(\kappa)$ is coprime to $|X|$. Therefore, there is an integer $\lambda^{\prime}$ satisfying that $\lambda_{\mathcal{D}}(\kappa) \lambda^{\prime} \equiv 1(\bmod |X|)$, which is also coprime to $|X|$. This relation implies that the multiplications by 
$\lambda_{\mathcal{D}}(\kappa)$ and by $\lambda^{\prime}$ define two mappings $X \rightarrow X$ which are inverses of each other. Moreover, each of the mappings maps $L$ to $L$ since $L$ is a subgroup of $X$, while by Assumtion U, it maps $X^{\prime} \backslash L$ to $X^{\prime}$. This implies that the multiplication by $\lambda_{\mathcal{D}}(\kappa)$ defines a bijection $X^{\prime} \backslash L \rightarrow X^{\prime} \backslash L$, therefore $\lambda_{\mathcal{D}}(h) \cdot x^{*(\kappa)}$ is uniformly random over $X^{\prime} \backslash L$ as well as $x^{*(\kappa)}$. On the other hand, by Assumption $\mathrm{U}$, for any $y \in L$, the addition by $y$ defines a bijection $X^{\prime} \backslash L \rightarrow X^{\prime} \backslash L$ ( since $L$ is a subgroup of $X$ ). Since $x_{\kappa}-\lambda_{\mathcal{D}}(\kappa) \cdot x^{*(\kappa)}$ is an element of $L$ independent of $x^{*(\kappa)}$ by Claim 1 , it follows that $x_{\kappa}$ is also uniformly random over $X^{\prime} \backslash L$, as desired. This completes the proof of Claim 2 .

Before evaluating the quantities $\operatorname{Pr}\left[R^{(\kappa .3 . \rho)}\right]$, we also introduce subdivision of the game-hopping from SubGame $\kappa .4$ to SubGame $\kappa .5$ for $1 \leq \kappa \leq Q+1$ in a similar manner:

SubSubGame $\kappa .5 . \rho(0 \leq \rho \leq Q)$ : In comparison to SubGame $\kappa .4$, in the game, we replace the enhanced rejection rules for the $(Q+1-\rho)$-th or later queries with the original rejection rules. Note that SubSubGame $\kappa .5 . Q$ and SubSubGame $\kappa .5 .0$ are the same as SubGame $\kappa .4$ and SubGame $\kappa .5$, respectively.

Now we note that each SubSubGame א.5. $\rho$ satisfies properties similar to Claim 1 and Claim 2 above, where the $(Q+1-\rho)$-th query plays the role of the $\rho$-th query in the original Claim 1 and Claim 2.

Let $R^{(\kappa .5 . \rho)}$ denote the event in SubSubGame $\kappa .5 . \rho$ that the $(Q+1-\rho)$-th query (exists and) is rejected by the enhanced rejection rule but is not rejected by the original rejection rule. Then an argument similar to the case of SubSubGame $\kappa .3 . \rho$ implies that $\left|\operatorname{Pr}\left[T_{\kappa .4}\right]-\operatorname{Pr}\left[T_{\kappa .5}\right]\right| \leq \sum_{\rho=1}^{Q} \operatorname{Pr}\left[R^{(\kappa .5 . \rho)}\right]$.

In order to evaluate $\operatorname{Pr}\left[R^{(\kappa .3 . \rho)}\right]$ and $\operatorname{Pr}\left[R^{(\kappa .5 . \rho)}\right]$, we introduce further the following events:

- We define $R_{1}^{(\kappa .3 . \rho)}$ to be the event in SubSubGame $\kappa .3 . \rho$ that the $\rho$-th query is a decryption query with $\widehat{H}$-forging input $C$ and is in the find stage, and RevHK has been queried before this query. In a similar manner, we also define the event $R_{1}^{(\kappa .5 . \rho)}$, where we focus on the $(Q+1-\rho)$-th query instead of the $\rho$-th query.

- We define $R_{2}^{(\kappa .3 . \rho)}$ (respectively, $R_{3}^{(\kappa .3 . \rho)}$ ) to be the event in SubSubGame $\kappa .3 . \rho$ that the $\rho$-th query is a decryption query with input $C$ (respectively, an evaluation query $\left.\left(C^{\prime}, C^{\prime \prime}\right)\right), C$ is $\widetilde{H}$-forging and $C \notin \mathcal{D}$ (respectively, $C^{\prime}$ is $\widetilde{H}$-forging and $C^{\prime} \notin \mathcal{D}$ ), RevHK has not been queried before this query, and:

- In the case $\kappa \leq Q$, either this query is the $\kappa$-th refreshing query or before the $\kappa$-th refreshing query, or this query is after the $\kappa$-th refreshing query and the reply to the $\kappa$-th refreshing query is a regular ciphertext.

- In the case $\kappa=Q+1$, this query is in the find stage.

We define the event $R_{4}^{(\kappa .3 . \rho)}$ to be the same as $R_{3}^{(\kappa .3 . \rho)}$ except that we focus on $C^{\prime \prime}$ instead of $C^{\prime}$. In a similar manner, we also define the events $R_{2}^{(\kappa .5 . \rho)}, R_{3}^{(\kappa .5 . \rho)}$ and $R_{4}^{(\kappa .5 . \rho)}$, where we focus on the $(Q+1-\rho)$-th query instead of the $\rho$-th query.

- We define $R_{5}^{(\kappa .3 . \rho)}$ (respectively, $R_{6}^{(\kappa .3 . \rho)}$ ) to be the event in SubSubGame $\kappa .3 . \rho$ that the $\rho$-th query is a decryption query with input $C$ (respectively, an evaluation query $\left.\left(C^{\prime}, C^{\prime \prime}\right)\right), C$ is $\widetilde{H}$-forging and $C \notin \mathcal{D}$ (respectively, $C^{\prime}$ is $\widetilde{H}$-forging and $C^{\prime} \notin \mathcal{D}$ ), RevHK has not been queried before this query, and:

- In the case $\kappa \leq Q$, this query is after the $\kappa$-th refreshing query and the reply to the $\kappa$-th refreshing query is an irregular ciphertext.

- In the case $\kappa=Q+1$, this query is in the guess stage.

We define the event $R_{7}^{(\kappa .3 . \rho)}$ to be the same as $R_{6}^{(\kappa .3 . \rho)}$ except that we focus on $C^{\prime \prime}$ instead of $C^{\prime}$. In a similar manner, we also define the events $R_{5}^{(\kappa .5 . \rho)}, R_{6}^{(\kappa .5 . \rho)}$ and $R_{7}^{(\kappa .5 . \rho)}$, where we focus on the $(Q+1-\rho)$-th query instead of the $\rho$-th query. 
By the definitions of the events, we have $\operatorname{Pr}\left[R^{(\kappa .3 . \rho)}\right] \leq \sum_{i=1}^{7} \operatorname{Pr}\left[R_{i}^{(\kappa .3 . \rho)}\right]$, and a similar inequality holds for $R^{(\kappa .5 . \rho)}$. Therefore, we have

$$
\left|\delta_{3}\right| \leq \sum_{i=1}^{7} \sum_{\rho=1}^{Q} \sum_{\kappa=1}^{Q+1}\left(\operatorname{Pr}\left[R_{i}^{(\kappa .3 . \rho)}\right]+\operatorname{Pr}\left[R_{i}^{(\kappa .5 . \rho)}\right]\right) .
$$

We evaluate the quantities in the right-hand side of the inequality. Here, we put $\bar{\rho}=\rho$ for the case of events $R^{(\kappa .3 . \rho)}$, and $\bar{\rho}=Q+1-\rho$ for the case of events $R^{(\kappa .5 . \rho)}$.

For the events $R_{1}^{(\cdot)}$, Claim 1 implies that the private information on $\widehat{k}$ is not used in the game before the $\bar{\rho}$-th query, therefore the universal 1 property of $\widehat{\mathbf{P}}$ implies that the adversary can generate the $\widehat{H}$-forging input for the query with only negligible probability common to all $\kappa$ and $\rho$. Hence, the $\operatorname{sum}$ of $\operatorname{Pr}\left[R_{1}^{(\kappa .3 . \rho)}\right]$ and $\operatorname{Pr}\left[R_{1}^{(\kappa .5 . \rho)}\right]$ over all $\kappa$ and $\rho$ is negligible.

For the case of Assumption I, a similar argument based on Claim 1 implies that the sum of $\operatorname{Pr}\left[R_{i}^{(\kappa .3 . \rho)}\right]$ and $\operatorname{Pr}\left[R_{i}^{(\kappa .5 . \rho)}\right]$ over all $i \in\{2,3,4\}, \kappa$, and $\rho$ is negligible owing to the universal 1 property of $\widetilde{\mathbf{P}}$, since the private information on $\widetilde{k}$ is not used in the game before the $\bar{\rho}$-th query. Similarly, Claim 1 implies that the behavior of the game before the $\bar{\rho}$-th query depends only on at most one value of $\widetilde{\mathbf{P}}$ for irregular input among private information on the non-queried keys, therefore the sum of $\operatorname{Pr}\left[R_{i}^{(\kappa .3 . \rho)}\right]$ and $\operatorname{Pr}\left[R_{i}^{(\kappa .5 . \rho)}\right]$ over all $i \in\{5,6,7\}, \kappa$, and $\rho$ is negligible owing to the universal ${ }_{2}$ property of $\widetilde{\mathbf{P}}$. Summarizing, $\left|\delta_{3}\right|$ is negligible for the case of Assumption I.

From now, we consider the other cases of Assumptions A and U. We reduce the evaluation of $\operatorname{Pr}\left[R_{i}^{(\kappa .3 . \rho)}\right]$ and $\operatorname{Pr}\left[R_{i}^{(\kappa .5 . \rho)}\right]$ for $2 \leq i \leq 7$ to evaluation of the advantages of the following two adversaries $\mathcal{A}_{3,1}$ and $\mathcal{A}_{3,2}$ for the security game of the first-adaptive or first-uniform computationally universal 2 property for $\widetilde{\mathbf{P}}$. Here $\mathcal{A}_{3,1}$ corresponds to the events with $2 \leq i \leq 4$, while $\mathcal{A}_{3,2}$ corresponds to the events with $5 \leq i \leq 7$. The descriptions of these adversaries are as follows:

Adversaries $\mathcal{A}_{3,1}$ and $\mathcal{A}_{3,2}$ : First, the adversary generates $(k, s) \leftarrow$ HPS.param $\left(1^{\ell}, \Lambda\right)$ and $(\widehat{k}, \widehat{s}) \leftarrow$ $\widehat{\text { HPS.param }}\left(1^{\ell}, \Lambda\right)$. Remark that $\mathcal{A}_{3,1}$ and $\mathcal{A}_{3,2}$ need to use the private evaluation algorithms of $\mathbf{P}$ and $\widehat{\mathbf{P}}$ for regular inputs by using $k$ and $\widehat{k}$. Again, the projective property of the projective hash family implies that the computed value is not changed by the modification, still the simulation works well. Secondly, the adversary chooses a game from SubSubGame $\kappa .3 . \rho$ (we set $\bar{\rho}=\rho$ in this case) and SubSubGame $\kappa .5 . \rho$ (we set $\bar{\rho}=Q+1-\rho$ in this case) for $1 \leq \kappa \leq Q+1$ and $1 \leq \rho \leq Q$, which we call the internal game in the following, and chooses one of the three modes dec, eval ${ }_{1}$, and eval ${ }_{2}$. Then the adversary simulates the internal game until the $\bar{\rho}$-th query. Here, the adversary aborts when any condition for the corresponding event $R_{i}^{(\cdot)}$ becomes unable to be satisfied. For example, in the case $\kappa=Q+1, \mathcal{A}_{3,1}$ aborts if the find stage of the internal game ends before the $\bar{\rho}$-th query (hence $\mathcal{A}_{3,1}$ does not need to compute the challenge ciphertext).

In the case $\kappa \leq Q$, the adversary deals with the $\kappa$-th refreshing query in the following manner:

- First, $\mathcal{A}_{3,1}$ calculates the first three components $\left(x_{\kappa}, e_{\kappa}, \widehat{\pi}_{\kappa}\right)$ of the resulting ciphertext at the refreshing process, where the first component of the source ciphertext is chosen from $X^{\prime} \backslash L$ uniformly at random. ${ }^{4}$ Then $\mathcal{A}_{3,1}$ queries $\left(x_{\kappa}, e_{\kappa}, \widehat{\pi}_{\kappa}\right)$ to Hash, and aborts if Hash replies $\perp$ (this corresponds to the condition of the event $R_{i}^{(\cdot)}$ with $2 \leq i \leq 4$ that the reply to the $\kappa$-th refreshing query must be regular). Otherwise, $\mathcal{A}_{3,1}$ receives $\widetilde{\pi}_{\kappa}$ from Hash and replies $\left(x_{\kappa}, e_{\kappa}, \widehat{\pi}_{\kappa}, \widetilde{\pi}_{\kappa}\right)$ to the refreshing query.

- First, $\mathcal{A}_{3,2}$ chooses the first component $x^{*(\kappa)}$ of the source ciphertext uniformly at random from $X^{\prime} \backslash L$, and compute the first three components $\left(x_{\kappa}, e_{\kappa}, \widehat{\pi}_{\kappa}\right)$ of the result of the refreshing process according to the formula shown in Claim 1 . Then $\mathcal{A}_{3,2}$ queries $\left(x_{\kappa}, e_{\kappa}, \widehat{\pi}_{\kappa}\right)$ to Hash, and aborts if Hash replies an element other than $\perp$ (this corresponds to the condition of the event $R_{i}^{(\cdot)}$ with $5 \leq i \leq 7$ that the reply to the $\kappa$-th refreshing query must be irregular). Otherwise:

\footnotetext{
${ }^{4}$ Note that this process does not require the key for $\widetilde{\mathbf{P}}$; since all the ciphertexts appearing during the refreshing process are guaranteed to be $\widetilde{H}$-consistent, and the consistency checks for the fourth components can be omitted.
} 
- For the case of Assumption A, $\mathcal{A}_{3,2}$ submits $\left(x_{\kappa}, e_{\kappa}, \widehat{\pi}_{\kappa}\right)$ to the challenger and receives $\widetilde{\pi}_{\kappa}$. Then $\mathcal{A}_{3,2}$ replies $\left(x_{\kappa}, e_{\kappa}, \widehat{\pi}_{\kappa}, \tilde{\pi}_{\kappa}\right)$ to the $\kappa$-th refreshing query.

- For the case of Assumption $\mathrm{U}$, note that $\mathcal{A}_{3,2}$ has given some $\left(x^{* *}, e^{* *}, \widehat{\pi}^{* *}\right)$ and $\widetilde{\pi}^{* *}$ as a part of input. Then $\mathcal{A}_{3,2}$ replies $\left(x^{* *}, e^{* *}, \widehat{\pi}^{* *}, \widetilde{\pi}^{* *}\right)$ to the $\kappa$-th refreshing query.

In the case $\kappa=Q+1, \mathcal{A}_{3,1}$ does not need to deal with the challenge ciphertext in the internal game as mentioned above. On the other hand, $\mathcal{A}_{3,2}$ deals with the challenge ciphertext in the following manner:

- First, $\mathcal{A}_{3,2}$ chooses the first component $x^{*}$ of the challenge ciphertext uniformly at random from $X^{\prime} \backslash L$, and compute the first three components $\left(x^{*}, e^{*}, \widehat{\pi}^{*}\right)$ of the challenge ciphertext. Then:

- For the case of Assumption A, $\mathcal{A}_{3,2}$ submits $\left(x^{*}, e^{*}, \widehat{\pi}^{*}\right)$ to the challenger and receives $\widetilde{\pi}^{*}$. Then $\mathcal{A}_{3,2}$ sets $C^{*}=\left(x^{*}, e^{*}, \widehat{\pi}^{*}, \widetilde{\pi}^{*}\right)$ to be the challenge ciphertext.

- For the case of Assumption U, note that $\mathcal{A}_{3,2}$ has given some $\left(x^{* *}, e^{* *}, \widehat{\pi}^{* *}\right)$ and $\widetilde{\pi}^{* *}$ as a part of input. Then $\mathcal{A}_{3,2}$ sets $\left(x^{* *}, e^{* *}, \widehat{\pi}^{* *}, \widetilde{\pi}^{* *}\right)$ to be the challenge ciphertext.

Finally, we consider the $\bar{\rho}$-th query. Here we note that $\mathcal{A}_{3,1}$ has not submitted anything to the challenger; now, in the case of Assumption A, $\mathcal{A}_{3,1}$ submits $\left(x^{* *}, e^{* *}, \widehat{\pi}^{* *}\right) \in\left(X^{\prime} \backslash L\right) \times \Pi \times \widehat{\Pi}$ chosen uniformly at random and receives $\widetilde{\pi}^{* *}$. Then the adversary $\mathcal{A}_{3,1}$ or $\mathcal{A}_{3,2}$ performs as follows:

- In mode dec, the adversary aborts if this query is not a decryption query. When it is a decryption query with input $C=(x, e, \widehat{\pi}, \widetilde{\pi})$, the adversary aborts if $C \in \mathcal{D}$. Otherwise, the adversary outputs $(x, e, \widehat{\pi})$ and $\tilde{\pi}$.

- In mode eval ${ }_{1}$, the adversary aborts if this query is not an evaluation query. When it is an evaluation query $\left(C^{\prime}, C^{\prime \prime}\right)$ with $C^{\prime}=\left(x^{\prime}, e^{\prime}, \widehat{\pi}^{\prime}, \widetilde{\pi}^{\prime}\right)$, the adversary aborts if $C^{\prime} \in \mathcal{D}$. Otherwise, the adversary outputs $\left(x^{\prime}, e^{\prime}, \widehat{\pi}^{\prime}\right)$ and $\widetilde{\pi}^{\prime}$.

- The case of mode eval 2 is similar to eval ${ }_{1}$, where $C^{\prime \prime}$ plays the role of $C^{\prime}$.

Note that, in the internal game until the $\bar{\rho}$-th query, any other decryption or evaluation query with irregular input ciphertext(s) can be always rejected owing to the enhanced rejection rule, and the oracle Hash enables the adversary to detect the irregular input and, if it is regular, to obtain values of $\widetilde{\mathbf{P}}$ for regular inputs required to respond to the query. (In the case of evaluation query with input ciphertext that is irregular and is in $\mathcal{D}$, even if the query cannot be rejected, the definition of refreshing process ensures that the resulting ciphertext is always regular.) On the other hand, the adversary aborts if RevHK has been queried in the internal game (according to the condition of the corresponding event). This concludes the descriptions of $\mathcal{A}_{3,1}$ and $\mathcal{A}_{3,2}$.

In fact, these adversaries simulate the internal game correctly. The only non-trivial point here is for the reply $\left(x^{* *}, e^{* *}, \widehat{\pi}^{* *}, \widetilde{\pi}^{* *}\right)$ of $\mathcal{A}_{3,2}$ to the $\kappa$-th refreshing query (or to the challenge query, when $\kappa=Q+1$ ) in the case of Assumption U. First, for $\kappa$-th refreshing process in the case $\kappa \leq Q$, since Hash with input $\left(x_{\kappa}, e_{\kappa}, \widehat{\pi}_{\kappa}\right)$ replies $\perp$ (which depends solely on $\lambda_{\mathcal{D}}(\kappa)$ and not on $x^{*(\kappa)}$ by Claim 2), Claim 2 implies that both $\lambda_{\mathcal{D}}(\kappa) \cdot x^{*(\kappa)}$ and $x_{\kappa}$ are uniformly random over $X^{\prime} \backslash L$. Moreover, Claim 1 implies that the private information on the keys for $\mathbf{P}$ and $\widehat{\mathbf{P}}$ are not used during the internal game except for the values $H_{k}\left(\lambda_{\mathcal{D}}(\kappa) \cdot x^{*(\kappa)}\right)=$ $\lambda_{\mathcal{D}}(\kappa) \cdot e^{*(\kappa)}$ and $\widehat{H}_{\widehat{k}}\left(x_{\kappa}\right)$ used in the calculation of $e_{\kappa}$ and $\widehat{\pi}_{\kappa}$. Therefore, since $\Pi^{\prime}=\Pi, \mathbf{P}$ is smooth relative to $\left(X^{\prime}, \Pi^{\prime}\right)$ and $\widehat{\mathbf{P}}$ is smooth relative to $\left(X^{\prime}, \widehat{\Pi}\right)$ by Assumption $\mathrm{U}$, the distributions of $\lambda_{\mathcal{D}}(\kappa) \cdot e^{*(\kappa)}$ and $\widehat{H}_{\widehat{k}}\left(x_{\kappa}\right)$ have negligible statistical distances from the uniform distributions over $\Pi$ and $\widehat{\Pi}$, respectively. Since the differences $e_{\kappa}-\lambda_{\mathcal{D}}(\kappa) \cdot e^{*(\kappa)}$ and $\widehat{\pi}_{\kappa}-\widehat{H}_{\widehat{k}}\left(x_{\kappa}\right)$ are independent of $x^{*(\kappa)}$ by Claim 1, it follows that the distribution of $\left(x_{\kappa}, e_{\kappa}, \widehat{\pi}_{\kappa}\right)$ has negligible statistical distance from the uniform distribution of $\left(x^{* *}, e^{* *}, \widehat{\pi}^{* *}\right)$ over $\left(X^{\prime} \backslash L\right) \times \Pi \times \widehat{\Pi}$. Similarly, for the challenge ciphertext in the case $\kappa=Q+1$, the smoothness of $\mathbf{P}$ and $\widehat{\mathbf{P}}$ as above imply that the distribution of the first three components $\left(x^{*}, e^{*}, \widehat{\pi}^{*}\right)$ of the challenge ciphertext in the internal game has negligible statistical distance from the uniform distribution of $\left(x^{* *}, e^{* *}, \widehat{\pi}^{* *}\right)$ over 
$\left(X^{\prime} \backslash L\right) \times \Pi \times \widehat{\Pi}$. Hence, the simulation of the internal game by the adversaries is correct, with only negligible statistical distance.

By the constructions of the adversaries, $\mathcal{A}_{3, j}(j \in\{1,2\})$ in mode dec (respectively, eval ${ }_{1}$, eval ${ }_{2}$ ) wins the game for $\widetilde{\mathbf{P}}$ if and only if the corresponding event $R_{3 j-1}^{(\cdot)}$ (respectively, $R_{3 j}^{(\cdot)}, R_{3 j+1}^{(\cdot)}$ ) occurs in the internal game unless the uniformly random $\left(x^{* *}, e^{* *}, \widehat{\pi}^{* *}\right)$ contained in the input for $\mathcal{A}_{3,1}$ (in the case of Assumption $U$ ) or chosen by $\mathcal{A}_{3,1}$ (in the case of Assumption A) coincides with the corresponding component of the output of $\mathcal{A}_{3,1}$. Now note that the output of $\mathcal{A}_{3,1}$ is independent of $\left(x^{* *}, e^{* *}, \widehat{\pi}^{* *}\right)$, therefore the exceptional case above occurs with only negligible probability (since otherwise $X^{\prime} \backslash L$ must be not large enough to make the underlying subset membership problem hard). Summarizing, the differences

$$
\left|A d v_{\mathcal{A}_{3,1}}-\frac{1}{6(Q+1) Q} \sum_{i=2}^{4} \sum_{\rho=1}^{Q} \sum_{\kappa=1}^{Q+1}\left(\operatorname{Pr}\left[R_{i}^{(\kappa .3 . \rho)}\right]+\operatorname{Pr}\left[R_{i}^{(\kappa .5 . \rho)}\right]\right)\right|
$$

and

$$
\left|A d v_{\mathcal{A}_{3,2}}-\frac{1}{6(Q+1) Q} \sum_{i=5}^{7} \sum_{\rho=1}^{Q} \sum_{\kappa=1}^{Q+1}\left(\operatorname{Pr}\left[R_{i}^{(\kappa .3 . \rho)}\right]+\operatorname{Pr}\left[R_{i}^{(\kappa .5 . \rho)}\right]\right)\right|
$$

are both negligible. Moreover, we define $\mathcal{A}_{3, j}^{\prime}(j=1,2)$ by modifying $\mathcal{A}_{3, j}$ in such a way that the (possibly inefficient) uniformly random choices of elements of $X^{\prime} \backslash L$ are replaced with statistically close and efficiently samplable distributions over $X$, owing to the assumption that $X^{\prime} \backslash L$ is approximately samplable relative to $X$. Then $\mathcal{A}_{3, j}^{\prime}$ is PPT and $\left|A d v_{\mathcal{A}_{3, j}^{\prime}}-A d v_{\mathcal{A}_{3, j}}\right|$ is negligible, while $A d v_{\mathcal{A}_{3, j}^{\prime}}$ is negligible by the first-adaptive or first-uniform computationally universal 2 property of $\widetilde{\mathbf{P}}$. Hence, the average of probabilities $\operatorname{Pr}\left[R_{i}^{(\kappa .3 . \rho)}\right]$ and $\operatorname{Pr}\left[R_{i}^{(\kappa .5 . \rho)}\right]$ for $2 \leq i \leq 7$, all $\kappa$ and all $\rho$ (polynomially bounded number in total) is negligible, so is the sum of these probabilities. This implies that $\left|\delta_{3}\right|$ is negligible as well.

Evaluation of $\delta_{4}$ : Finally, we evaluate the quantity $\delta_{4}$. By Claim 1 in the evaluation of $\delta_{3}$ above, in SubGame $\kappa .3$ with $\kappa \leq Q$, the private information on the key for $\mathbf{P}$ is not used during the game except the value $H_{k}\left(x^{*(\kappa)}\right)$ for $x^{*(\kappa)} \in X^{\prime} \backslash L$ used in the computation of $e^{*(\kappa)}=M_{\beta}^{*}+H_{k}\left(x^{*(\kappa)}\right)$ in the $\kappa$-th refreshing process. Therefore, by replacing $H_{k}\left(x^{*(\kappa)}\right)$ above with $H_{k}\left(x^{*(\kappa)}\right)+\pi^{\dagger \dagger}$ where $\pi^{\dagger \dagger}$ is chosen uniformly at random from $\Pi^{\prime}$, only negligible statistical distance is induced to the behavior of the game owing to the smoothness of $\mathbf{P}$ relative to $\left(X^{\prime}, \Pi^{\prime}\right)$. Secondly, since the uniformly random $\pi^{\dagger \dagger}$ is independent of $M_{\beta}^{*}$, the element $M_{\beta}^{*}+\pi^{\dagger \dagger}$ is also uniformly random over $\Pi^{\prime}$. Then, owing to the assumption that $\Pi^{\prime}$ is approximately samplable relative to $\Pi$, by replacing the value $M_{\beta}^{*}+\pi^{\dagger \dagger}$ above further with the element $\pi^{\dagger} \in \Pi$ chosen as in the definition of SubGame $\kappa .4$, only negligible statistical distance is induced to the behavior of the game. Now the resulting choice of $e^{*(\kappa)}$ is identical to SubGame $\kappa .4$, therefore $\left|\operatorname{Pr}\left[T_{\kappa .3}\right]-\operatorname{Pr}\left[T_{\kappa .4}\right]\right|$ is negligible for any $\kappa$. The same argument works for the case $\kappa=Q+1$, where the challenge ciphertext plays the role of $\kappa$-th source ciphertext. Summarizing, $\left|\delta_{4}\right|$ is negligible, as desired.

By these results, we have $A d v_{\mathrm{KH}-\mathrm{PKE}, \mathcal{A}}^{\mathrm{KH}}(\ell) \leq\left|\delta_{1}\right|+\left|\delta_{2}\right|+\left|\delta_{3}\right|+\left|\delta_{4}\right|$, while all of $\left|\delta_{1}\right|,\left|\delta_{2}\right|,\left|\delta_{3}\right|$ and $\left|\delta_{4}\right|$ are negligible, therefore the advantage of the adversary $\mathcal{A}$ for our proposed KH-PKE scheme is negligible as well. This completes the proof of Theorem 4.1.

\section{KH-PKE Instantiations of the Generic Construction}

\subsection{Cramer-Shoup Projective Hash Family}

To instantiate the (computationally or information-theoretically) universal ${ }_{2}$ hash proof system $\widetilde{\mathbf{P}}$ in our generic construction of KH-PKE given in Section 4, the construction of hash proof systems proposed by Cramer and Shoup [14, $\$ 7.43$ Theorem 3] based on diverse group systems can be used. Here we recall the definition of the Cramer-Shoup (CS) hash proof system. In fact, we deal with not only the original 
construction based on an injective function as the internal function, but also its variants (already mentioned in [14]) where the internal function is generalized to more various classes of functions.

The construction of the CS projective hash family [14] is as follows. Let $\mathbf{G}=(\mathcal{H}, X, L, \Pi)$ be a diverse group system, and let $\left\{g_{1}, \ldots, g_{d}\right\}$ be a fixed generating set of $L$. Let $E$ be a finite set. Moreover, let $\Gamma: X \times E \rightarrow\{0, \ldots, \widetilde{p}-1\}^{n}$ be a function, where $\widetilde{p}$ is the smallest prime dividing $|X / L|$ (in the original construction, $\Gamma$ is supposed to be an injective function; here we consider more general functions $\Gamma)$. Then the CS projective hash family $\mathbf{H}=(H, K, X \times E, L \times E, \Pi, S, \alpha)$ is constructed as follows:

- We set $K=\mathcal{H}^{n+1}$, and for $\vec{k}=\left(k_{0}, k_{1}, \ldots, k_{n}\right) \in K$ and $(x, e) \in X \times E$, the value of $H$ is defined as follows, where we write $\Gamma(x, e)=\left(\gamma_{1}, \ldots, \gamma_{n}\right)=\left(\gamma_{1}(x, e), \ldots, \gamma_{n}(x, e)\right)$ :

$$
H_{\vec{k}}(x, e)=k_{0}(x)+\sum_{i=1}^{n} \gamma_{i} k_{i}(x)
$$

- We set $S=\Pi^{(n+1) d}$, and for $\vec{k}=\left(k_{0}, k_{1}, \ldots, k_{n}\right) \in K$, the value of $\alpha$ is defined by

$$
\alpha(\vec{k})=\left(k_{0}\left(g_{1}\right), \ldots, k_{0}\left(g_{d}\right), k_{1}\left(g_{1}\right), \ldots, k_{1}\left(g_{d}\right), \ldots, k_{n}\left(g_{1}\right), \ldots, k_{n}\left(g_{d}\right)\right) \text {. }
$$

Now, given a public information $\vec{s}=\alpha(\vec{k})$, an element $(x, e) \in L \times E$ and an expression $x=\sum_{j=1}^{d} \omega_{j} g_{j}$ of $x$ with the generating set $\left\{g_{1}, \ldots, g_{d}\right\}$ of $L$ (which is a witness of $(x, e) \in L \times E$ ), the private evaluation algorithm for the corresponding hash proof system can compute the value of $H$ by

$$
H_{\vec{k}}(x, e)=\sum_{j=1}^{d} \omega_{j} s_{0, j}+\sum_{i=1}^{n} \gamma_{i}(x, e) \sum_{j=1}^{d} \omega_{j} s_{i, j}
$$

where $s_{i, j}=k_{i}\left(g_{j}\right)$ for $i \in\{0,1, \ldots, n\}$ and $j \in\{1, \ldots, d\}$.

The following lemma is the key property for our argument below. We note that essentially the same argument appeared in [14]; here we include the proof for the sake of completeness.

Lemma 5.1. For the CS projective hash family constructed as above, for $\vec{s} \in S,(x, e),\left(x^{*}, e^{*}\right) \in(X \backslash L) \times E$ and $\pi, \pi^{*} \in \Pi$, if $\Gamma(x, e) \neq \Gamma\left(x^{*}, e^{*}\right)$, then we have

$$
\operatorname{Pr}_{\vec{k} \stackrel{\mathbb{\Phi}}{\leftarrow} K}\left[H_{\vec{k}}(x, e)=\pi \wedge H_{\vec{k}}\left(x^{*}, e^{*}\right)=\pi^{*} \wedge \alpha(\vec{k})=\vec{s}\right] \leq \frac{1}{\widetilde{p}} \cdot \operatorname{Pr}_{\vec{k} \underset{\leftarrow}{\leftarrow} K}\left[H_{\vec{k}}\left(x^{*}, e^{*}\right)=\pi^{*} \wedge \alpha(\vec{k})=\vec{s}\right] .
$$

Proof. Since $\Gamma(x, e) \neq \Gamma\left(x^{*}, e^{*}\right)$, by symmetry, we may assume without loss of generality that $\gamma_{n}(x, e) \neq$ $\gamma_{n}\left(x^{*}, e^{*}\right)$. Now the left-hand side of the inequality in the statement is equal to

$$
\sum_{\vec{k} \in K(\vec{s})} \frac{1}{|\mathcal{H}|^{n+1}} \cdot \chi\left[k_{0}(x)+\gamma_{n}(x, e) k_{n}(x)=\bar{\pi} \wedge k_{0}\left(x^{*}\right)+\gamma_{n}\left(x^{*}, e^{*}\right) k_{n}\left(x^{*}\right)=\bar{\pi}^{*}\right]
$$

where $K(\vec{s})$ denotes the set of all $\vec{k} \in K$ satisfying that $\alpha(\vec{k})=\vec{s}$, we put $\bar{\pi}=\pi-\sum_{i=1}^{n-1} \gamma_{i}(x, e) k_{i}(x)$ and $\bar{\pi}^{*}=\pi^{*}-\sum_{i=1}^{n-1} \gamma_{i}\left(x^{*}, e^{*}\right) k_{i}\left(x^{*}\right)$, and $\chi[\cdot]$ denotes the characteristic function that returns 1 if the specified condition is satisfied and returns 0 otherwise. Similarly, the right-hand side of the inequality in the statement is equal to

$$
\frac{1}{\widetilde{p}} \cdot \sum_{\vec{k} \in K(\vec{s})} \frac{1}{|\mathcal{H}|^{n+1}} \cdot \chi\left[k_{0}\left(x^{*}\right)+\gamma_{n}\left(x^{*}, e^{*}\right) k_{n}\left(x^{*}\right)=\bar{\pi}^{*}\right] .
$$

Therefore, it suffices to show that, for any $k_{1}, \ldots, k_{n-1} \in \mathcal{H}$, we have

$$
\begin{array}{r}
\sum_{\left(k_{0}, k_{n}\right) \in K^{\prime}} \chi\left[k_{0}(x)+\gamma_{n}(x, e) k_{n}(x)=\bar{\pi} \wedge k_{0}\left(x^{*}\right)+\gamma_{n}\left(x^{*}, e^{*}\right) k_{n}\left(x^{*}\right)=\bar{\pi}^{*}\right] \\
\leq \frac{1}{\widetilde{p}} \cdot \sum_{\left(k_{0}, k_{n}\right) \in K^{\prime}} \chi\left[k_{0}\left(x^{*}\right)+\gamma_{n}\left(x^{*}, e^{*}\right) k_{n}\left(x^{*}\right)=\bar{\pi}^{*}\right],
\end{array}
$$


where $K^{\prime}$ denotes the set of all $\left(k_{0}, k_{n}\right) \in \mathcal{H}^{2}$ satisfying that $k_{i}\left(g_{j}\right)=s_{i, j}$ for any $i \in\{0, n\}$ and $j \in\{1, \ldots, d\}$.

The inequality above becomes trivial if $K^{\prime}=\emptyset$; from now, we suppose that $K^{\prime} \neq \emptyset$. We take an element $\left(k_{0}^{*}, k_{n}^{*}\right)$ of $K^{\prime}$. Let $A$ denote the subgroup of $\mathcal{H}$ consisting of homomorphisms $\psi: X \rightarrow \Pi$ satisfying that $\psi(a)=0$ for all $a \in L$. Then any element of $K^{\prime}$ is uniquely expressed as $\left(k_{0}^{*}+\psi_{0}, k_{n}^{*}+\psi_{n}\right)$ with $\psi_{0}, \psi_{n} \in A$. Moreover, we take an element $\psi_{*}$ of $A$ satisfying that $\psi_{*}(x) \neq 0$, which exists since the group system $\mathbf{G}$ is diverse and $x \notin L$. Let $\operatorname{ord}\left(\psi_{*}\right)$ denote the order of the group element $\psi_{*} \in A$. Then there exist elements $\psi_{1}, \ldots, \psi_{\ell} \in A$ with $\ell=|A| / \operatorname{ord}\left(\psi_{*}\right)$ (namely, the representative elements of the cosets in the quotient group of $A$ by the subgroup generated by $\psi_{*}$ ) satisfying that any element of $A$ is uniquely expressed as $\psi_{i}+a \psi_{*}$ with $i \in\{1, \ldots, \ell\}$ and $a \in\left\{0,1, \ldots, \operatorname{ord}\left(\psi_{*}\right)-1\right\}$. Now if $k_{0}=k_{0}^{*}+\psi_{i_{0}}+a_{0} \psi_{*}$ and $k_{n}=k_{n}^{*}+\psi_{i_{n}}+a_{n} \psi_{*}$, then we have

$$
\begin{aligned}
k_{0}(x)+\gamma_{n}(x, e) k_{n}(x) & =k_{0}^{*}(x)+\psi_{i_{0}}(x)+\gamma_{n}(x, e)\left(k_{n}^{*}(x)+\psi_{i_{n}}(x)\right)+\left(a_{0}+\gamma_{n}(x, e) a_{n}\right) \psi_{*}(x), \\
k_{0}\left(x^{*}\right)+\gamma_{n}\left(x^{*}, e^{*}\right) k_{n}\left(x^{*}\right) & =k_{0}^{*}\left(x^{*}\right)+\psi_{i_{0}}\left(x^{*}\right)+\gamma_{n}\left(x^{*}, e^{*}\right)\left(k_{n}^{*}\left(x^{*}\right)+\psi_{i_{n}}\left(x^{*}\right)\right)+\left(a_{0}+\gamma_{n}(x, e) a_{n}\right) \psi_{*}\left(x^{*}\right) .
\end{aligned}
$$

Therefore, it suffices to show that, for any $k_{1}, \ldots, k_{n-1} \in \mathcal{H}$ and any $i_{0}, i_{n} \in\{1, \ldots, \ell\}$, we have

$$
\begin{array}{r}
\sum_{a_{0}, a_{n}=0}^{\operatorname{ord}\left(\psi_{*}\right)-1} \chi\left[\left(a_{0}+\gamma_{n}(x, e) a_{n}\right) \psi_{*}(x)=\pi^{\prime} \wedge\left(a_{0}+\gamma_{n}\left(x^{*}, e^{*}\right) a_{n}\right) \psi_{*}\left(x^{*}\right)=\pi^{\prime *}\right] \\
\leq \frac{1}{\widetilde{p}} \cdot \sum_{a_{0}, a_{n}=0}^{\operatorname{ord}\left(\psi_{*}\right)-1} \chi\left[\left(a_{0}+\gamma_{n}\left(x^{*}, e^{*}\right) a_{n}\right) \psi_{*}\left(x^{*}\right)=\pi^{\prime *}\right]
\end{array}
$$

where we put

$$
\begin{aligned}
\pi^{\prime} & =\bar{\pi}-k_{0}^{*}(x)-\psi_{i_{0}}(x)-\gamma_{n}(x, e)\left(k_{n}^{*}(x)+\psi_{i_{n}}(x)\right), \\
\pi^{\prime *} & =\bar{\pi}^{*}-k_{0}^{*}\left(x^{*}\right)-\psi_{i_{0}}\left(x^{*}\right)-\gamma_{n}\left(x^{*}, e^{*}\right)\left(k_{n}^{*}\left(x^{*}\right)+\psi_{i_{n}}\left(x^{*}\right)\right) .
\end{aligned}
$$

We show that $\psi_{*}(a \cdot x) \neq 0$ for any integer $a \neq 0$ with $|a|<\widetilde{p}$. First, $a$ is coprime to $|X / L|$ by the definition of $\widetilde{p}$, therefore we have $b_{1} a=b_{2}|X / L|+1$ for some integers $b_{1}, b_{2}$. Now we have $\psi_{*}\left(b_{2}|X / L| \cdot x\right)=0$ since $|X / L| \cdot x \in L$ (note that the order of the image of $x$ in the quotient group $X / L$ is a divisor of $|X / L|$ ), while $\psi_{*}(x) \neq 0$ by the choice of $\psi_{*}$. This implies that $\psi_{*}\left(b_{1} a \cdot x\right) \neq 0$, therefore $\psi_{*}(a \cdot x) \neq 0$, as desired.

The previous paragraph implies that $\operatorname{ord}\left(\psi_{*}\right) \geq \widetilde{p}$, since $\psi_{*}\left(\operatorname{ord}\left(\psi_{*}\right) \cdot x\right)=\left(\operatorname{ord}\left(\psi_{*}\right) \cdot \psi_{*}\right)(x)=0$. Now, since $\gamma_{n}(x, e), \gamma_{n}\left(x^{*}, e^{*}\right) \in\{0,1, \ldots, \widetilde{p}-1\}$ and $\gamma_{n}(x, e) \neq \gamma_{n}\left(x^{*}, e^{*}\right)$, the matrix $\left(\begin{array}{cc}1 & \gamma_{n}(x, e) \\ 1 & \gamma_{n}\left(x^{*}, e^{*}\right)\end{array}\right)$ is nonsingular, where the components are considered modulo ord $\left(\psi_{*}\right)$. This implies that, when $a_{0}$ and $a_{n}$ run over $\left\{0,1, \ldots, \operatorname{ord}\left(\psi_{*}\right)-1\right\}$, the pair of $\left(a_{0}+\gamma_{n}(x, e) a_{n} \bmod \operatorname{ord}\left(\psi_{*}\right)\right)$ and $\left(a_{0}+\gamma_{n}\left(x^{*}, e^{*}\right) a_{n} \bmod \operatorname{ord}\left(\psi_{*}\right)\right)$ distributes uniformly on $\left\{0,1, \ldots, \operatorname{ord}\left(\psi_{*}\right)-1\right\}^{2}$. Therefore, it suffices to show that, for any $k_{1}, \ldots, k_{n-1} \in \mathcal{H}$ and any $i_{0}, i_{n} \in\{1, \ldots, \ell\}$, we have

$$
\sum_{a, a^{\prime}=0}^{\operatorname{ord}\left(\psi_{*}\right)-1} \chi\left[a \psi_{*}(x)=\pi^{\prime} \wedge a^{\prime} \psi_{*}\left(x^{*}\right)=\pi^{\prime *}\right] \leq \frac{1}{\widetilde{p}} \cdot \sum_{a, a^{\prime}=0}^{\operatorname{ord}\left(\psi_{*}\right)-1} \chi\left[a^{\prime} \psi_{*}\left(x^{*}\right)=\pi^{\prime *}\right] .
$$

Now we note that, the condition $a \psi_{*}(x)=\pi^{\prime}$ is satisfied by at most $\operatorname{ord}\left(\psi_{*}\right) / \widetilde{p}$ integers $a \in\left\{0,1, \ldots, \operatorname{ord}\left(\psi_{*}\right)-\right.$ $1\}$. Indeed, if the number of such $a$ is larger than $\operatorname{ord}\left(\psi_{*}\right) / \widetilde{p}$, then the pigeonhole principle implies that we have $a_{1} \psi_{*}(x)=a_{2} \psi_{*}(x)=\pi^{\prime}$ for some integers $a_{1}<a_{2}$ with $a_{2}-a_{1}<\widetilde{p}$. However, this implies that $\psi_{*}\left(\left(a_{2}-a_{1}\right) \cdot x\right)=\left(a_{2}-a_{1}\right) \psi_{*}(x)=0$, contradicting the previous paragraph. Hence, we have

$$
\begin{aligned}
\sum_{a, a^{\prime}=0}^{\operatorname{ord}\left(\psi_{*}\right)-1} \chi\left[a \psi_{*}(x)=\pi^{\prime} \wedge a^{\prime} \psi_{*}\left(x^{*}\right)=\pi^{\prime *}\right] & \leq \sum_{a^{\prime}=0}^{\operatorname{ord}\left(\psi_{*}\right)-1} \frac{\operatorname{ord}\left(\psi_{*}\right)}{\widetilde{p}} \chi\left[a^{\prime} \psi_{*}\left(x^{*}\right)=\pi^{\prime *}\right] \\
& =\frac{1}{\widetilde{p}} \cdot \sum_{a, a^{\prime}=0}^{\operatorname{ord}\left(\psi_{*}\right)-1} \chi\left[a^{\prime} \psi_{*}\left(x^{*}\right)=\pi^{\prime *}\right]
\end{aligned}
$$

as desired. This completes the proof of Lemma 5.1. 
Owing to Lemma 5.1, we will show that the CS projective hash family is (information-theoretically or computationally) universal 2 , if the internal function $\Gamma$ satisfies some appropriate property. First, we recall the notions of collision resistant (CR) hash family and target collision resistant (TCR) hash family.

Definition 5.1 (Collision Resistant Hash Family). Let $\left\{f_{h k} \mid h k \in \mathcal{H K}\right\}$ be a family of hash functions $f_{h k}: \mathcal{X} \rightarrow \mathcal{Y}$ indexed by a hash key $h k \in \mathcal{H} \mathcal{K}$. We say that the family is collision resistant (CR), if for any PPT adversary $\mathcal{A}$, its advantage $A d v_{\mathcal{A}}^{\mathrm{CR}}(\ell)$ defined by

$$
A d v_{\mathcal{A}}^{\mathrm{CR}}(\ell)=\operatorname{Pr}\left[h k \stackrel{\$}{\leftarrow} \mathcal{H} \mathcal{K} ;\left(x, x^{*}\right) \leftarrow \mathcal{A}\left(1^{\ell}, h k\right): x \neq x^{*} \wedge f_{h k}(x)=f_{h k}\left(x^{*}\right)\right]
$$

is negligible in the security parameter $\ell$.

Definition 5.2 (Target Collision Resistant Hash Family). Let $\left\{f_{h k} \mid h k \in \mathcal{H} \mathcal{K}\right\}$ be a family of hash functions $f_{h k}: \mathcal{X} \rightarrow \mathcal{Y}$ indexed by a hash key $h k \in \mathcal{H} \mathcal{K}$. Let $\mathcal{X}^{\prime} \subset \mathcal{X}$. We say that the family is target collision resistant (TCR) relative to $\mathcal{X}^{\prime}$, if for any PPT adversary $\mathcal{A}$, its advantage $\operatorname{Adv}_{\mathcal{A}}^{\mathrm{TCR}}(\ell)$ defined by

$$
A d v_{\mathcal{A}}^{\mathrm{TCR}}(\ell)=\operatorname{Pr}\left[x^{*} \stackrel{\$}{\leftarrow} \mathcal{X}^{\prime} ; h k \stackrel{\$}{\leftarrow} \mathcal{H} \mathcal{K} ; x \leftarrow \mathcal{A}\left(1^{\ell}, h k, x^{*}\right): x \neq x^{*} \wedge f_{h k}(x)=f_{h k}\left(x^{*}\right)\right]
$$

is negligible in the security parameter $\ell$. When $\mathcal{X}^{\prime}=\mathcal{X}$, we simply say that the family of hash functions is target collision resistant.

On the other hand, we also introduce a simple but useful technique to improve the efficiency; we can "compress" the output of the CS projective hash family by using a "smooth" function. The smoothness of a function defined below is a statistical property that roughly ensures that the "min-entropy" of the output of the function (for uniformly random input) is sufficiently high, and thus it is information-theoretically hard to guess the output. ${ }^{5}$ The definition is as follows.

Definition 5.3 (Smooth Function). Let $f: \mathcal{X} \rightarrow \mathcal{Y}$ be a hash function. We say that $f$ is $\epsilon$-smooth, if the quantity $\operatorname{Smth}_{f}:=\max _{y \in \mathcal{Y}} \underset{\substack{\mathscr{\&} \\ \text { Pr }}}{\operatorname{Pr}}[f(x)=y]$ is not larger than $\epsilon$. We say that $f$ is smooth, if it is $\epsilon$-smooth for a negligible $\epsilon$.

We note that, besides the injective functions (with superpolynomially large domain) which are trivially smooth, the smoothness is in fact satisfied by several famous cryptographic functions such as OWFs, always second-preimage resistant (aSec secure) hash functions [41], and KDFs [16]; see Appendix A. Interestingly, the universal ${ }_{2}$ property of the CS projective hash family is preserved by hashing its output to be a shorter element. Intuitively, for the CS projective hash family, the bound of the advantage of adversaries for the universal $_{2}$ property is closely related to the parameter for the underlying subset membership problem $\mathbf{M}$, therefore the bound cannot be freely selected (e.g., the order of the group should be larger than a certain threshold relevant to the desired security level) since $\mathbf{M}$ must be hard. Our proposed technique provides a way to reduce the output size of the projective hash family, while the too strong bound of the universal 2 advantage is increased but is still reasonably strong. It is a bit surprising that this technique can be also applied to the original Cramer-Shoup scheme, but to the best of our knowledge, it has never explicitly been stated in the literature. When applying our technique to the Cramer-Shoup scheme, ciphertext length of the resulting scheme becomes the same as that of the Kurosawa-Desmedt (KD) scheme [35] which is the best known DDH-based PKE scheme. We should also note that this technique is not applicable to other similar schemes such as the Cash-Kiltz-Shoup [11], Hanaoka-Kurosawa [26], and Kiltz schemes [33]. This fact is primarily due to the structure of HPS-based constructions, and thus, it is difficult to apply the above technique to PKE schemes from other methodology, e.g. [8, 26, 32].

We describe the technique discussed above. For any projective hash family $\mathbf{H}$ and any smooth function $f$ with domain including $\Pi$, we define the composition $f \circ \mathbf{H}$ to be the projective hash family obtained from $\mathbf{H}$ by taking the composition $f \circ H_{k}$ for the function $H_{k}, k \in K$. We will show that, for the case that $\mathbf{H}$ is the CS projective hash family, $f \circ \mathbf{H}$ is (information-theoretically or computationally) universal ${ }_{2}$ provided some appropriate conditions are satisfied. For the purpose, we require a trapdoor property for the underlying subset membership problem, formalized as follows.

\footnotetext{
${ }^{5}$ Note that this notion is (somewhat similar to but) different from the smoothness of a projective hash family.
} 
Definition 5.4 (Trapdoor Subset Membership Problem). We say that a subset membership problem $\mathbf{M}$ is a trapdoor subset membership problem, if it is endowed with an additional trapdoor mode as well as the ordinary mode, satisfying the followings: (1) In the trapdoor mode, the instance sampling algorithm takes as input $1^{\ell}$ and returns $\Lambda=\Lambda\left[X, X^{\prime}, L, W, R\right] \in\left[I_{\ell}\right]$ and a trapdoor element $\tau$, where the distribution of $\Lambda$ in the trapdoor mode is identical to that of $\Lambda$ in the ordinary mode; (2) there exists a PPT algorithm that takes the trapdoor $\tau$ and an element $x \in X$ as input and decides whether $x \in L$ or not.

We say that a trapdoor subset membership problem $\mathbf{M}$ is hard (relative to $X^{\prime}$ ), if it is hard (relative to $\left.X^{\prime}\right)$ in the ordinary mode as a subset membership problem.

Based on the definitions above, we give the following result:

Proposition 5.1. Let $\mathbf{H}$ be the CS projective hash family constructed as above. Let $f: \Pi \rightarrow \mathcal{Y}$ be an $\epsilon$-smooth hash function.

1. If the function $\Gamma$ is injective, then $f \circ \mathbf{H}$ is $(|\Pi| \epsilon / \widetilde{p})$-universal ${ }_{2}$.

2. If $\Gamma$ is sampled from a CR hash family, the subset membership problem associated to $\mathbf{H}$ is a trapdoor subset membership problem, and $|\Pi| \epsilon / \widetilde{p}$ is negligible, then $f \circ \mathbf{H}$ is first-adaptive computationally universal 2 .

3. If $\Gamma$ is sampled from a TCR hash family relative to a subset $X^{\prime} \times E \subset X \times E$, the subset membership problem associated to $\mathbf{H}$ is a trapdoor subset membership problem, and $|\Pi| \epsilon / \widetilde{p}$ and $\left|X^{\prime} \cap L\right| /\left|X^{\prime}\right|$ are negligible, then $f \circ \mathbf{H}$ is first-uniform computationally universal ${ }_{2}$ relative to $X^{\prime} \times E$.

Proof. For the first part, let $(x, e),\left(x^{*}, e^{*}\right) \in(X \backslash L) \times E$ with $(x, e) \neq\left(x^{*}, e^{*}\right)$, let $\vec{s} \in S$, and let $y, y^{*} \in \mathcal{Y}$. Then we have

$$
\begin{aligned}
& \operatorname{Pr}_{\vec{k}}\left[f \circ H_{\vec{k}}(x, e)=y \wedge f \circ H_{\vec{k}}\left(x^{*}, e^{*}\right)=y^{*} \wedge \alpha(\vec{k})=\vec{s}\right] \\
& =\sum_{\pi \in f^{-1}(y), \pi^{*} \in f^{-1}\left(y^{*}\right)} \operatorname{Pr}_{\vec{k} \stackrel{\$}{\leftarrow}}\left[H_{\vec{k}}(x, e)=\pi \wedge H_{\vec{k}}\left(x^{*}, e^{*}\right)=\pi^{*} \wedge \alpha(\vec{k})=\vec{s}\right] .
\end{aligned}
$$

Since $\Gamma$ is injective, we have $\Gamma(x, e) \neq \Gamma\left(x^{*}, e^{*}\right)$, therefore Lemma 5.1 implies that the right-hand side of the last equality is not larger than

$$
\begin{aligned}
& \sum_{\pi \in f^{-1}(y), \pi^{*} \in f^{-1}\left(y^{*}\right)} \frac{1}{\widetilde{p}} \cdot \operatorname{Pr}_{\vec{k} \stackrel{\mathbb{E}}{\leftarrow} K}\left[H_{\vec{k}}\left(x^{*}, e^{*}\right)=\pi^{*} \wedge \alpha(\vec{k})=\vec{s}\right] \\
& =\frac{\left|f^{-1}(y)\right|}{\widetilde{p}} \cdot \operatorname{Pr}_{\vec{k} \stackrel{\$}{\leftarrow}\left[f \circ H_{\vec{k}}\left(x^{*}, e^{*}\right)=y^{*} \wedge \alpha(\vec{k})=\vec{s}\right] .}
\end{aligned}
$$

This implies that $f \circ \mathbf{H}$ is $\left(\max _{y \in \mathcal{Y}}\left|f^{-1}(y)\right| / \widetilde{p}\right)$-universal 2 . Moreover, we have $\operatorname{Smth}_{f}=\max _{y \in \mathcal{Y}}\left|f^{-1}(y)\right| /|\Pi|$, therefore $\max _{y \in \mathcal{Y}}\left|f^{-1}(y)\right| \leq|\Pi| \epsilon$ since $f$ is $\epsilon$-smooth. Hence $f \circ \mathbf{H}$ is $(|\Pi| \epsilon / \widetilde{p})$-universal ${ }_{2}$, as desired.

For the second part of the claim, let $\mathcal{A}$ be an adversary for the first-adaptive computationally universal $_{2}$ game of $f \circ \mathbf{H}$. Let $\tau$ denote the trapdoor element for the subset membership problem associated to $\mathbf{H}$ generated in its trapdoor mode. Then we construct an adversary $\mathcal{A}^{\dagger}$ for the $\mathrm{CR}$ property for $\Gamma$ in the following manner. The adversary $\mathcal{A}^{\dagger}$ first generates the key $\vec{k} \in K$ uniformly at random, computes $\vec{s}=\alpha(\vec{k})$, and then executes the adversary $\mathcal{A}$ with input $\vec{s}$. In the simulation of the first-adaptive computationally universal $_{2}$ game, $\mathcal{A}^{\dagger}$ emulates the oracle Hash by using the trapdoor element $\tau$ (to efficiently decide whether the query $(x, e)$ is in $L \times E$ or not) and the key $\vec{k}$ (to compute the reply $H_{\vec{k}}(x, e)$ to the query). Similarly, given an element $\left(x^{*}, e^{*}\right) \in X \times E$ submitted by $\mathcal{A}, \mathcal{A}^{\dagger}$ computes the reply $y^{*}=f \circ H_{\vec{k}}\left(x^{*}, e^{*}\right)$ by using $\vec{k}$. Finally, $\mathcal{A}^{\dagger}$ receives an output $((x, e), y) \in(X \times E) \times \mathcal{Y}$ of $\mathcal{A}$, and outputs $(x, e)$ and $\left(x^{*}, e^{*}\right)$. Now let $T$ (respectively, $\left.T^{\prime}\right)$ denote the event that $\Gamma(x, e)=\Gamma\left(x^{*}, e^{*}\right)\left(\right.$ respectively, $\left.\Gamma(x, e) \neq \Gamma\left(x^{*}, e^{*}\right)\right)$ and $\mathcal{A}$ wins the first-adaptive computationally universal 2 game. Then we have $A d v_{\mathcal{A}}^{\text {AComp.Univ }_{2}}(\ell)=\operatorname{Pr}[T]+\operatorname{Pr}\left[T^{\prime}\right]$ and 
$\operatorname{Pr}[T] \leq A d v_{\mathcal{A}^{\dagger}}^{\mathrm{CR}}(\ell)$. Moreover, the same argument as the previous paragraph based on Lemma 5.1 implies that

$$
\operatorname{Pr}\left[T^{\prime}\right]=\operatorname{Pr}\left[\Gamma(x, e) \neq \Gamma\left(x^{*}, e^{*}\right) \wedge H_{\vec{k}}(x, e)=y \mid H_{\vec{k}}\left(x^{*}, e^{*}\right)=y^{*} \wedge \alpha(\vec{k})=\vec{s}\right] \leq \frac{|\Pi| \epsilon}{\widetilde{p}},
$$

which is negligible by the assumption. Hence, $A d v_{\mathcal{A}^{\dagger}}^{\mathrm{CR}}(\ell)$ is non-negligible provided $A d v_{\mathcal{A}}^{\mathrm{AComp}^{\text {Univ }}}{ }_{2}(\ell)$ is nonnegligible. This completes the proof of the second part of the claim.

Similarly, for the third part of the claim, let $\mathcal{A}$ be an adversary for the first-uniform computationally universal $_{2}$ game of $f \circ \mathbf{H}$ relative to $X^{\prime} \times E$. Let $\tau$ denote the trapdoor element for the subset membership problem associated to $\mathbf{H}$ generated in its trapdoor mode. Then we construct an adversary $\mathcal{A}^{\dagger}$ for the TCR property for $\Gamma$ relative to $X^{\prime} \times E$ in the following manner. Given an input $\left(x^{*}, e^{*}\right) \in X^{\prime} \times E$, the adversary $\mathcal{A}^{\dagger}$ first generates the key $\vec{k} \in K$ uniformly at random, computes $\vec{s}=\alpha(\vec{k})$, and then executes the adversary $\mathcal{A}$ with input $\left(x^{*}, e^{*}\right), \vec{s}$ and $y^{*}=f \circ H_{\vec{k}}\left(x^{*}, e^{*}\right)$. Here $\mathcal{A}^{\dagger}$ efficiently simulates the first-uniform computationally universal 2 game by using $\tau$ and $\vec{k}$ in the same manner as the previous paragraph. Finally, $\mathcal{A}^{\dagger}$ receives an output $((x, e), y) \in(X \times E) \times \mathcal{Y}$ of $\mathcal{A}$, and outputs $(x, e)$ and $\left(x^{*}, e^{*}\right)$. We define the events $T$ and $T^{\prime}$ in the same manner as in the previous paragraph. Moreover, let $T_{0}$ and $T_{0}^{\prime}$ denote the same events as $T$ and $T^{\prime}$, respectively, except that the input $\left(x^{*}, e^{*}\right)$ for $\mathcal{A}^{\dagger}$ is chosen uniformly at random from $\left(X^{\prime} \backslash L\right) \times E$ instead of $X^{\prime} \times E$. Then we have $A d v_{\mathcal{A}}^{\text {UComp.Univ }_{2}}(\ell)=\operatorname{Pr}\left[T_{0}\right]+\operatorname{Pr}\left[T_{0}^{\prime}\right]$. On the other hand, by the assumption that $\left|X^{\prime} \cap L\right| /\left|X^{\prime}\right|$ is negligible, it follows that the uniform distributions on $X^{\prime} \times E$ and on $\left(X^{\prime} \backslash L\right) \times E$ have negligible statistical distance, therefore $\left|\operatorname{Pr}[T]-\operatorname{Pr}\left[T_{0}\right]\right|$ is negligible. Moreover, we have $\operatorname{Pr}[T] \leq A d v_{\mathcal{A}^{\dagger}}^{\mathrm{TCR}}(\ell)$, while the same argument as the previous paragraph implies that $\operatorname{Pr}\left[T_{0}^{\prime}\right] \leq|\Pi| \epsilon / \widetilde{p}$, which is negligible by the

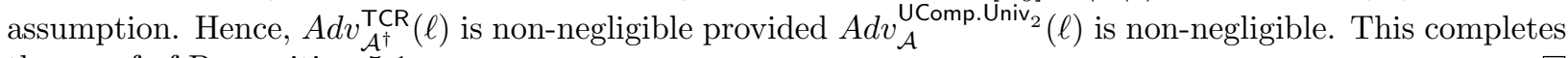
the proof of Proposition 5.1.

\subsection{Instantiation of KH-PKE from Diverse Group Systems}

Here we give an instantiation of our generic construction of KH-PKE schemes proposed in Section 4, based on a general diverse group system and the corresponding CS projective hash family. Let $\mathbf{G}=(\mathcal{H}, X, L, \Pi)$ be a diverse group system for which the associated subset membership problem is hard. Let $\widetilde{p}$ denote the smallest prime dividing $|X / L|$. Suppose that $\epsilon^{\prime}:=1 / \widetilde{p}$ and $\epsilon:=\left(\epsilon^{\prime}|\Pi|-1\right)(|\Pi|-1) / 2$ are both negligible. In the setting, we define the three hash proof systems $\mathbf{P}, \widehat{\mathbf{P}}$ and $\widetilde{\mathbf{P}}$ used by our generic construction in the following manner:

- We set $\mathbf{H}$ to be the homomorphic projective hash family constructed from $\mathbf{G}$ as mentioned in Section 2, and set $\mathbf{P}$ to be the corresponding hash proof system. Then $\mathbf{P}$ is $\epsilon$-smooth by Lemma 2.3 and Lemma 2.2 , and $\epsilon$ is negligible as above.

- We set $\widehat{\mathbf{H}}=\mathbf{H}$ and $\widehat{\mathbf{P}}=\mathbf{P}$. Then $\widehat{\mathbf{P}}$ is homomorphic and $\epsilon^{\prime}$-universal ${ }_{1}$ by Lemma 2.3 , and $\epsilon^{\prime}$ is negligible as above.

- Let $f: \Pi \rightarrow \mathcal{Y}$ be an $\epsilon_{\text {smth }}$-smooth function, and suppose that $\epsilon^{\prime \prime}:=|\Pi| \epsilon_{\text {smth }} / \widetilde{p}$ is negligible. We put $E:=\Pi^{2}$, and let $\Gamma: X \times E \rightarrow\{0,1, \ldots, \widetilde{p}-1\}^{n}$ be any injective function. We set $\widetilde{\mathbf{H}}$ to be the composition of $f$ and the CS projective hash family constructed from the diverse group system $\mathbf{G}$ and the internal function $\Gamma$, and set $\widetilde{\mathbf{P}}$ to be the corresponding hash proof system. Then $\widetilde{\mathbf{P}}$ is (information-theoretically) $\epsilon^{\prime \prime}$-universal 2 by Lemma 2.3 and Proposition 5.1, and $\epsilon^{\prime \prime}$ is negligible as above.

Then the conditions of Theorem 4.1 with Assumption I are satisfied, therefore the resulting instantiation of our KH-PKE scheme is KH-CCA secure.

For example, we can use any $\mathbf{G}$ satisfying that $|\Pi|=|X / L|$ and it is an exponentially large prime $\widetilde{p}$, and the identity map $\Pi \rightarrow \Pi$ as the smooth function $f$. Then we have $\epsilon=0, \epsilon^{\prime}=1 / \widetilde{p}, \epsilon_{\text {smth }}=1 /|\Pi|$ and $\epsilon^{\prime \prime}=1 / \widetilde{p}$, therefore all of $\epsilon, \epsilon^{\prime}$ and $\epsilon^{\prime \prime}$ are negligible, as desired. 


\subsection{DDH-Based Instantiation of KH-PKE}

From now, we give instantiations of our KH-PKE schemes based on some standard computational assumptions. First, we describe the instantiation based on the DDH assumption. We recall the definition of the DDH assumption.

Definition 5.5 (The Decisional Diffie-Hellman (DDH) Assumption). Let $\mathbb{G}$ be a multiplicative cyclic group of prime order $p$. We say that the $\mathrm{DDH}$ assumption holds in $\mathbb{G}$, if for any PPT algorithm $\mathcal{A}$, the advantage $A d v_{\mathbb{G}, \mathcal{A}}^{D D H}(\ell):=\left|\operatorname{Pr}\left[\mathcal{A}\left(g_{0}, g_{1}, g_{0}^{r}, g_{1}^{r}\right)=0\right]-\operatorname{Pr}\left[\mathcal{A}\left(g_{0}, g_{1}, g_{0}^{r}, g_{1}^{r^{\prime}}\right)=0\right]\right|$ is negligible, where $g_{0}$ and $g_{1}$ are chosen from $\mathbb{G}$ uniformly at random, and $r$ and $r^{\prime}$ are chosen from $\mathbb{Z}_{p}$ uniformly at random.

In order to construct the DDH-based instantiation, we define a trapdoor subset membership problem $\mathbf{M}$ and a diverse group system $\mathbf{G}$ in the following manner. Let $\mathbb{G}$ be a cyclic group of prime order $p$ for which the DDH assumption holds. In particular, $1 / p$ is negligible (since otherwise the DDH assumption is not satisfied), therefore the uniform distributions on $\mathbb{G}$ and on $\mathbb{G} \backslash\{1\}$ have negligible statistical distance.

- The instance sampling algorithm for $\mathbf{M}$ chooses two generators $g_{0}, g_{1} \in \mathbb{G} \backslash\{1\}$ of $\mathbb{G}$ uniformly at random, sets $X:=\mathbb{G}^{2}, L:=\left\{\left(g_{0}^{i}, g_{1}^{i}\right) \in X \mid i \in \mathbb{Z}_{p}\right\} \simeq \mathbb{G}$ which is generated by $\left(g_{0}, g_{1}\right), W:=\mathbb{Z}_{p}$, and defines the relation $R$ in such a way that, for $\left(x_{0}, x_{1}\right) \in X$ and $\omega \in W$, we have $\left(\left(x_{0}, x_{1}\right), \omega\right) \in R$ if and only if $x_{0}=g_{0}^{\omega}$ and $x_{1}=g_{1}^{\omega}$. On the other hand, the subset sampling algorithm first chooses $\omega \in W$ uniformly at random, and then outputs $\left(g_{0}^{\omega}, g_{1}^{\omega}\right) \in L$ and the $\omega \in W$. The construction of $\mathbf{M}$ satisfies the condition for a hard subset membership problem, where the hardness follows immediately from the DDH assumption on $\mathbb{G}$.

- In the trapdoor mode for $\mathbf{M}$, the algorithm chooses the $g_{0}$ and $g_{1}$ above in such a way that $g_{0}$ is chosen first; secondly $\tau \in \mathbb{Z}_{p} \backslash\{0\}$ is chosen uniformly at random, which is the trapdoor element; and then $g_{1}$ is defined by $g_{1}:=g_{0}^{\tau}$. Then, by using $\tau$, it can be efficiently decided whether a given $\left(x_{0}, x_{1}\right) \in X$ is in $L$ or not, by checking if $x_{1}=x_{0}^{\tau}$. Hence, $\mathbf{M}$ is a hard trapdoor subset membership problem.

- To define the corresponding diverse group system $\mathbf{G}$, we set $\Pi:=\mathbb{G}$, and define $\mathcal{H}$ to be the set of homomorphisms $H_{k_{0}, k_{1}}: X \rightarrow \Pi$, indexed by $\left(k_{0}, k_{1}\right) \in \mathbb{Z}_{p}^{2}$, satisfying that $H_{k_{0}, k_{1}}\left(x_{0}, x_{1}\right):=x_{0}^{k_{0}} x_{1}^{k_{1}}$ for any $\left(x_{0}, x_{1}\right) \in X$. Then $\mathbf{G}$ is diverse; indeed, for any $\left(x_{0}, x_{1}\right)=\left(g_{0}^{i}, g_{1}^{j}\right) \in X$, by putting $g_{1}=g_{0}^{\tau}$, we have $H_{-\tau, 1}\left(x_{0}, x_{1}\right)=g_{0}^{(j-i) \tau}=1$ if and only if $j \equiv i \bmod p$, i.e., $\left(x_{0}, x_{1}\right) \in L$.

By the construction, we have $|X / L|=|\Pi|=p$, therefore the homomorphic hash proof system $\mathbf{P}=\widehat{\mathbf{P}}$ associated to the $\mathbf{M}$ and $\mathbf{G}$ is $(1 / p)$ - universal $_{1}$ (by Lemma 2.3) and 0-smooth (by Lemma 2.2). On the other hand, let $\Gamma=\Gamma_{h k}: X \times \Pi^{2} \rightarrow\{0,1, \ldots, p-1\}$ be a function indexed by $h k \in \mathcal{H} \mathcal{K}$ sampled from a TCR hash family. Let $f: \Pi \rightarrow \mathcal{Y}$ be an $\epsilon_{\text {smth }}$-smooth function, where $\epsilon_{\text {smth }}$ is negligible. Then, since $\mathbf{M}$ is a trapdoor subset membership problem and the values $|\Pi| \epsilon_{\mathrm{smth}} / p=\epsilon_{\mathrm{smth}}$ and $|L| /|X|=1 / p$ are negligible, Proposition 5.1 implies that the composition (denoted by $\widetilde{\mathbf{P}}$ ) of $f$ and the CS hash proof system constructed from the diverse group system $\mathbf{G}$ and the internal function $\Gamma$ is a first-uniform computationally universal ${ }_{2}$ hash proof system.

Now we show that the conditions of Theorem 4.1 with Assumption $U$ (where $X^{\prime}=X$ and $\Pi^{\prime}=\Pi$ ) are satisfied (note that the last two conditions in Assumption $\mathrm{U}$ are now trivial, since $X^{\prime}=X$ ). First, since $|L| /|X|=1 / p$ is negligible, the uniform distributions on $X$ and on $X \backslash L$ have negligible statistical distance, therefore $X \backslash L$ is approximately samplable relative to $X$. Secondly, for the condition for critical integers, since $|X / L|=p$, we have $o(\Lambda)=p$. On the other hand, we have $|X|=p^{2}$, therefore any positive integer that is not coprime to $|X|$ is a multiple of $p$. This implies that there exist no critical integers, therefore the condition for critical integers in Assumption $U$ is automatically satisfied. Hence, all the conditions for Theorem 4.1 with Assumption U are satisfied, therefore the resulting instantiation of our KH-PKE scheme is KH-CCA secure. We write down the instantiation of the KH-PKE scheme in Figure 2. 


\begin{tabular}{|c|c|}
\hline 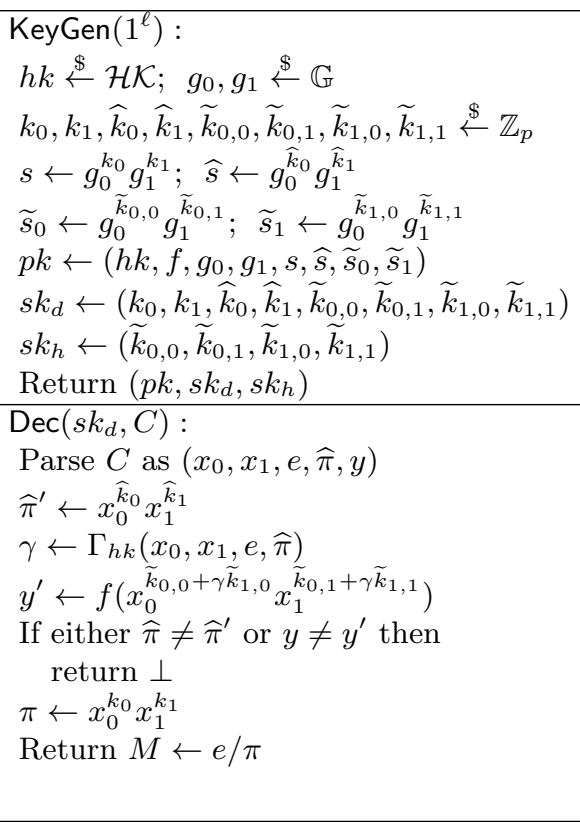 & 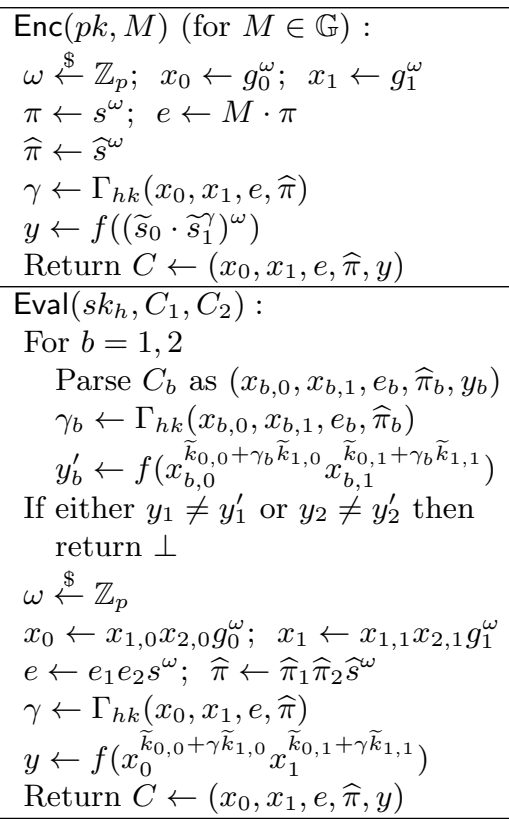 \\
\hline
\end{tabular}

Figure 2: DDH-based instantiation of our KH-PKE scheme. Here $\mathbb{G}$ is a cyclic group of prime order $p$ satisfying the DDH assumption; $\left\{\Gamma=\Gamma_{h k}: \mathbb{G}^{4} \rightarrow\{0,1, \ldots, p-1\} \mid h k \in \mathcal{H} \mathcal{K}\right\}$ is a TCR hash family; and $f: \mathbb{G} \rightarrow \mathcal{Y}$ is a smooth function.

Efficiency Comparison: In Table 7, we give an efficiency comparison of our DDH-based KH-PKE scheme with the CS PKE [13], the KD PKE [35], and the naive construction (see Section 1). We note that the latter three schemes do not possess the keyed-homomorphic property and/or the KH-CCA security. As seen in Table 7, our scheme is comparably efficient to the best known DDH-based (standard) PKE schemes, i.e. the $\mathrm{CS}$ and the KD schemes, in terms of computational costs. The ciphertext size of our construction is dependent on how large the output length $n$ of the smooth function $f$ is. However, as analyzed in Appendix A, if we assume that $f$ is a OWF, an aSec hash function, or a KDF, that is secure against non-uniform adversaries, then $n$ can be as small as $\ell$ for $\ell$-bit security. In this case, the ciphertext overhead of our scheme is only $\ell$-bit longer than that of the CS scheme for $\ell$-bit security. ${ }^{6}$ Then, for 128-bit security, ciphertext overhead of our scheme is 896-bit while that of the Cramer-Shoup scheme is 768-bit (assuming that these schemes are implemented over elliptic curves).

It is somewhat surprising that it is possible to realize KH property with only significantly small additional cost. Furthermore, comparing with the naive construction (from KD and CS(-lite)) which appears to have KH property (but does not satisfy KH-CCA security), we see that our scheme is more efficient. This means that our methodology does not only yield KH property (and KH-CCA security) but also significantly high efficiency.

\subsection{DCR-Based Instantiation of KH-PKE}

Here we describe the instantiation of our KH-PKE scheme based on the DCR assumption. First, we recall the definition of the DCR assumption.

Definition 5.6 (The Decisional Composite Residuosity (DCR) Assumption [40]). Let $p, q, p^{\prime}, q^{\prime}$ be distinct odd primes with $p=2 p^{\prime}+1$ and $q=2 q^{\prime}+1$, where $p^{\prime}$ and $q^{\prime}$ are both $\lambda=\lambda(\ell)$ bits in length. Let

\footnotetext{
${ }^{6}$ Even if this "non-uniform" security assumption is not justified (and only security against uniform PPT adversaries is assumed), $n$ can still be as small as at most $2 \ell$-bit, which is still smaller than (or in some group equal to) the size of an element in the group $\mathbb{G}$.See our analysis of smoothness of these cryptographic functions in Appendix A.
} 
Table 7: Comparison among the Cramer-Shoup (CS) scheme, the Kurosawa-Desmedt (KD) scheme, the KD + CSlite (using the double encryption) scheme, and our DDH-based KH-PKE scheme (here $|C|-|M|$ denotes ciphertext overhead; $|g|$ denotes the size of an element in the underlying group $\mathbb{G}$; exp denotes exponentiation; and we count 1 multi-exp as 1.2 regular exp, and the size of MAC and the output length of $f$ as $\ell$ and $n=n(\ell)$, respectively)

\begin{tabular}{|c||c|c|c|c|}
\hline & $|C|-|M|$ & Cost (Enc) & Cost (Dec) & KH property \\
\hline \hline CS [13] & $3|g|$ & $4.2 \exp$ & $2.4 \exp$ & No \\
\hline KD [35] & $2|g|+\ell$ & $3.2 \exp$ & $1.2 \exp$ & No \\
\hline KD+CS-lite Double Enc & $5|g|+\ell$ & $7.2 \exp$ & $3.6 \exp$ & No? \\
\hline Our DDH-based KH-PKE & $3|g|+n$ & $5.4 \exp$ & $3.6 \exp$ & Yes \\
\hline
\end{tabular}

$N=p q . \quad$ We say that the DCR assumption holds in $\mathbb{Z}_{N^{2}}^{*}$, if for any PPT adversary $\mathcal{A}$, the advantage $A d v_{N, \mathcal{A}}^{D C R}\left(1^{\ell}\right):=\left|\operatorname{Pr}[\mathcal{A}(g, N)=0]-\operatorname{Pr}\left[\mathcal{A}\left(g^{N}, N\right)=0\right]\right|$ is negligible, where $g$ is a uniformly random element of $\mathbb{Z}_{N^{2}}^{*}$.

In order to construct the DCR-based instantiation, we note the following immediate consequence of the DCR assumption:

Lemma 5.2. Let $p, q, p^{\prime}, q^{\prime}$ and $N=p q$ be as in the definition of the DCR assumption. If the DCR assumption holds in $\mathbb{Z}_{N^{2}}^{*}$, then $\left|\operatorname{Pr}\left[\mathcal{A}\left(g^{2}, N\right)=0\right]-\operatorname{Pr}\left[\mathcal{A}\left(g^{2 N}, N\right)=0\right]\right|$ is negligible for any PPT adversary $\mathcal{A}$, where $g$ is a uniformly random element of $\mathbb{Z}_{N^{2}}^{*}$.

We define a trapdoor subset membership problem $\mathbf{M}$ and a diverse group system $\mathbf{G}$ as follows.

- The instance sampling algorithm for $\mathbf{M}$ chooses the primes $p, q, p^{\prime}$ and $q^{\prime}$ as in the DCR assumption, puts $N:=p q$, and sets $X:=\left\{g^{2} \mid g \in \mathbb{Z}_{N^{2}}^{*}\right\}$ and $L:=\left\{g^{2 N} \mid g \in \mathbb{Z}_{N^{2}}^{*}\right\}$. By the choice of $N$, we have $\mathbb{Z}_{N^{2}}^{*} \simeq \mathbb{Z}_{p^{2}}^{*} \times \mathbb{Z}_{q^{2}}^{*} \simeq\left(C_{p} \times C_{2} \times C_{p^{\prime}}\right) \times\left(C_{q} \times C_{2} \times C_{q^{\prime}}\right)$ where $C_{n}$ denotes the multiplicative cyclic group of order $n$, therefore $X \simeq C_{p} \times C_{p^{\prime}} \times C_{q} \times C_{q^{\prime}}$ and $L \simeq C_{p^{\prime}} \times C_{q^{\prime}}$. Let $\iota$ denote the isomorphism from $X$ to $C_{p} \times C_{p^{\prime}} \times C_{q} \times C_{q^{\prime}}$. Moreover, $X^{\prime}$ is defined to be the subset of $X$ consisting of elements $\iota^{-1}(y)$ with $y=\left(y_{p}, y_{p^{\prime}}, y_{q}, y_{q^{\prime}}\right) \in C_{p} \times C_{p^{\prime}} \times C_{q} \times C_{q^{\prime}}$ satisfying either $y_{p} \neq 1$ and $y_{q} \neq 1$, or $y_{p}=y_{q}=1$. Now let $g_{*}$ be a generator of $L$, which can be approximately sampled by $g_{*}=g^{2 N}$ where $g \in \mathbb{Z}_{N^{2}}^{*}$ is chosen uniformly at random. ${ }^{7}$ Then the instance sampling algorithm sets $W:=\{1, \ldots,\lfloor N / 4\rfloor\}$, and defines the relation $R$ in such a way that, for $(x, i) \in X \times W$, we have $(x, i) \in R$ if and only if $x=g_{*}^{i}$. On the other hand, the subset sampling algorithm first chooses $\omega \in W$ uniformly at random, and then outputs $g_{*}^{\omega} \in L$ together with $\omega \in W$ as the witness. ${ }^{8}$ Now we have $|X|=p q p^{\prime} q^{\prime}$ and $\left|X^{\prime} \backslash L\right|=(p-1)(q-1) p^{\prime} q^{\prime}$, therefore $\left|X^{\prime} \backslash L\right| /|X|$ is overwhelming and the three uniform distributions on $X$, on $X \backslash L$ and on $X^{\prime} \backslash L$ have negligible statistical distances from each other. By this and Lemma 5.2, it follows that the construction of $\mathbf{M}$ satisfies the condition for a hard subset membership problem relative to $X^{\prime}$ provided the DDH assumption holds in $\mathbb{Z}_{N^{2}}^{*}$. Moreover, $X^{\prime} \backslash L$ is approximately samplable relative to $X$.

- In the trapdoor mode for $\mathbf{M}$, the algorithm also outputs $\tau:=p^{\prime} q^{\prime}$ as the trapdoor element. Then, by using $\tau$, it can be efficiently decided whether a given $x \in X$ is in $L$ or not, by checking if $x^{\tau}=1$. Hence, $\mathbf{M}$ is a hard trapdoor subset membership problem (relative to $X^{\prime}$ ).

- To define the corresponding diverse group system $\mathbf{G}$, we set $\Pi:=X$, and define $\mathcal{H}$ to be the set of homomorphisms $H_{k}: X \rightarrow \Pi$, indexed by $k \in K:=\mathbb{Z}_{p q p^{\prime} q^{\prime}}$, satisfying that $H_{k}(x):=x^{k}$ for any $x \in X$. Then $\mathbf{G}$ is diverse; indeed, for any $x \in X$, we have $H_{p^{\prime} q^{\prime}}(x)=x^{p^{\prime} q^{\prime}}=1$ if and only if $x \in L$.

\footnotetext{
${ }^{7}$ The probability that $g_{*}$ is not a generator of $L$ is $1-\left(1-1 / p^{\prime}\right)\left(1-1 / q^{\prime}\right)=1 / p^{\prime}+1 / q^{\prime}-1 /\left(p^{\prime} q^{\prime}\right)$, which is negligible (otherwise, the DCR assumption can be trivially broken since $\mathbb{Z}_{N^{2}}^{*}$ is not large enough).

${ }^{8}$ The distribution of the $g_{*}^{\omega}$ and the uniform distribution on $L$ have statistical distance $\left(\lfloor N / 4\rfloor-p^{\prime} q^{\prime}\right)\left(2 /\left(p^{\prime} q^{\prime}\right)-1 /\left(p^{\prime} q^{\prime}\right)\right) \leq$ $\left(2 p^{\prime}+1\right)\left(2 q^{\prime}+1\right) /\left(4 p^{\prime} q^{\prime}\right)-1=1 /\left(2 p^{\prime}\right)+1 /\left(2 q^{\prime}\right)+1 /\left(4 p^{\prime} q^{\prime}\right)$, which is negligible.
} 


\begin{tabular}{|c|c|}
\hline 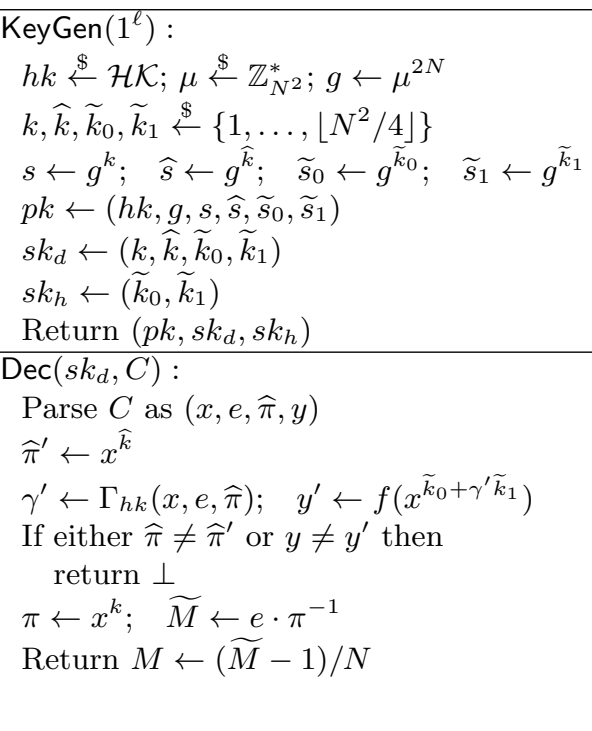 & 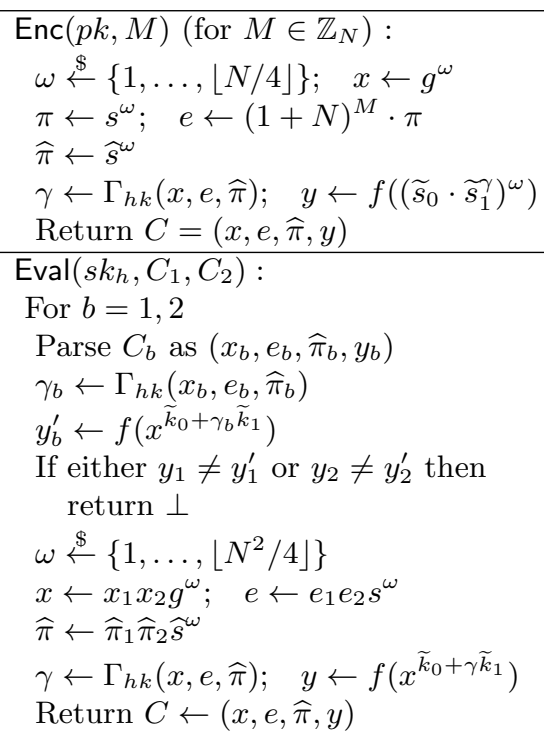 \\
\hline
\end{tabular}

Figure 3: DCR-based instantiation of our KH-PKE scheme (here $N=p q$ with $p=2 p^{\prime}+1$ and $q=2 q^{\prime}+1$ satisfies that the DCR assumption holds in $\mathbb{Z}_{N^{2}}^{*} ;\left\{\Gamma=\Gamma_{h k}: X^{3} \rightarrow\{0,1, \ldots, \widetilde{p}-1\} \mid h k \in \mathcal{H} \mathcal{K}\right\}$ is a CR hash family where $X=\left\{g^{2} \mid g \in \mathbb{Z}_{N^{2}}^{*}\right\}$ and $\widetilde{p}=\min \{p, q\} ;$ and $f: X \rightarrow \mathcal{Y}$ is a smooth function)

By the construction, we have $|X / L|=p q$ and $|\Pi|=p q p^{\prime} q^{\prime}$, therefore $\widetilde{p}=\min \{p, q\}$. This implies that, by setting both $\mathbf{P}$ and $\widehat{\mathbf{P}}$ to be the homomorphic HPS associated to $\mathbf{G}, \widehat{\mathbf{P}}$ is $(1 / \widetilde{p})$-universal by Lemma by 2.3 , and $1 / \widetilde{p}$ is negligible. On the other hand, for the HPS $\mathbf{P}$, we define the subgroup $\Pi^{\prime}$ of $\Pi=X$ by $\Pi^{\prime}:=\iota^{-1}\left(C_{p} \times 1 \times C_{q} \times 1\right)$. We note that $\Pi^{\prime}=\left\{x \in X \mid x^{N}=1\right\}$ and it is generated by $1+N \in \mathbb{Z}_{N^{2}}^{*}$, therefore a uniformly random element of $\Pi^{\prime}$ can be efficiently chosen (in particular, $\Pi^{\prime}$ is approximately samplable relative to $\Pi)$. Now we have the following:

Lemma 5.3. In the setting, $\mathbf{P}$ is 0 -smooth relative to $\left(X^{\prime}, \Pi^{\prime}\right)$.

Proof. Let $k \in K$ and $x \in X^{\prime} \backslash L$. Write $k=\lambda_{1} p^{\prime} q^{\prime}+\lambda_{2}$ with $\lambda_{1} \in\{0,1, \ldots, p q-1\}$ and $\lambda_{2} \in\left\{0,1, \ldots, p^{\prime} q^{\prime}-\right.$ $1\}$, and $\iota(x)=y=\left(y_{p}, y_{p^{\prime}}, y_{q}, y_{q^{\prime}}\right)$. Then we have $y_{p} \neq 1$ and $y_{q} \neq 1$ by the definition of $X^{\prime}$ and $L$. Put $y_{p, q}:=\left(y_{p}, 1, p_{q}, 1\right)$ and $y_{p^{\prime}, q^{\prime}}:=\left(1, y_{p^{\prime}}, 1, y_{q^{\prime}}\right)$. On the other hand, we have $s=\alpha(k)=g_{*}^{k}=g_{*}^{\lambda_{2}}$ since $g_{*} \in L$, therefore $\lambda_{2}$ is uniquely determined from $s$ since $g_{*}$ is a generator of $L$. Now we have

$$
y^{k}=y^{\lambda_{1} p^{\prime} q^{\prime}} y^{\lambda_{2}}=y_{p, q}^{\lambda_{1} p^{\prime} q^{\prime}} y_{p^{\prime}, q^{\prime}}^{\lambda_{1} p^{\prime} q^{\prime}} y^{\lambda_{2}}=\left(y_{p, q}^{p^{\prime} q^{\prime}}\right)^{\lambda_{1}} y^{\lambda_{2}}
$$

therefore $x^{k}=\iota^{-1}\left(y_{p, q}^{p^{\prime} q^{\prime}}\right)^{\lambda_{1}} x^{\lambda_{2}}$. Since $y_{p} \neq 1$ and $y_{q} \neq 1, y_{p, q}^{p^{\prime} q^{\prime}}$ is a generator of $C_{p} \times 1 \times C_{q} \times 1$. Hence, when $k$ is chosen uniformly at random subject to the condition $\alpha(k)=s$ for a given $s, \lambda_{1}$ is uniformly random while $\lambda_{2}$ is fixed, therefore $x^{k}$ is the product of the fixed element $x^{\lambda_{2}}$ of $\Pi$ and a uniformly random element $\iota^{-1}\left(y_{p, q}^{p^{\prime} q^{\prime}}\right)^{\lambda_{1}}$ of $\Pi^{\prime}$. This implies that $\mathbf{P}$ is 0 -smooth relative to $\left(X^{\prime}, \Pi^{\prime}\right)$, as desired.

Moreover, let $\Gamma=\Gamma_{h k}: X \times \Pi^{2} \rightarrow\{0,1, \ldots, \widetilde{p}-1\}$ be a function indexed by $h k \in \mathcal{H} \mathcal{K}$ sampled from a

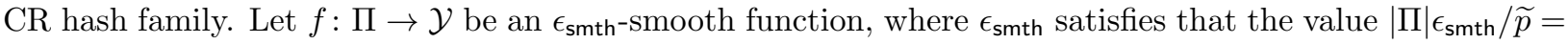
$p q p^{\prime} q^{\prime} \epsilon_{\text {smth }} / \min \{p, q\}$ is negligible (for example, $f$ may be an identity mapping $\Pi \rightarrow \Pi$; then we have $\epsilon_{\text {smth }}=1 /|\Pi|$ and $|\Pi| \epsilon_{\text {smth }} / \widetilde{p}=1 / \widetilde{p}$ is negligible, as desired). Then, since $\mathbf{M}$ is a trapdoor subset membership problem, Proposition 5.1 implies that the composition (denoted by $\widetilde{\mathbf{P}}$ ) of $f$ and the CS hash proof system constructed from the diverse group system $\mathbf{G}$ and the internal function $\Gamma$ is a first-adaptive computationally universal ${ }_{2}$ hash proof system.

Summarizing, all the conditions for Theorem 4.1 with Assumption A are satisfied, therefore the resulting instantiation of our KH-PKE scheme is KH-CCA secure. We write down the instantiation of the KHPKE scheme in Figure 3. Here we note that, for the choice of secret keys for the hash proof systems, the 
uniform distribution on $\left\{1, \ldots, p q p^{\prime} q^{\prime}\right\}$ has negligible statistical distance from the uniform distribution on $\left\{1, \ldots,\left\lfloor N^{2} / 4\right\rfloor\right\}$. We note also that, the multiplicative group $\Pi^{\prime}$ is isomorphic to the additive group $\mathbb{Z}_{N}$, with efficiently computable isomorphism $\mathbb{Z}_{N} \ni M \mapsto(1+N)^{M} \bmod N^{2} \in \Pi^{\prime}$ and its efficiently computable inverse $\Pi^{\prime} \ni \widetilde{M} \mapsto(\widetilde{M}-1) / N \bmod N \in \mathbb{Z}_{N}$. In the instantiation here, we switch the plaintext space from $\Pi^{\prime}$ to $\mathbb{Z}_{N}$ via the isomorphism. As in [15], we implicitly assume that the Dec algorithm checks that $x$, $e$, and $\widehat{\pi}$ lie in $\mathbb{Z}_{N^{2}}^{*}$ and $\widetilde{M}-1$ is a multiple of $N$.

\section{Keyed-Homomorphic Identity-Based Encryption}

In this section, we give a formal definition of KH-IBE and its concrete construction from the Gentry IBE scheme. In KH-IBE, one can perform the homomorphic operation to ciphertexts if these ciphertexts are generated by the same identity and one has a homomorphic operation key. As a different point from KHPKE, a homomorphic operation key is generated for each identity. Thus, CCA security is guaranteed for one who does not have the corresponding homomorphic operation key.

\subsection{Syntax of KH-IBE}

Definition 6.1 (Syntax of KH-IBE for homomorphic operation $\odot$ ). Let $\mathcal{M}$ be a message space, $\mathcal{I D}$ be an identity space, and $\odot$ be a binary operation over $\mathcal{M}$. A KH-IBE scheme $\mathcal{K} \mathcal{H}$-IBE $=($ IBE.Setup, IBE.KeyGen, IBE.Enc, IBE.Dec, IBE.Eval) consists of the following five algorithms:

IBE.Setup: This algorithm takes a security parameter $1^{\ell}(\ell \in \mathbb{N})$ as input, and returns a public parameter params and a master secret key msk.

IBE.KeyGen: This algorithm takas params, msk, and an identity ID $\in \mathcal{I D}$ as input, and returns a decryption key $s k_{d, \mathrm{ID}}$ and a homomorphic operation key $s k_{h, \mathrm{ID}} \cdot{ }^{9}$

IBE.Enc: This algorithm takes params, ID, and a message $M \in \mathcal{M}$ as input, and returns a ciphertext $C$.

IBE.Dec: This algorithm takes params, sk $k_{d, \mathrm{ID}}$ and $C$ as input, and returns $M$ or $\perp$.

IBE.Eval: This algorithm takes params, sk $k_{h, \mathrm{ID}}$ and two ciphertexts $C_{1}$ and $C_{2}$ as input, and returns a ciphertext $C$ or $\perp$.

Let ID $\in \mathcal{I D}$ be an identity, params be a public parameter generated by the IBE.Setup, and $\mathcal{C}_{\mathrm{ID}, M}$ be the set of all ciphertexts of $M \in \mathcal{M}$ under the public key $I D$, i.e., $\mathcal{C}_{\mathrm{ID}, M}=\left\{C \mid \exists r \in\{0,1\}^{*}\right.$ s.t. $C=$ IBE.Enc(params, ID, $M ; r)\}$.

Definition 6.2 (Correctness). A KH-IBE scheme for homomorphic operation $\odot$ is said to be correct if for all (params, msk $) \leftarrow$ IBE.Setup $\left(1^{k}\right)$, (1) for all ID $\in \mathcal{I D}$ and $\left(s k_{d, \mathrm{ID}}, s k_{h, \mathrm{ID}}\right) \leftarrow \mathrm{IBE}$.KeyGen $($ params, msk, ID $)$, all $M \in \mathcal{M}$, and all $C \in \mathcal{C}_{\mathrm{ID}, M}$, it holds that IBE.Dec(params, sk $\left.k_{d, \mathrm{ID}}, C\right)=M$. (2) For all ID $\in \mathcal{I D}$ and all $\left(s k_{d, \mathrm{ID}}, s k_{h, \mathrm{ID}}\right) \leftarrow \mathrm{IBE} . K e y G e n($ params, msk, ID $)$, all $M_{1}, M_{2} \in \mathcal{M}$, all $C_{1} \in \mathcal{C}_{\mathrm{ID}, M_{1}}$ and $C_{2} \in \mathcal{C}_{\mathrm{ID}, M_{2}}$, it holds that IBE.Eval(params, $\left.s k_{h, \mathrm{ID}}, C_{1}, C_{2}\right) \in \mathcal{C}_{\mathrm{ID}, M_{1} \odot M_{2}}$.

Next, we define the security notion for KH-IBE, which we call indistinguishability of message under adaptive chosen ciphertext and identity attacks (KH-ID-CCA).

Definition 6.3 (KH-ID-CCA). A KH-IBE scheme is said to be KH-ID-CCA secure if for any PPT adversary $\mathcal{A}$, the advantage

\footnotetext{
${ }^{9}$ In this paper, we assume that the IBE.KeyGen algorithm is deterministic as in the definition of the Gentry IBE.
} 


$$
\begin{gathered}
A d v_{\mathrm{KH}-\mathrm{IBE}, \mathcal{A}}^{\mathrm{KH} C \mathrm{ID} C A}(\ell)=\mid \operatorname{Pr}\left[(\text { params }, \text { msk }) \leftarrow \operatorname{IBE} . \operatorname{Setup}\left(1^{k}\right) ;\right. \\
\left(M_{0}^{*}, M_{1}^{*}, \mathrm{ID}^{*}, \text { State }\right) \leftarrow \mathcal{A}^{\mathcal{O}}(\text { find } \text { params }) ; \\
\beta \stackrel{\$}{\leftarrow}\{0,1\} ; C^{*} \leftarrow \mathrm{IBE} . \operatorname{Enc}\left(\text { params, ID }, M_{\beta}^{*}\right) ; \\
\left.\left.\beta^{\prime} \leftarrow \mathcal{A}^{\mathcal{O}} \text { (guess, State, } C^{*}\right): \beta=\beta^{\prime}\right]-\frac{1}{2} \mid
\end{gathered}
$$

is negligible in $\ell$, where $\mathcal{O}$ consists of the four oracles $\mathcal{E} v a l(\cdot, \cdot, \cdot), \mathcal{R} \operatorname{RevDK}(\cdot), \mathcal{R} \operatorname{evH} K(\cdot)$, and $\operatorname{Dec}(\cdot, \cdot)$ defined as follows. Let $\mathcal{D}$ be a list which is set as $\mathcal{D}=\left\{C^{*}\right\}$ right after the challenge stage $(\mathcal{D}$ is set as $\emptyset$ in the find stage).

- The evaluation oracle $\mathcal{E}$ val $(\cdot, \cdot, \cdot)$ : This oracle responds to a query $\left(\mathrm{ID}, C_{1}, C_{2}\right)$ with the result of $C=$ IBE.Eval $\left(s k_{h, \mathrm{ID}}, C_{1}, C_{2}\right)$. In addition, in the case ID $=\mathrm{ID}^{*}$, if $C \neq \perp$ and either $C_{1} \in \mathcal{D}$ or $C_{2} \in \mathcal{D}$, then the oracle updates the list by $\mathcal{D} \leftarrow \mathcal{D} \cup\{C\}$.

- The key generation oracle $\mathcal{R} e v D K(\cdot)$ : This oracle responds to a query ID $\in \mathcal{I D}$ with sk $k_{d, \mathrm{ID}}$ where $s k_{d, \mathrm{ID}}$ is the result of $\left(s k_{d, \mathrm{ID}}, s k_{h, \mathrm{ID}}\right) \leftarrow \mathrm{IBE} . \mathrm{KeyGen}($ params, msk, ID). $\mathcal{A}$ is not allowed to query ID* to the oracle.

- The homomorphic key reveal oracle $\mathcal{R} e v H K(\cdot)$ : This oracle responds to a query ID $\in \mathcal{I D}$ with $s k_{h, \mathrm{ID}}$ where $s k_{h, \mathrm{ID}}$ is the result of $\left(s k_{d, \mathrm{ID}}, s k_{h, \mathrm{ID}}\right) \leftarrow \mathrm{IBE} . \mathrm{KeyGen}($ params, msk, ID $)$.

- The decryption oracle Dec $(\cdot, \cdot)$ : For a query $(\mathrm{ID}, C)$ and $\mathrm{ID}=\mathrm{ID}^{*}$, this oracle is not available if $\mathcal{A}$ has sent ID* to $\mathcal{R e v H K}$ (i.e, $\mathcal{A}$ has obtained $\left.s k_{h, \mathrm{ID}^{*}}\right)$ and $\mathcal{A}$ has obtained the challenge ciphertext $C^{*}$. Otherwise, this oracle responds to a query $C$ with the result of $\mathrm{IBE} \cdot \operatorname{Dec}\left(s k_{d, \mathrm{ID}}, C\right)$ if $C \notin \mathcal{D}$ or ID $\neq \mathrm{ID}^{*}$, or returns $\perp$ if $C \in \mathcal{D}$ and $\mathrm{ID}=\mathrm{ID}^{*}$.

\subsection{Proposed KH-IBE Scheme}

In Figure 4 , we give our proposed KH-IBE scheme. Here, $\left(c_{1}, c_{2}, c_{3}, c_{5}\right)$ is essentially the same as a ciphertext of the original (CCA secure) Gentry IBE scheme, and $\left(c_{1}, c_{2}, c_{3}, c_{4}\right)$ is its CCA1 secure and homomorphic variant. As in our KH-PKE schemes, $c_{5}$ in the ciphertext of our KH-IBE for validity checking upon the homomorphic operation, and this can be compressed into a smaller value $\tau$ due to the smoothness of the function $f$.

Our KH-IBE scheme is comparably efficient to the original Gentry IBE, and secure under the truncated decisional augmented bilinear Diffie-Hellman exponent (truncated decisional ABDHE) assumption as in the original Gentry IBE, which is defined as follows.

Definition 6.4 (truncated decision $q$-ABDHE $[22]$ ). Let $\mathbb{G}$ and $\mathbb{G}_{T}$ be cyclic groups with prime order $p$, where $\langle g\rangle=\mathbb{G}$, and $e: \mathbb{G} \times \mathbb{G} \rightarrow \mathbb{G}_{T}$ be a bilinear map. Let $g^{\prime} \stackrel{\mathbb{S}}{\leftarrow}, \alpha \stackrel{\mathbb{S}}{\leftarrow} \mathbb{Z}_{p}$, and $Z \stackrel{\mathbb{\&}}{\leftarrow} \mathbb{G}_{T}$, and set $g_{i}^{\prime}:=g^{\prime\left(\alpha^{i}\right)}$ and $g_{i}:=g^{\left(\alpha^{i}\right)}$. We say that truncated decision $q-A B D H E$ assumption holds, if for any PPT adversary $\mathcal{A}$, its advantage $\operatorname{Adv}_{\mathcal{A}}^{\mathrm{ABDHE}}(\ell)$ defined by $\operatorname{Adv}_{\mathcal{A}}^{\mathrm{ABDHE}}(\ell):=\mid \operatorname{Pr}\left[\mathcal{A}\left(g^{\prime}, g_{q+2}^{\prime}, g, g_{1}, \ldots, g_{q}, e\left(g_{q+1}, g^{\prime}\right)\right)=0\right]-$ $\operatorname{Pr}\left[\mathcal{A}\left(g^{\prime}, g_{q+2}^{\prime}, g, g_{1}, \ldots, g_{q}, Z\right)=0\right] \mid$ is negligible

Theorem 6.1. Our construction above is KH-ID-CCA-secure, if truncated decision $q$-ABDHE assumption holds and $\Gamma_{h k}$ is a TCR hash family. Here, $q:=q_{\mathrm{ID}}+2$ and $q_{\mathrm{ID}}$ is the number of key generation queries.

Before starting the security proof of 6.1, we show that the following property holds as in the KH-PKE case. We say that a ciphertext $C$ is $\tau$-consistent if $\tau=f\left(e\left(c_{1}, h_{\mathrm{ID}, 3} h_{\mathrm{ID}, 4}^{\delta}\right) c_{2}^{r_{\mathrm{ID}, 3}+r_{\mathrm{I}, 4} \delta}\right)$.

Lemma 6.1 (Source Ciphertext Hiding Property). Let (params, msk) $\leftarrow$ IBE.Setup $\left(1^{k}\right)$. For all ID $\in \mathcal{I D}$, let $\left(s k_{d, \mathrm{ID}}, s k_{h, \mathrm{ID}}\right) \leftarrow \mathrm{IBE} . \mathrm{KeyGen}($ params, msk, ID $)$, and let $C_{2}=\left(c_{2,1}, c_{2,2}, c_{2,3}, c_{2,4}, \tau_{2}\right)$ be any $\tau$-consistent 


\begin{tabular}{|c|c|}
\hline $\begin{array}{l}\text { IBE.Setup }\left(1^{\ell}\right): \\
h k \stackrel{\$}{\leftarrow} \mathcal{H} \mathcal{K} ; g \stackrel{\$}{\leftarrow} \mathbb{G} \\
h_{i} \stackrel{\$}{\leftarrow} \mathbb{G} \text { for } i \in[4] \\
\alpha \stackrel{\$}{\leftarrow} \mathbb{Z}_{p} ; \quad g_{1} \leftarrow g^{\alpha} \\
\text { Return } \\
\quad \text { params } \leftarrow\left(g, g_{1}, h_{1}, h_{2}, h_{3}, h_{4}, h k, f\right) \\
\quad \text { and } m s k \leftarrow \alpha \\
\text { IBE.KeyGen }(\text { params, msk, ID }): \\
\text { For } i \in[4] \\
r_{I D, i} \stackrel{\$}{\leftarrow} \mathbb{Z}_{p} \\
h_{I D, i} \leftarrow\left(h_{i} g^{-r_{I D, i}}\right)^{1 /(\alpha-I D)} \\
\text { Return } s k_{d, \mathrm{ID}}=\left\{\left(r_{\mathrm{ID}, i}, h_{\mathrm{ID}, i}\right)\right\}_{i=1}^{4} \\
\quad \text { and } s k_{h, \mathrm{ID}}=\left\{\left(r_{\mathrm{ID}, i}, h_{\mathrm{ID}, i}\right)\right\}_{i=3}^{4} \\
\mathrm{IBE} \text { Dec }\left(\text { params }_{i} C, \mathrm{dk} \mathrm{k}_{\mathrm{ID}}\right): \\
\text { Parse } s k_{d, I D} \text { as }\left\{\left(r_{I D, i}, h_{I D, i}\right)\right\}_{i=1}^{4} \\
\text { Parse } C \text { as }\left(c_{1}, c_{2}, c_{3}, c_{4}, \tau\right) \\
\delta \leftarrow \Gamma_{h k}\left(c_{1}, c_{2}, c_{3}, c_{4}\right) \\
c_{4}^{\prime} \leftarrow e\left(c_{1}, h_{\mathrm{ID}, 2}\right) c_{2}^{r_{\mathrm{D}}, 2} \\
c_{5} \leftarrow e\left(c_{1}, h_{\mathrm{ID}, 3} h_{\mathrm{ID}, 4}^{\delta}\right) c_{2}^{r_{\mathrm{II}, 3}+r_{\mathrm{ID}, 4} \delta} \\
\text { If } c_{4}^{\prime} \neq c_{4} \text { or } \tau \neq f\left(c_{5}\right) \text { then return } \perp \\
\text { Return } M \leftarrow c_{3} \cdot e\left(c_{1}, h_{\mathrm{ID}, 1}\right) c_{2}^{r_{\mathrm{ID}, 1}}\end{array}$ & 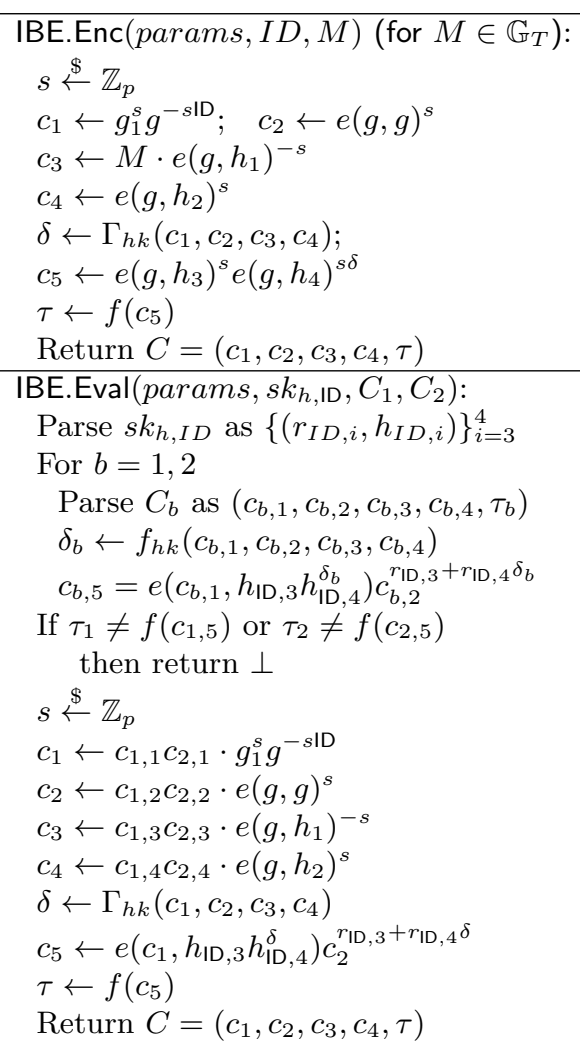 \\
\hline
\end{tabular}

Figure 4: Our KH-IBE scheme. Here $\mathbb{G}$ and $\mathbb{G}_{T}$ are groups of prime order $p ; e: \mathbb{G} \times \mathbb{G} \rightarrow \mathbb{G}_{T}$ is a bilinear map; $\mathcal{I D}:=\mathbb{Z}_{p}$ is an identity space; $\mathcal{M}:=\mathbb{G}_{T}$ is a message space; $\left\{\Gamma=\Gamma_{h k}: \mathbb{G}^{4} \rightarrow\{0,1, \ldots, p-1\} \mid h k \in \mathcal{H} \mathcal{K}\right\}$ is a TCR hash family; and $f: \mathbb{G}_{T} \rightarrow \mathcal{Y}$ is a smooth function.

ciphertext, and assume that ciphertexts $C_{1}=\left(c_{1,1}, c_{1,2}, c_{1,3}, c_{1,4}, \tau_{1}\right)$ and $C_{1}^{\prime}=\left(c_{1,1}^{\prime}, c_{1,2}^{\prime}, c_{1,3}^{\prime}, c_{1,4}^{\prime}, \tau_{1}^{\prime}\right)$ are $\tau$-consistent and satisfy the following conditions: for some $s \in \mathbb{Z}_{p}$,

$$
\begin{array}{r}
c_{1}^{\prime \prime}:=c_{1,1}^{\prime} / c_{1,1}=g_{1}^{s} g^{-s I \mathrm{D}}, c_{2}^{\prime \prime}:=c_{1,2}^{\prime} / c_{1,2}=e(g, g)^{s}, \\
c_{3}^{\prime \prime}:=c_{1,3}^{\prime} / c_{1,3}=e\left(g, h_{1}\right)^{-s}, \text { and } c_{4}^{\prime \prime}:=c_{1,4}^{\prime} / c_{1,4}=e\left(g, h_{2}\right)^{-s} .
\end{array}
$$

As a remark, $c_{1}^{\prime \prime}:=c_{1,1}^{\prime} / c_{1,1}=g_{1}^{s} g^{-s \mathrm{ID}}$ means $C_{1}$ and $C_{1}^{\prime}$ are ciphertexts of the same identity ID, and $c_{3}^{\prime}:=c_{1,3}^{\prime} / c_{1,3}=e\left(g, h_{1}\right)^{-s}$ means $C_{1}$ and $C_{1}^{\prime}$ are ciphertexts of the same plaintext. Then the outputs of IBE.Eval (params, sk $\left.k_{h, \mathrm{ID}}, C_{1}, C_{2}\right)$ and of IBE.Eval(params, sk $\left.k_{h, \mathrm{ID}}, C_{1}^{\prime}, C_{2}\right)$ also satisfy the condition in (2), and the distributions of these two outputs of IBE.Eval are identical.

Proof. The first four components of IBE.Eval(params, $\left.s k_{h, \mathrm{ID}}, C_{1}, C_{2}\right)$ are of the from $c_{1}:=c_{1,1} c_{2,1} \cdot g_{1}^{s^{\prime}} g^{-s^{\prime} \mathrm{ID}}$, $c_{2}:=c_{1,2} c_{2,2} \cdot e(g, g)^{s^{\prime}}, c_{3}:=c_{1,3} c_{2,3} \cdot e\left(g, h_{1}\right)^{-s^{\prime}}$, and $c_{4}:=c_{1,4} c_{2,4} \cdot e\left(g, h_{2}\right)^{s^{\prime}}$ with $s^{\prime} \stackrel{\$}{\leftarrow} \mathbb{Z}_{p}$, and the first four components of IBE.Eval (params, $\left.s k_{h, \mathrm{ID}}, C_{1}^{\prime}, C_{2}\right)$ are of the from $c_{1}^{\prime}:=c_{1,1}^{\prime} c_{2,1} \cdot g_{1}^{s^{\prime \prime}} g^{-s^{\prime \prime I D}}, c_{2}^{\prime}:=$ $c_{1,2}^{\prime} c_{2,2} \cdot e(g, g)^{s^{\prime \prime}}, c_{3}^{\prime}:=c_{1,3}^{\prime} c_{2,3} \cdot e\left(g, h_{1}\right)^{-s^{\prime \prime}}$, and $c_{4}^{\prime}:=c_{1,4}^{\prime} c_{2,4} \cdot e\left(g, h_{2}\right)^{s^{\prime \prime}}$ with $s^{\prime \prime} \stackrel{\$}{\leftarrow} \mathbb{Z}_{p}$. Now we have

$$
c_{1}^{\prime}=c_{1,1}^{\prime} c_{2,1} \cdot g_{1}^{s^{\prime \prime}} g^{-s^{\prime \prime} \mathrm{ID}}=c_{1}^{\prime \prime} c_{1,1} c_{2,1} \cdot g_{1}^{s^{\prime \prime}} g^{-s^{\prime \prime} \mathrm{ID}}=c_{1}^{\prime \prime} c_{1} \cdot g_{1}^{s^{\prime \prime}-s^{\prime}} g^{-\left(s^{\prime \prime}-s^{\prime}\right) \mathrm{ID}} \text {. }
$$


where $c_{1}^{\prime \prime}:=c_{1,1}^{\prime} / c_{1,1}=g_{1}^{s} g^{-s \mathrm{ID}}$. Similarly, due to the homomorphic property, we have

$$
\begin{aligned}
& c_{2}^{\prime}:=c_{1,2}^{\prime} c_{2,2} \cdot e(g, g)^{s^{\prime \prime}}=c_{2}^{\prime \prime} c_{1,2} c_{2,2} \cdot e(g, g)^{s^{\prime \prime}}=c_{2}^{\prime \prime} c_{2} \cdot e(g, g)^{s^{\prime \prime}-s^{\prime}}, \\
& c_{3}^{\prime}:=c_{1,3}^{\prime} c_{2,3} \cdot e\left(g, h_{1}\right)^{-s^{\prime \prime}}=c_{3}^{\prime \prime} c_{1,3} c_{2,3} \cdot e\left(g, h_{1}\right)^{-s^{\prime \prime}}=c_{3}^{\prime \prime} c_{3} \cdot e\left(g, h_{1}\right)^{-\left(s^{\prime \prime}-s^{\prime}\right)}, \\
& c_{4}^{\prime}:=c_{1,4}^{\prime} c_{2,4} \cdot e\left(g, h_{2}\right)^{s^{\prime \prime}}=c_{4}^{\prime \prime} c_{1,4} c_{2,4} \cdot e\left(g, h_{2}\right)^{s^{\prime \prime}}=c_{4}^{\prime \prime} c_{4} \cdot e\left(g, h_{2}\right)^{s^{\prime \prime}-s^{\prime}}
\end{aligned}
$$

Since $c_{1}^{\prime \prime}$ has the form $g_{1}^{s} g^{-s I D}$ for some $s \in \mathbb{Z}_{p}, c_{1}^{\prime \prime} g_{1}^{s^{\prime \prime}-s^{\prime}} g^{-\left(s^{\prime \prime}-s^{\prime}\right) \text { ID }}$ also has the same form such as

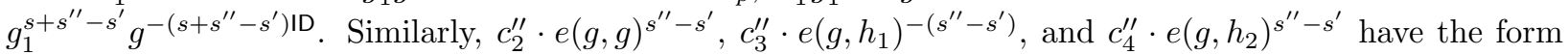
$e(g, g)^{s+s^{\prime \prime}-s^{\prime}}, e\left(g, h_{1}\right)^{-\left(s+s^{\prime \prime}-s^{\prime}\right)}$, and $e\left(g, h_{2}\right)^{s+s^{\prime \prime}-s^{\prime}}$, respectively. Therefore the condition in (2) is satisfied. Moreover, since $s^{\prime}, s^{\prime \prime} \stackrel{\mathbb{S}}{\leftarrow} \mathbb{Z}_{p}$, the distributions of $\left(c_{1}, c_{2}, c_{3}, c_{4}\right)$ and $\left(c_{1}^{\prime}, c_{2}^{\prime}, c_{3}^{\prime}, c_{4}^{\prime}\right)$ are identical. Hence, the claim holds.

We start the proof of Theorem 6.1. Basically, this is the same as that of the proof of 4.1, where the proof is divided as three parts, preliminary part, main part, and concluding part.

Proof of Theorem 6.1. Let $\mathcal{A}$ be a PPT adversary against the KH-ID-CCA security of our construction. Our goal in the proof is to show that the advantage $A d v_{\text {KH-IBE. } \mathcal{A}}^{K H C A}(\ell)$ of $\mathcal{A}$ is negligible. First note that, since $\mathcal{A}$ is of polynomial time, there exists a polynomial $Q(\ell)$ with the property that the total number of decryption queries, key generation queries, and evaluation queries made by $\mathcal{A}$ is not larger than $Q(\ell)$ for any security parameter $\ell$.

Preliminary part of the game-hopping: Let $T_{\ell}^{(i)}$ denote the event that Game $i$ outputs 1 .

Game pre-0. Let $C^{*}=\left(c_{1}^{*}, c_{2}^{*}, c_{3}^{*}, c_{4}^{*}, \tau^{*}\right)$ be the challenge ciphertext where $C^{*} \leftarrow \operatorname{IBE}$. Enc $\left(\right.$ params, ID*,$\left.M_{\beta}^{*}\right)$. If $\mathcal{A}$ outputs $\beta^{\prime}=\beta$, then this game outputs 1 , and 0 otherwise. Then, $\left|\operatorname{Pr}\left[T_{\ell}^{(\text {pre-0) }}\right]-1 / 2\right|=A d v_{\mathrm{KH}-\mathrm{IBE}, \mathcal{A}}^{\mathrm{KH}-\mathrm{IDCA}}(\ell)$ holds.

Game pre-1. In comparison to Game pre-0, in guess stage, we introduce another auxiliary dictionary $\mathcal{D}^{\prime}$ and modify the rule for the challenger to reply evaluation queries (ID, $C^{\prime}, C^{\prime \prime}$ ) satisfying that ID $=I^{*}$ and at least one of $C^{\prime}$ and $C^{\prime \prime}$ is listed in the original dictionary $\mathcal{D}$ and the query is not rejected. When $\mathcal{D}=\left(C_{0}, C_{1}, \ldots, C_{\kappa}\right)$ where $C_{0}=C^{*}$ and $C_{1}, \ldots, C_{\kappa}$ were added to $\mathcal{D}$ in this order, $\mathcal{D}^{\prime}$ is of the form $\left(\left(D_{1}^{\prime}, D_{1}^{\prime \prime}\right),\left(D_{2}^{\prime}, D_{2}^{\prime \prime}\right), \ldots,\left(D_{\kappa}^{\prime}, D_{\kappa}^{\prime \prime}\right)\right)$ where each of $D_{i}^{\prime}$ and $D_{i}^{\prime \prime}(i \in[1, \kappa])$ is either a ciphertext with fifth component being consistent or an index in $\{0,1, \ldots, i-1\}$. Intuitively, the content of $\mathcal{D}^{\prime}$ means that $C_{i}$ was the reply to the evaluation query $\left(D_{i}^{\prime}, D_{i}^{\prime \prime}\right)$ where, if $D_{i}^{\prime}$ or $D_{i}^{\prime \prime}$ is an index $j$, then it is interpreted as $C_{j}$.

Now, we describe the modified rule for the challenger to reply $(\kappa+1)$-th evaluation queries (ID, $\left.C^{\prime}, C^{\prime \prime}\right)$ as above, where $\mathcal{D}=\left(C_{0}, C_{1}, \ldots, C_{\kappa}\right)$ and $\mathcal{D}^{\prime}=\left(\left(D_{1}^{\prime}, D_{1}^{\prime \prime}\right),\left(D_{2}^{\prime}, D_{2}^{\prime \prime}\right), \ldots,\left(D_{\kappa}^{\prime}, D_{\kappa}^{\prime \prime}\right)\right)$. We call it the $(\kappa+1)$-th refreshing process in the sequel, and we also call the query (ID, $\left.C^{\prime}, C^{\prime \prime}\right)$ the $(\kappa+1)$-th refreshing query. In the process, the challenger first generates auxiliary ciphertexts $\bar{C}_{0}{ }^{(\kappa+1)}=\bar{C}^{*}(\kappa+1), \bar{C}_{1}{ }^{(\kappa+1)}, \ldots, \bar{C}_{\kappa}{ }^{(\kappa+1)}$ as follows:

- The challenger generates $\bar{C}^{*}(\kappa+1) \leftarrow \operatorname{IBE} . \operatorname{Enc}\left(\right.$ params, ID*,$\left.M_{\beta}^{*}\right)$ instead of using $C^{*}$ itself, which we call the source ciphertext for the refreshing process.

- For each $i=1,2, \ldots, \kappa$, the challenger generates $\bar{C}_{i}{ }^{(\kappa+1)}$ by using the algorithm IBE.Eval, where its third (respectively, fourth) input is $D_{i}^{\prime}$ (respectively $D_{i}^{\prime \prime}$ ) if $D_{i}^{\prime}$ (respectively $D_{i}^{\prime \prime}$ ) is a ciphertext (i.e., not an index), and it is $\bar{C}_{j}^{(\kappa+1)}$ if $D_{i}^{\prime}$ (respectively $D_{i}^{\prime \prime}$ ) is an index $j \in\{0,1, \ldots, i-1\}$.

Secondly, the challenger sets $D_{\kappa+1}^{\prime}:=C^{\prime}$ if $C^{\prime} \notin \mathcal{D}$, and $D_{\kappa+1}^{\prime}:=i$ if $C^{\prime} \in \mathcal{D}$ and $i$ is the smallest index satisfying $C^{\prime}=C_{i} \in \mathcal{D}$. The challenger also determines $D_{\kappa+1}^{\prime \prime}$ similarly by using $C^{\prime \prime}$ instead of $C^{\prime}$. Thirdly, the challenger generates $C_{\kappa+1}$ by using the algorithm IBE. Eval, where its third (respectively, fourth) input is $D_{\kappa+1}^{\prime}$ (respectively, $D_{\kappa+1}^{\prime \prime}$ ) if $D_{\kappa+1}^{\prime}$ (respectively, $D_{\kappa+1}^{\prime \prime}$ ) is a ciphertext, and it is $\bar{C}_{i}{ }^{(\kappa+1)}$ if $D_{\kappa+1}^{\prime}$ (respectively, $\left.D_{\kappa+1}^{\prime \prime}\right)$ is an index $i \in\{0,1, \ldots, \kappa\}$. Finally, the challenger adds $C_{\kappa+1}$ to $\mathcal{D}$, adds $\left(D_{\kappa+1}^{\prime}, D_{\kappa+1}^{\prime \prime}\right)$ to $\mathcal{D}^{\prime}$, and gives $C_{\kappa+1}$ to the adversary as the reply to the evaluation query. 
By the source ciphertext hiding property, the distributions of $\bar{C}_{1}^{(\kappa+1)}, \ldots, \bar{C}_{\kappa}^{(\kappa+1)}$ are identical to those of $C_{1}, \ldots, C_{\kappa}$. Therefore, by the source ciphertext hiding property again, the distribution of $C_{\kappa+1}$ in the modified rule is identical to that of $C_{\kappa+1}$ in the original rule. This implies that the distribution of the adversary's view is identical in the two cases, therefore we have $\operatorname{Pr}\left[T_{\ell}^{(\text {pre-1) }}\right]=\operatorname{Pr}\left[T_{\ell}^{(\operatorname{pre}-0)}\right]$.

Main part of the game-hopping: From now, we proceed the game-hopping to remove the information on $\beta$ from the source ciphertexts one by one. The process is performed by the following sequence of Games $0,1, \ldots, Q(\ell)$, where Game 0 is identical to Game pre-1:

Game $\kappa(1 \leq \kappa \leq Q(\ell))$. In Game $\kappa$, a random message $M \in \mathbb{G}_{T}$ is used for the $\kappa^{\prime}$-th evaluation query instead of using $M_{\beta}^{*}$, where $1 \leq \kappa^{\prime} \leq \kappa$. From now on, we construct an algorithm $\mathcal{B}$ that solves the truncated decision $q$-ABDHE problem by using whether $\mathcal{A}$ can distinguish $\beta$ or not, where $q=Q(\ell)$.

Lemma 6.2. There exist PPT algorithms $\mathcal{B}_{1}$ and $\mathcal{B}_{2}$ which satisfy $\operatorname{Pr}\left[T_{\ell}^{(\kappa)}\right]-\operatorname{Pr}\left[T_{\ell}^{(\kappa-1)}\right] \leq 2\left(A d v_{\mathcal{B}_{1}}^{\mathrm{ABDHE}}(\ell)+\right.$ $\left.A d v_{\mathcal{B}_{2}}^{\mathrm{TCR}}(\ell)+\epsilon_{\mathrm{Smth}}+Q(\ell) / p\right)$.

Let define the following subGames from $\kappa .0$ to $\kappa .2$. We say that a ciphertext is irregular, if $c_{2} \neq$ $e\left(c_{1}, g\right)^{1 /(\alpha-\mathrm{ID})}$, and regular otherwise.

Game $\kappa .0$ Same as Game $\kappa$.

Game $\kappa .1$ If an irregular ciphertext which is not contained in $\mathcal{D}$ is queried to the decryption oracle or the evaluation oracle, then reject this query. Let $\bar{C}^{*}(\kappa)$ be an irregular source ciphertext of a random plaintext.

Claim 6.1. There exist PPT algorithms $\mathcal{B}_{1}$ and $\mathcal{B}_{2}$ that satisfy $\operatorname{Pr}\left[T_{\ell}^{(\kappa .0)}\right]-\operatorname{Pr}\left[T_{\ell}^{(\kappa \cdot 1)}\right] \leq A d v_{\mathcal{B}_{1}}^{\mathrm{ABDHE}}(\ell)+$ $A d v_{\mathcal{B}_{2}}^{\mathrm{TCR}}(\ell)+\epsilon_{\mathrm{Smth}}+Q(\ell) / p$.

Proof. Let $\left(g^{\prime}, g_{q+2}^{\prime}, g, g_{1}, \ldots, g_{q}, Z\right)$ be an instance of the truncated decision $q$-ABDHE problem where $q=$ $Q(\ell)$. $\mathcal{B}_{1}$ chooses a random polynomial $f_{i}(x) \in \mathbb{Z}_{p}[x]$ of degree $q$ and sets $h_{i}=g^{f_{i}(\alpha)}(i=1,2,3,4)$. Here, $h_{i}$ can be computed by the instance. We assume that ID $\neq \alpha$, otherwise, $\mathcal{B}_{1}$ directly solves the $q$-ABDHE problem. For ID $\neq \alpha$, set $F_{i, \mathrm{ID}}(x):=\left(f_{i}(x)-f_{i}(\mathrm{ID})\right) /(x-\mathrm{ID})$ and $\left(r_{\mathrm{ID}, i}, h_{\mathrm{ID}, i}\right):=\left(f_{i}(\mathrm{ID}), g^{F_{i, \mathrm{ID}}(\alpha)}\right)$. Then $g^{F_{i, \mathrm{ID}}(\alpha)}=g^{\left(f_{i}(\alpha)-f_{i}(\mathrm{ID})\right) /(\alpha-\mathrm{ID})}=\left(h_{i} g^{-f_{i}(\mathrm{ID})}\right)^{1 /(\alpha-\mathrm{ID})}$ hold. Since $f_{i}(x)$ is randomly chosen, the distribution of $\mathrm{dk}_{\mathrm{ID}}=\left\{\left(r_{\mathrm{ID}, i}, h_{\mathrm{ID}, i}\right)\right\}_{i=1}^{4}$ is identical to that in the actual construction. For ID* $\neq \alpha$ (if not, $\mathcal{B}_{1}$ directly solves the $q$-ABDHE problem), $\mathcal{B}_{1}$ generates $\left\{\left(r_{\mathrm{ID}^{*}, i}, h_{\mathrm{ID}^{*}, i}\right)\right\}_{i=1,2,3,4}$ as in the above. Let $\bar{f}(x)=x^{q+2}$, define a monic polynomial $\bar{F}_{\mathrm{ID}^{*}}(x):=\left(\bar{f}(x)-\bar{f}\left(\mathrm{ID}^{*}\right)\right) /\left(x-\mathrm{ID}^{*}\right)$ of (at most) $q+1$ degree, and set $\bar{F}_{\mathrm{ID}, i}$ as the $i$-th coefficient of $\bar{F}_{\mathrm{ID}^{*}}(i \in[0, q])$. Compute the source ciphertext $\bar{C}^{*}{ }^{(\kappa)}=\left(c_{1}^{*}, c_{2}^{*}, c_{3}^{*}, c_{4}^{*}, \tau^{*}\right)$ as follows:

$$
\begin{aligned}
& c_{1}^{*}:=g_{q+2}^{\prime} \cdot g^{\prime-\mathrm{ID}^{* q+2}}, c_{2}^{*}:=Z \cdot e\left(g^{\prime}, \prod_{i=0}^{q} g_{i}^{\bar{F}_{\mathrm{ID}^{*}, i}}\right), c_{3}^{*}:=M_{\beta}^{*} / e\left(c_{1}^{*}, h_{\mathrm{ID}^{*}, 1}\right) c_{2}^{* r_{\mathrm{ID}}, 1} \\
& c_{4}^{*}:=e\left(c_{1}^{*}, h_{\mathrm{ID}^{*}, 2}\right) c_{2}^{* r_{\mathrm{ID}}, 2}, c_{5}^{*}:=e\left(c_{1}^{*}, h_{\mathrm{ID}^{*}, 3} h_{\mathrm{ID}^{*}, 4}^{\delta^{*}}\right) c_{2}^{* r_{\mathrm{ID}, 3}+r_{\mathrm{ID}, 4} \delta^{*}}, \text { and } \tau^{*}:=f\left(c_{5}^{*}\right)
\end{aligned}
$$

where $\delta^{*}:=\Gamma_{h k}\left(c_{1}^{*}, c_{2}^{*}, c_{3}^{*}, c_{4}^{*}\right)$. Here, $c_{1}^{*}=g_{q+2}^{\prime} \cdot g^{\prime-\mathrm{ID}^{* q+2}}=g^{\prime \bar{f}(\alpha)-\bar{f}\left(\mathrm{ID}^{*}\right)}=g^{\left(\log _{g} g^{\prime}\right) \bar{F}_{\mathrm{ID}}(\alpha)\left(\alpha-\mathrm{ID}^{*}\right)}$ hold since $\bar{f}(\alpha)-\bar{f}\left(\mathrm{ID}^{*}\right)=\bar{F}_{\mathrm{ID}}(\alpha)\left(\alpha-\mathrm{ID}^{*}\right)$. Set $s^{*}=\left(\log _{g} g^{\prime}\right) \bar{F}_{\mathrm{ID}}(\alpha)$, and then $c_{1}^{*}=g^{s^{*}\left(\alpha-\mathrm{ID}^{*}\right)}=g_{1}^{s^{*}} \cdot g^{-s^{*} \cdot \mathrm{ID}^{*}}$. For the bit $\beta^{\prime}$ output by $\mathcal{A}, \mathcal{B}_{1}$ outputs $1\left(Z=e\left(g^{\prime}, g_{q+1}\right)\right)$ if $\beta^{\prime}=\beta$, and 0 , otherwise.

Next, we show that $\bar{C}^{*}{ }^{(\kappa)}$ is a regular ciphertext of $M_{\beta}^{*}$ as follows. Since

$$
\begin{aligned}
c_{2}^{*} & =Z \cdot e\left(g^{\prime}, \prod_{i=0}^{q} g_{i}^{\bar{F}_{\mathrm{ID}^{*}, i}}\right)=e\left(g^{\prime}, g_{q+1}\right) \cdot e\left(g^{\prime}, \prod_{i=0}^{q} g_{i}^{\bar{F}_{\mathrm{ID}}, i}\right) \\
& =e(g, g)^{\left(\log _{g} g^{\prime}\right) \alpha^{q+1}} e\left(g^{\prime}, \prod_{i=0}^{q} g^{\bar{F}_{\mathrm{ID}^{*}, i \cdot \alpha^{i}}}\right) \\
& =e(g, g)^{\left(\log _{g} g^{\prime}\right) \bar{F}_{\mathrm{ID}}(\alpha)}=e(g, g)^{s^{*}}
\end{aligned}
$$


hold,

$$
\begin{aligned}
& c_{3}^{*} / M_{\beta}^{*}=e\left(c_{1}^{*}, h_{1 \mathrm{D}^{*}, 1}\right) c_{2}^{* r_{\mathrm{ID}} *, 1} \\
& =e\left(g^{s^{*}\left(\alpha-\mathrm{ID}^{*}\right)},\left(h_{1} g^{-r_{\mathrm{ID}}^{*}, 1}\right)^{1 /\left(\alpha-\mathrm{ID}^{*}\right)}\right) e(g, g)^{s^{*} r_{\mathrm{ID}^{*}, 1}}=e\left(g, h_{1}\right)^{s^{*}}, \\
& c_{4}^{*}=e\left(c_{1}^{*}, h_{\mathrm{ID}^{*}, 2}\right) c_{2}^{* r_{\mathrm{ID}^{*}, 2}}=e\left(g, h_{2}\right)^{s^{*}},
\end{aligned}
$$

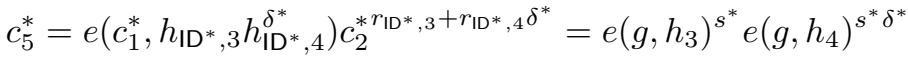

hold. Therefore, $\bar{C}^{*}(\kappa)$ is a regular ciphertext of $M_{\beta}^{*}$.

Next, we evaluate information obtained from public keys and queries. Frist, we confirm that a ciphertext with $c_{2}=e\left(c_{1}, g\right)^{1 /(\alpha-\mathrm{ID})}$ always passes the validation process in the decryption algorithm under a legitimately generated $\mathbf{d k}_{\mathrm{ID}}$ as follows. For $i=2,3,4$,

$$
\begin{aligned}
& e\left(c_{1}, h_{\mathrm{ID}, i}\right) c_{2}^{r_{\mathrm{ID}, i}}=e\left(c_{1},\left(h_{i} g^{-r_{\mathrm{ID}, i}}\right)^{1 /(\alpha-\mathrm{ID})}\right) c_{2}^{r_{\mathrm{I}, i}} \\
& =e\left(c_{1}, h_{i}\right)^{1 /(\alpha-\mathrm{ID})}\left\{e\left(c_{1}, g\right)^{1 /(\alpha-\mathrm{ID})}\right\}^{-r_{\mathrm{ID}, i}} c_{2}^{r_{\mathrm{ID}, i}} \\
& =e\left(c_{1}, h_{i}\right)^{1 /(\alpha-\mathrm{ID})} c_{2}^{-r_{\mathrm{ID}, i}} c_{2}^{r_{\mathrm{ID}, i}} \\
& =e\left(c_{1}, h_{i}\right)^{1 /(\alpha-\mathrm{ID})} \\
& =e\left(g^{s(\alpha-\mathrm{ID})}, h_{i}\right)^{1 /(\alpha-\mathrm{ID})} \\
& =e\left(g, h_{i}\right)^{s}
\end{aligned}
$$

hold. Therefore $c_{4}=e\left(c_{1}, h_{\mathrm{ID}, 2}\right) c_{2}^{r_{\mathrm{ID}, 2}}$ and $c_{5}=e\left(c_{1}, h_{\mathrm{ID}, 3} h_{\mathrm{ID}, 4}^{\delta}\right) c_{2}^{r_{\mathrm{ID}, 3}+r_{\mathrm{ID}, 4} \delta}$ hold.

Next, we show that the decryption oracle rejects a ciphertext with overwhelming probability if $c_{2} \neq$ $e\left(c_{1}, g\right)^{1 /(\alpha-\mathrm{ID})}$ holds. Let $a_{1}=\log _{g} c_{1}, a_{2}=\log _{e(g, g)} c_{2}, a_{4}=\log _{e(g, g)} c_{4}$, and $a_{5}=\log _{e(g, g)} c_{5}$, where $\delta=\Gamma_{h k}\left(c_{1}, c_{2}, c_{3}, c_{4}\right)$. If $c_{4}=e\left(c_{1}, h_{\mathrm{ID}, 2}\right) c_{2}^{r_{\mathrm{ID}, 2}}$ and $c_{5}=e\left(c_{1}, h_{\mathrm{ID}, 3} h_{\mathrm{ID}, 4}^{\delta}\right) c_{2}^{r_{\mathrm{ID}, 3}+r_{\mathrm{ID}, 4} \delta}$ hold, then

$$
\begin{aligned}
& a_{4}=a_{1} \log _{g} h_{\mathrm{ID}, 2}+a_{2} r_{\mathrm{ID}, 2} \\
& a_{5}=a_{1}\left(\log _{g} h_{\mathrm{ID}, 3}+\delta \log _{g} h_{\mathrm{ID}, 4}\right)+a_{2}\left(r_{\mathrm{ID}, 3}+\delta r_{\mathrm{ID}, 4}\right)
\end{aligned}
$$

hold. We evaluate the probability that $\mathcal{A}$ can produce such $c_{4}$ and $c_{5}$ as follows. Here, $\mathcal{A}$ knows the relations $\log _{g} h_{i}=(\alpha-\mathrm{ID}) \log _{g} h_{\mathrm{ID}, i}+r_{\mathrm{ID}, i}(i=1,2,3,4)$ from $\mathrm{dk}_{\mathrm{ID}}=\left\{\left(r_{\mathrm{ID}, i}, h_{\mathrm{ID}, i}\right)\right\}_{i=1}^{4}$. Then, we obtain the following relations:

$$
\begin{aligned}
& a_{4}=\left(a_{1} /(\alpha-\mathrm{ID})\right) \log _{g} h_{2}+\left(a_{2}-a_{1} /(\alpha-\mathrm{ID})\right) r_{\mathrm{ID}, 2} \\
& a_{5}=\left(a_{1} /(\alpha-\mathrm{ID})\right)\left(\log _{g} h_{3}+\delta \log _{g} h_{4}\right)+\left(a_{2}-a_{1} /(\alpha-\mathrm{ID})\right)\left(r_{\mathrm{ID}, 3}+\delta r_{\mathrm{ID}, 4}\right)
\end{aligned}
$$

Here, remark that $z:=a_{2}-a_{1} /(\alpha-\mathrm{ID}) \neq 0$ holds since $c_{2} \neq e\left(c_{1}, g\right)^{1 /(\alpha-\mathrm{ID})}$. Let $f_{i, j}$ be the coefficient of $x^{j}$ in $f_{i}(x)$, and $x_{k}$ be the $k$-th identity queried by $\mathcal{A}$ to the key generation oracle, and $x_{q-1}=\alpha$. Then, define the matrix $V$ by the $(q+1) \times(q-1)$ Vandermonde matrix $M_{v}$ as:

$$
V:=\left[\begin{array}{ccc}
M_{v} & \mathbf{0} & \mathbf{0} \\
\mathbf{0} & M_{v} & \mathbf{0} \\
\mathbf{0} & \mathbf{0} & M_{v}
\end{array}\right] \text { where } M_{v}:=\left[\begin{array}{cccc}
1 & 1 & \cdots & 1 \\
x_{1} & x_{2} & \cdots & x_{q-1} \\
\vdots & \vdots & \vdots & \vdots \\
x_{1}^{q} & x_{2}^{q} & \cdots & x_{q-1}^{q}
\end{array}\right]
$$

and then its columns are linearly independent. Let $\boldsymbol{f}=\left[f_{2,0}, f_{2,1}, \ldots, f_{2, q}, f_{3,0}, f_{3,1}, \ldots, f_{3, q}, f_{4,0}, f_{4,1}, \ldots, f_{4, q}\right]$ be a $3(q+1)$-degree vector, and evaluate $\boldsymbol{f} \cdot V$. From the viewpoint of $\mathcal{A}$, the solution space for $\boldsymbol{f}$ is sixdimensional since $V$ has six more rows than columns. Let $\gamma_{\mathrm{ID}}=\left(1, \mathrm{ID}, \mathrm{ID}^{2}, \ldots, \mathrm{ID}^{q}\right)$ Then, we obtain 


$$
\begin{aligned}
& a_{4}=\text { "public" terms }+z\left(\boldsymbol{f} \cdot \boldsymbol{\gamma}_{\mathrm{ID}}\|\mathbf{0}\| \mathbf{0}\right) \\
& a_{5}=\text { "public" terms }+z\left(\boldsymbol{f} \cdot \mathbf{0}\left\|\boldsymbol{\gamma}_{\mathrm{ID}}\right\| \delta \boldsymbol{\gamma}_{\mathrm{ID}}\right)
\end{aligned}
$$

where $\mathbf{0}:=(0, \ldots, 0)$ be the $(q+1)$-degree zero vector. We remark that the decryption oracle checks both $c_{4}$ and $c_{5}$ whereas the evaluation oracle checks $c_{5}$ only, and therefore accessing the evaluation oracle is better strategy from the viewpoint of $\mathcal{A}$. Actualy, $\gamma_{\mathrm{ID}}\|\mathbf{0}\| \mathbf{0}$ is in the linear span of $V$, then potentially $\mathcal{A}$ could use knowledge gained from its key generation queries to compute $a_{4}$. In the following, we assume that $\mathcal{A}$ can produce a ciphertext that passes the validation process using $c_{4}$. Then, we define a matrix $V^{\prime}$ (whose columns are linearly independent) as

$$
V^{\prime}:=\left[\begin{array}{cc}
M_{v} & \mathbf{0} \\
\mathbf{0} & M_{v}
\end{array}\right]
$$

Then, for $2(q+1)$-degree vector $\boldsymbol{f}^{\prime}=\left[f_{3,0}, f_{3,1}, \ldots, f_{3, q}, f_{4,0}, f_{4,1}, \ldots, f_{4, q}\right]$, we evaluate $\boldsymbol{f}^{\prime} \cdot V^{\prime}$. From the viewpoint of $\mathcal{A}$, the solution space for $\boldsymbol{f}^{\prime}$ is four-dimensional since $V^{\prime}$ has six more rows than columns. Then, we obtain

$$
a_{5}=\text { "public" terms }+z\left(\boldsymbol{f}^{\prime} \cdot \gamma_{\mathrm{ID}} \| \delta \gamma_{\mathrm{ID}}\right)
$$

and $\gamma_{I D} \| \delta \gamma_{I D}$ is not in the linear span of $V^{\prime}$. Therefore, for the total number of decryption/evaluation queries $Q(\ell)$, the decryption oracle rejects a ciphertext with overwhelming probability $1-Q(\ell) / p$.

Next, we evaluate the case that $Z$ is random. Then, $c_{1}^{*}$ and $c_{2}^{*}$ are uniformly random and independent. That is, $c_{2}^{*} \neq e\left(c_{1}^{*}, g\right)^{1 /\left(\alpha-\mathrm{ID}^{*}\right)}$ holds with the probability $1-1 / p$. Then,

$$
\begin{aligned}
& c_{3}^{*} / M_{\beta}^{*}=e\left(c_{1}^{*}, h_{\mathrm{ID}}, 1\right) c_{2}^{* r_{\mathrm{ID}}^{*}, 1}=e\left(c_{1}^{*},\left(h_{1} g^{-r_{\mathrm{ID}}}\right)^{1 /\left(\alpha-\mathrm{ID}^{*}\right)}\right) c_{2}^{* r_{\mathrm{ID}}^{*}, 1} \\
= & e\left(c_{1}^{*}, h_{1}\right)^{1 /\left(\alpha-\mathrm{ID}^{*}\right)}\left(c_{2}^{*} / e\left(c_{1}^{*}, g\right)^{1 /\left(\alpha-\mathrm{ID}^{*}\right)}\right)^{r_{\mathrm{ID}}, 1}
\end{aligned}
$$

hold. Moreover, since $c_{2}^{*} / e\left(c_{1}^{*}, g\right)^{1 /\left(\alpha-\mathrm{ID}^{*}\right)} \neq 1$ and $r_{\mathrm{ID}}, 1$ is uniformly random, $c_{3}^{*} / M_{\beta}^{*}$ is distributed uniformly at random. Therefore, no information of the challenge bit $\beta$ is revealed from $\left(c_{1}^{*}, c_{2}^{*}, c_{3}^{*}\right)$ as in the CPA security of the original Gentry IBE scheme.

Next, we show that the $\mathcal{A}$ 's advantage of guessing $\beta$ is at most $Q(\ell) / p$ if the decryption oracle rejects all ciphertexts with $c_{2} \neq e\left(c_{1}, g\right)^{1 /(\alpha-\mathrm{ID})}$ and are not contained in $\mathcal{D}$ as follows. For the challenge ciphertext $C^{*}=\left(c_{1}^{*}, c_{2}^{*}, c_{3}^{*}, c_{4}^{*}, \tau^{*}=f\left(c_{5}^{*}\right)\right)$, let $a_{1}=\log _{g} c_{1}^{*}, a_{2}=\log _{e(g, g)} c_{2}^{*}, a_{4}=\log _{e(g, g)} c_{4}^{*}$, and $a_{5}=\log _{e(g, g)} c_{5}^{*}$, where $\delta=\Gamma_{h k}\left(c_{1}^{*}, c_{2}^{*}, c_{3}^{*}, c_{4}^{*}\right)$. Here $a_{1}$ and $a_{2}$ are uniformly random and independent over $\mathbb{Z}_{p}$ since $Z$ is random. As in the previous discussion, $\mathcal{A}$ can obtain the relations

$$
\begin{aligned}
& \log \left(M_{\beta}^{*} / c_{3}^{*}\right) \\
& \quad=\left(a_{1} /\left(\alpha-\mathrm{ID}^{*}\right)\right) \log _{g} h_{1}+\left(a_{2}-a_{1} /\left(\alpha-\mathrm{ID}^{*}\right)\right) r_{\mathrm{ID}^{*}, 1} \\
& a_{4}=\left(a_{1} /\left(\alpha-\mathrm{ID}^{*}\right)\right) \log _{g} h_{2}+\left(a_{2}-a_{1} /\left(\alpha-\mathrm{ID}^{*}\right)\right) r_{\mathrm{ID}}, 2 \\
& a_{5}=\left(a_{1} /\left(\alpha-\mathrm{ID}^{*}\right)\right)\left(\log _{g} h_{3}+\delta \log _{g} h_{4}\right)+\left(a_{2}-a_{1} /\left(\alpha-\mathrm{ID}^{*}\right)\right)\left(r_{\mathrm{ID}^{*}, 3}+\delta r_{\mathrm{ID}}, 4\right)
\end{aligned}
$$

from the source ciphertext $\bar{C}^{*}(\kappa)$. If all irregular ciphertexts that are not contained in $\mathcal{D}$ are rejected, then no information of $r_{\mathrm{ID}^{*}, 1}$ is revealed. Moreover, key generation queries and homomorphic key reveal queries do not contain $r_{\mathrm{ID}^{*}, 1}$. Therefore, the challenge bit $\beta$ is independent to $c_{3}^{*}$ from the viewpoint of $\mathcal{A}$.

Next, we show that the decryption oracle rejects a ciphertext with $c_{2} \neq e\left(c_{1}, g\right)^{1 /(\alpha-I D)}$ with the prbability $1-Q(\ell) / p$. Let $\left(c_{1}, c_{2}, c_{3}, c_{4}, \tau=f\left(c_{5}\right), \mathrm{ID}\right) \neq\left(c_{1}^{*}, c_{2}^{*}, c_{3}^{*}, c_{4}^{*}, \tau^{*}=f\left(c_{5}^{*}\right), \mathrm{ID}{ }^{*}\right)$ be a query of $\mathcal{A}$. 
1. $\left(c_{1}, c_{2}, c_{3}, c_{4}\right)=\left(c_{1}^{*}, c_{2}^{*}, c_{3}^{*}, c_{4}^{*}\right)$ : Then, $\delta=\delta^{*}$ holds where $\delta=\Gamma_{h k}\left(c_{1}, c_{2}, c_{3}, c_{4}\right)$ and $\delta^{*}=\Gamma_{h k}\left(c_{1}^{*}, c_{2}^{*}, c_{3}^{*}, c_{4}^{*}\right)$.

- If ID $=\mathrm{ID}^{*}$, then $c_{5} \neq c_{5}^{*}$ must be hold. However, the probability that $\tau^{*}=f\left(c_{5}\right)$ is at most $\epsilon_{\mathrm{Smth}}$ due to the smoothness of $f$. Therefore, the query is rejected with overwhelming probability.

- If ID $\neq \mathrm{ID}^{*}$, then $\mathcal{A}$ needs to produce $c_{5}=e(g, g)^{a_{5}}$ which satisfies $a_{5}=$ "public" terms $+z\left(\boldsymbol{f}^{\prime}\right.$. $\left.\gamma_{\mathrm{ID}} \| \delta \gamma_{\mathrm{ID}}\right)$. However, $\mathcal{A}$ cannot generate such a $c_{5}$ except with probability $1 /(p-i+1)$ when it is the $i$-th query since the vector $\gamma_{\mathrm{ID}} \| \delta \gamma_{\mathrm{ID}}$ is linearly independent to $\gamma_{\mathrm{ID}} \| \delta^{*} \gamma_{\mathrm{ID}}$ and the columns of $V$.

2. $\left(c_{1}, c_{2}, c_{3}, c_{4}\right) \neq\left(c_{1}^{*}, c_{2}^{*}, c_{3}^{*}, c_{4}^{*}\right)$ and $\Gamma_{h k}\left(c_{1}, c_{2}, c_{3}, c_{4}\right)=\Gamma_{h k}\left(c_{1}^{*}, c_{2}^{*}, c_{3}^{*}, c_{4}^{*}\right):$ This case contradicts that $\Gamma_{h k}$ is TCR. We remark that for

$$
c_{4}^{*}=e\left(c_{1}^{*}, h_{2}\right)^{1 /\left(\alpha-\mathrm{ID}^{*}\right)}\left(c_{2}^{*} / e\left(c_{1}^{*}, g\right)^{1 /\left(\alpha-\mathrm{ID}^{*}\right)}\right)^{r_{\mathrm{ID}}, 2},
$$

$c_{2}^{*} / e\left(c_{1}^{*}, g\right)^{1 /\left(\alpha-\mathrm{ID}^{*}\right)} \neq 1$ holds since $Z$ is random. Moreover, since $r_{\mathrm{ID}^{*}, 2}$ is random, $\left(c_{1}^{*}, c_{2}^{*}, c_{3}^{*}, c_{4}^{*}\right)$ is also random over the domain of the hash function.

3. $\left(c_{1}, c_{2}, c_{3}, c_{4}\right) \neq\left(c_{1}^{*}, c_{2}^{*}, c_{3}^{*}, c_{4}^{*}\right)$ and $\Gamma_{h k}\left(c_{1}, c_{2}, c_{3}, c_{4}\right) \neq \Gamma_{h k}\left(c_{1}^{*}, c_{2}^{*}, c_{3}^{*}, c_{4}^{*}\right)$ : As in the case 1 , the query is rejected except with probability $1 /(p-i+1)$ when it is the $i$-th query.

From the above discussion, if $Z=e\left(g^{\prime}, g_{q+1}\right)$ then the source ciphertext is legitimately generated whereas if $Z$ is random then the source ciphertext is irregular. Therefore, truncated decision $q$-ABDHE problem can be solved by using whether $\mathcal{A}$ can guess the challenge bit or not.

Game $\kappa .2$ In addition to Game $\kappa .1$, replace the source ciphertext $\bar{C}^{*}(\kappa+1)$ as regular. Game $\kappa .2$ is the same as Game $\kappa+1$.

The following claim can be proved as in Claim 6.1 with the exception that the source ciphertext is computed such that $c_{3}^{*} / M=e\left(c_{1}^{*}, h_{\mathrm{ID}^{*}, 1}\right) c_{2}^{* r_{\mathrm{ID}^{*}, 1}}$ where $M$ is a random plaintext. This concludes the proof of Lemma 6.2.

Claim 6.2. $\operatorname{Pr}\left[T_{\ell}^{(\kappa .1)}\right]-\operatorname{Pr}\left[T_{\ell}^{(\kappa .2)}\right] \leq A d v_{\mathcal{A}}^{\mathrm{ABDHE}}(\ell)+A d v_{\mathcal{A}}^{\mathrm{TCR}}(\ell)+\epsilon_{\mathrm{Smth}}+Q(\ell) / p$

Concluding part of the game-hopping: Currently, no information of the challenge bit $\beta$ is contained in ciphertexts contained in $\mathcal{D}$. In this concluding part, finally, information of $\beta$ is removed from the challenge ciphertext.

Game con-0. Same as Game $Q(\ell)$.

Game con-1. In addition of Game con-0, the decryption oracle and the evaluation oracle reject all irregular ciphertexts except that the challenge ciphertext is queried to the evaluation oracle. Replace the challenge ciphertext $C^{*}$ to be irregular and $M_{\beta}^{*}$ to be a random plaintext. The following claim is proved as in Lemma 6.2 with the exception that $C^{*}$ is generated by the $q$-ABDHE instance instead of using the source ciphertext.

Claim 6.3. There exist PPT algorithms that satisfy $\operatorname{Pr}\left[T_{\ell}^{(\text {con-0) }}\right]-\operatorname{Pr}\left[T_{\ell}^{(\text {con-1) }}\right] \leq A d v_{\mathcal{B}_{1}}^{\mathrm{ABDHE}}(\ell)+A d v_{\mathcal{B}_{2}}^{\mathrm{TCR}}(\ell)$ $+\epsilon_{\mathrm{Smth}}+Q(\ell) / p$.

This concludes the proof of Theorem 6.1.

Acknowledgement: We thank the anonymous reviewers and the members of Shin-Akarui-Angou-BenkyouKai for their helpful comments. This work was supported by JSPS KAKENHI Grant Number 24700009. 


\section{References}

[1] M. Abe, J. Groth, M. Ohkubo, and M. Tibouchi. Unified, minimal and selectively randomizable structure-preserving signatures. In TCC, pages 688-712, 2014.

[2] J. H. An, Y. Dodis, and T. Rabin. On the security of joint signature and encryption. In EUROCRYPT, pages $83-107,2002$.

[3] B. Barak, O. Goldreich, R. Impagliazzo, S. Rudich, A. Sahai, S. P. Vadhan, and K. Yang. On the (im)possibility of obfuscating programs. In CRYPTO, pages 1-18, 2001.

[4] M. Barbosa and P. Farshim. Delegatable homomorphic encryption with applications to secure outsourcing of computation. In CT-RSA, pages 296-312, 2012.

[5] M. Bellare and P. Rogaway. Collision-resistant hashing: Towards making UOWHFs practical. In CRYPTO, pages 470-484, 1997.

[6] D. Bernhard, V. Cortier, O. Pereira, B. Smyth, and B. Warinschi. Adapting Helios for provable ballot privacy. In ESORICS, pages 335-354, 2011.

[7] D. Boneh, G. Segev, and B. Waters. Targeted malleability: homomorphic encryption for restricted computations. In ITCS, pages 350-366, 2012.

[8] R. Canetti, S. Halevi, and J. Katz. Chosen-ciphertext security from identity-based encryption. In EUROCRYPT, pages 207-222, 2004.

[9] R. Canetti, H. Krawczyk, and J. B. Nielsen. Relaxing chosen-ciphertext security. In CRYPTO, pages $565-582,2003$.

[10] R. Canetti, S. Raghuraman, S. Richelson, and V. Vaikuntanathan. Chosen-ciphertext secure fully homomorphic encryption. In Public-Key Cryptography, pages 213-240, 2017.

[11] D. Cash, E. Kiltz, and V. Shoup. The twin Diffie-Hellman problem and applications. In EUROCRYPT, pages $127-145,2008$.

[12] M. Chase, M. Kohlweiss, A. Lysyanskaya, and S. Meiklejohn. Malleable proof systems and applications. In EUROCRYPT, pages 281-300, 2012.

[13] R. Cramer and V. Shoup. A practical public key cryptosystem provably secure against adaptive chosen ciphertext attack. In CRYPTO, pages 13-25, 1998.

[14] R. Cramer and V. Shoup. Universal hash proofs and a paradigm for adaptive chosen ciphertext secure public-key encryption. Cryptology ePrint Archive, Report 2001/085, 2001. http://eprint .iacr.org/.

[15] R. Cramer and V. Shoup. Universal hash proofs and a paradigm for adaptive chosen ciphertext secure public-key encryption. In EUROCRYPT, pages 45-64, 2002.

[16] Y. Desmedt, R. Gennaro, K. Kurosawa, and V. Shoup. A new and improved paradigm for hybrid encryption secure against chosen-ciphertext attack. J. Cryptology, 23(1):91-120, 2010.

[17] Y. Desmedt, V. Iovino, G. Persiano, and I. Visconti. Controlled homomorphic encryption: Definition and construction. In Workshop on Encrypted Computing and Applied Homomorphic Cryptography, pages 100-122, 2017.

[18] T. ElGamal. A public key cryptosystem and a signature scheme based on discrete logarithms. IEEE Transactions on Information Theory, 31(4):469-472, 1985.

[19] K. Emura, G. Hanaoka, G. Ohtake, T. Matsuda, and S. Yamada. Chosen ciphertext secure keyedhomomorphic public-key encryption. In Public Key Cryptography, pages 32-50, 2013. 
[20] K. Emura, T. Hayashi, N. Kunihiro, and J. Sakuma. Mis-operation resistant searchable homomorphic encryption. In ASIACCS, pages 215-229, 2017.

[21] D. Galindo and J. L. Villar. An instantiation of the Cramer-Shoup encryption paradigm using bilinear map groups. Workshop on Mathematical Problems and Techniques in Cryptology, 2005.

[22] C. Gentry. Practical identity-based encryption without random oracles. In EUROCRYPT, pages 445464, 2006.

[23] C. Gentry. Fully homomorphic encryption using ideal lattices. In STOC, pages 169-178, 2009.

[24] S. Goldwasser and S. Micali. Probabilistic encryption and how to play mental poker keeping secret all partial information. In STOC, pages 365-377, 1982.

[25] J. Groth. Rerandomizable and replayable adaptive chosen ciphertext attack secure cryptosystems. In TCC, pages 152-170, 2004.

[26] G. Hanaoka and K. Kurosawa. Efficient chosen ciphertext secure public key encryption under the computational Diffie-Hellman assumption. In ASIACRYPT, pages 308-325, 2008.

[27] B. Hemenway and R. Ostrovsky. On homomorphic encryption and chosen-ciphertext security. In Public Key Cryptography, pages 52-65, 2012.

[28] D. Hofheinz and E. Kiltz. Secure hybrid encryption from weakened key encapsulation. In CRYPTO, pages 553-571, 2007.

[29] C. S. Jutla and A. Roy. Shorter quasi-adaptive NIZK proofs for linear subspaces. In ASIACRYPT, pages 1-20, 2013.

[30] C. S. Jutla and A. Roy. Dual-system simulation-soundness with applications to UC-PAKE and more. In ASIACRYPT, pages 630-655, 2015.

[31] J. Katz and V. Vaikuntanathan. Smooth projective hashing and password-based authenticated key exchange from lattices. In ASIACRYPT, pages 636-652, 2009.

[32] E. Kiltz. Chosen-ciphertext security from tag-based encryption. In TCC, pages 581-600, 2006.

[33] E. Kiltz. Chosen-ciphertext secure key-encapsulation based on gap hashed Diffie-Hellman. In Public Key Cryptography, pages 282-297, 2007.

[34] E. Kiltz, K. Pietrzak, M. Stam, and M. Yung. A new randomness extraction paradigm for hybrid encryption. In EUROCRYPT, pages 590-609, 2009.

[35] K. Kurosawa and Y. Desmedt. A new paradigm of hybrid encryption scheme. In CRYPTO, pages 426-442, 2004.

[36] J. Lai, R. H. Deng, C. Ma, K. Sakurai, and J. Weng. CCA-secure keyed-fully homomorphic encryption. In Public-Key Cryptography, pages 70-98, 2016.

[37] B. Libert, T. Peters, M. Joye, and M. Yung. Linearly homomorphic structure-preserving signatures and their applications. In CRYPTO, pages 289-307, 2013.

[38] B. Libert, T. Peters, M. Joye, and M. Yung. Non-malleability from malleability: Simulation-sound quasiadaptive NIZK proofs and CCA2-secure encryption from homomorphic signatures. In EUROCRYPT, pages 514-532, 2014.

[39] J. Loftus, A. May, N. P. Smart, and F. Vercauteren. On CCA-secure somewhat homomorphic encryption. In Selected Areas in Cryptography, pages 55-72, 2011. 
[40] P. Paillier. Public-key cryptosystems based on composite degree residuosity classes. In EUROCRYPT, pages 223-238, 1999.

[41] K. G. Paterson, J. C. N. Schuldt, M. Stam, and S. Thomson. On the joint security of encryption and signature, revisited. In ASIACRYPT, pages 161-178, 2011.

[42] M. Prabhakaran and M. Rosulek. Rerandomizable RCCA encryption. In CRYPTO, pages 517-534, 2007.

[43] M. Prabhakaran and M. Rosulek. Homomorphic encryption with CCA security. In ICALP, pages 667-678, 2008.

[44] H. Shacham. A Cramer-Shoup encryption scheme from the linear assumption and from progressively weaker linear variants. Cryptology ePrint Archive, Report 2007/074, 2007. http://eprint.iacr.org/.

[45] V. Shoup. A proposal for an ISO standard for public key encryption. Cryptology ePrint Archive, Report 2001/112, 2001. http://eprint.iacr.org/.

\section{A Smoothness of Cryptographic Functions}

In this section, we show that natural cryptographic functions, a one-way function (OWF), an always secondpreimage resistant (aSec secure) hash function [41], and a key derivation function (KDF) [16], are smooth in the sense of Definition 5.3.

Interestingly, although the amount of smoothness, $\mathrm{Smth}_{f}$, is always negligible, its "tightness" is different depending on whether the function $f$ is secure against uniform adversaries or against non-uniform adversaries. ${ }^{10}$ More specifically, for each cryptographic function $f$ considered here, we show that the smoothness of $f$ is (essentially) upperbounded by the square root of the advantage of some (uniform) PPT adversary $\mathcal{A}$ attacking the security of the function $f$. We also show that the smoothness of $f$ is (essentially) upperbounded by the advantage of some non-uniform PPT adversary $\mathrm{A}_{\text {nu. }}$. These results suggest that if we can assume the security of these cryptographic functions against non-uniform adversaries, then the output length can be as small as $\ell$-bit for $\ell$-bit security, because the smoothness of the functions are "tightly" upperbounded by the advantage of "non-uniform" adversaries attacking the security of the cryptographic functions Furthermore, even if this "non-uniform" security assumption is not justified (and instead only security against uniform adversaries is assumed), the output length the function can still be as small as at most $2 \ell$-bit, because the main term that contribute to the smoothness is the square root of the advantage of an adversary attacking the security of the cryptographic functions (against uniform PPT adversaries).

In practice, for example, (an appropriate modification of) cryptographic hash functions such as SHAseries, can be assumed to be the cryptographic functions (secure against non-uniform adversaries) considered here.

Some Notation: To show the smoothness of each cryptographic function, it is useful to introduce the following notation. Let $f: \mathcal{X}_{\ell} \rightarrow\{0,1\}^{\ell}$ be a function. For each $\ell \in \mathbb{N}$, let $y_{\ell}^{\max } \in\{0,1\}^{\ell}$ be the lexicograph-

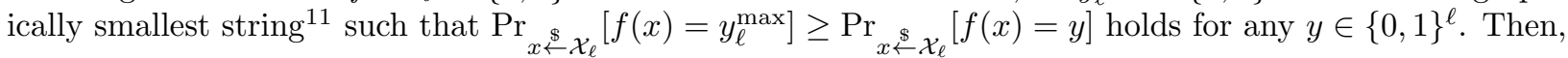
by definition, we have Smth $f=\max _{y \in\{0,1\}^{\ell}} \operatorname{Pr}_{x \aleph_{\mathcal{X}} \mathcal{X}_{\ell}}[f(x)=y]=\operatorname{Pr}_{x \leftarrow \mathcal{X}_{\ell}}\left[f(x)=y_{\ell}^{\max }\right]$. Next, for each $\ell \in \mathbb{N}$, we define $x_{\ell}^{\max } \in \mathcal{X}_{\ell}$ to be the lexicographically smallest string in the set $\left\{x \in \mathcal{X}_{\ell} \mid f(x)=y_{\ell}^{\max }\right\}$. Note that $y_{\ell}^{\max } \in\{0,1\}^{\ell}$ and $x_{\ell}^{\max } \in \mathcal{X}_{\ell}$ are uniquely determined for each $\ell \in \mathbb{N}$.

\footnotetext{
${ }^{10}$ Recall that a non-uniform algorithm is an algorithm that takes as an advice string (which is dependent only on the input length) as an additional input. The class of non-uniform PPT algorithms is equivalent to the class of polynomial-sized circuit families.

${ }^{11}$ In general, there could be multiple strings $y \in\{0,1\}^{\ell}$ that maximize the probability $\operatorname{Pr}_{x \leftarrow \mathcal{X}_{\ell}}[f(x)=y]$. Choosing the lexicographically smallest one is to canonically specify one of such strings.
} 
For the function $f$, it is also useful to note the following properties about the probability of "collision" for random inputs:

$$
\operatorname{Pr}_{x \leftarrow \mathcal{X}_{\ell}}\left[f(x)=y_{\ell}^{\max }\right]=\operatorname{Smth}_{f} \quad \text { and } \quad \underset{x, x^{\prime} \stackrel{\$}{\leftarrow} \mathcal{X}_{\ell}}{\operatorname{Pr}}\left[f(x)=f\left(x^{\prime}\right)\right] \geq\left(\text { Smth }_{f}\right)^{2},
$$

where the former is by definition, and the latter is obtained as follows:

$$
\begin{aligned}
\operatorname{Pr}_{x, x^{\prime} \leftarrow \mathcal{X}_{\ell}}\left[f(x)=f\left(x^{\prime}\right)\right] & \geq \operatorname{Pr}_{x, x^{\prime} \leftarrow \mathcal{X}_{\ell}}\left[f(x)=y_{\ell}^{\max } \wedge f(x)=y_{\ell}^{\max }\right] \\
& =\left(\underset{x \leftarrow \mathcal{X}_{\ell}}{\operatorname{Pr}}\left[f(x)=y_{\ell}^{\max }\right]\right)^{2}=\left(\text { Smth }_{f}\right)^{2}
\end{aligned}
$$

\section{A.1 One-Way Function}

Definition A.1 (One-Way Function (OWF)). Let $f: \mathcal{X}_{\ell} \rightarrow\{0,1\}^{\ell}$ be a function, where $n=n(\ell):=$ $\log _{2}\left|\mathcal{X}_{\ell}\right| \in \omega\left(\log _{2} \ell\right)$. We say that $f$ is a one-way function (OWF) if (1) $f$ is efficiently computable in terms of the security parameter $\ell$ (and thus $n$ is some polynomial of $\ell$ ), (2) we can efficiently sample an element uniformly at random from the domain $\mathcal{X}_{\ell}$, and (3) $A d v_{\mathcal{A}}^{\mathrm{OWF}}(\ell):=\operatorname{Pr}_{x \leftarrow \mathcal{X}_{\ell}}\left[x^{\prime} \leftarrow \mathcal{A}\left(1^{\ell}, f(x)\right): f\left(x^{\prime}\right)=f(x)\right]$ is negligible for any PPT algorithm $\mathcal{A}$.

Furthermore, we say that $f$ is a OWF against non-uniform adversaries if the condition (3) is replaced with "Adv ${ }_{\mathcal{A}_{\mathrm{nu}}}^{\mathrm{OWF}}(\ell)$ is negligible for any non-uniform PPT algorithms $\mathcal{A}_{\mathrm{nu}}$."

Lemma A.1. If $f$ is a $O W F$ as defined in Definition A.1, then $f$ is smooth. Specifically, there exists a PPT algorithm $\mathcal{A}$ such that

$$
\operatorname{Smth}_{f} \leq \sqrt{A d v_{\mathcal{A}}^{\mathrm{OWF}}(\ell)}
$$

Furthermore, there exists a non-uniform PPT algorithm $\mathcal{A}_{\mathrm{nu}}$ such that

$$
\operatorname{Smth}_{f}=A d v_{\mathcal{A}_{\mathrm{nu}}}^{\mathrm{OWF}}(\ell) .
$$

Proof. We first show the existence of the uniform PPT adversary $\mathcal{A}$ against the one-wayness of $f$. Consider the algorithm $\mathcal{A}$ that takes $1^{\ell}$ and $y=f(x)$ (where $x \in \mathcal{X}_{\ell}$ is chosen uniformly at random) as input, picks $x^{\prime} \in \mathcal{X}_{\ell}$ uniformly at random, and terminates with output this $x^{\prime}$. Note that $\mathcal{A}$ is a (uniform) PPT algorithm, and its one-wayness advantage is as follows:

$$
A d v_{\mathcal{A}}^{\mathrm{OWF}}(\ell)=\operatorname{Pr}_{x, x^{\prime} \leftarrow \mathcal{X}_{\ell}}\left[f(x)=f\left(x^{\prime}\right)\right] \geq\left(\mathrm{Smth}_{f}\right)^{2}
$$

where in the last step we use the inequation (3). Therefore, we have $\mathrm{Smth}_{f} \leq \sqrt{A d v_{\mathcal{A}}^{\mathrm{OWF}}(\ell)}$, as required.

We next show the existence of the non-uniform adversary $\mathcal{A}_{\text {nu }}$ against the one-wayness of $f$. Consider the non-uniform PPT algorithm $\mathcal{A}_{\text {nu }}$ that has $x_{\ell}^{\max }$ as an advice (i.e. $x_{\ell}^{\max }$ is hard-wired inside $\mathcal{A}_{\text {nu }}$ for each security parameter $\ell \in \mathbb{N}$ ), takes $1^{\ell}$ and $y=f(x)$ as input (where $x \in \mathcal{X}_{\ell}$ is chosen uniformly at random), and terminates with output the string $x_{\ell}^{\max }$. Clearly $\mathcal{A}_{\mathrm{nu}}$ is PPT, and its one-wayness advantage is:

$$
A d v_{\mathcal{A}_{\mathrm{nu}}}^{\mathrm{OWF}}(\ell)=\underset{x \leftarrow \mathcal{X}_{\ell}}{\operatorname{Pr}}\left[f(x)=f\left(x_{\ell}^{\max }\right)\right]=\underset{x \leftarrow \mathcal{X}_{\ell}}{\operatorname{Pr}}\left[f(x)=y_{\ell}^{\max }\right]=\operatorname{Smth}_{f},
$$

as required.

This completes the proof of Lemma A.1. 


\section{A.2 Always Second-Preimage Resistant Hash Functions}

Definition A.2 (Always Second-Preimage Resistant (aSec) Hash Functions [41]). Let $\mathrm{H}: \mathcal{X}_{\ell} \rightarrow\{0,1\}^{\ell}$ be a function, where $n=n(\ell):=\log _{2}\left|\mathcal{X}_{\ell}\right| \in \omega\left(\log _{2} \ell\right)$. We say that $\mathrm{H}$ is an always second-preimage resistant (aSec secure) hash function if (1) $\mathrm{H}$ is efficiently computable in terms of the security parameter $\ell$ (and thus $n$ is some polynomial of $\ell$ ), (2) we can efficiently sample an element uniformly at random from the domain $\mathcal{X}_{\ell},(3) A d v_{\mathcal{A}}^{\mathrm{aSec}}(\ell):=\operatorname{Pr}_{x \leftarrow \mathcal{X}_{\ell}}\left[x^{\prime} \leftarrow \mathcal{A}\left(1^{\ell}, x\right): \mathrm{H}\left(x^{\prime}\right)=\mathrm{H}(x) \wedge x^{\prime} \neq x\right]$ is negligible for any PPT algorithm $\mathcal{A}$.

Furthermore, we say that $\mathrm{H}$ is an aSec secure hash function against non-uniform adversaries if the condition (3) is replaced with "Adv $v_{\mathcal{A}}^{\mathrm{aSec}}(\ell)$ is negligible for any non-uniform PPT algorithm."

We remark that an aSec secure hash function is (close to but) different from the notion of universal one way hash function (UOWHF) [5]. UOWHF is a family of hash functions (or a keyed hash function), and in the security experiment, an adversary is allowed to choose the first message $x$ for which the adversary has to find a collision, but is required to find a colliding input $x^{\prime}$ under a randomly chosen key $h k$.

Lemma A.2. If $\mathrm{H}$ is an aSec secure hash function as defined in Definition A.2, then $\mathrm{H}$ is smooth. Specifically, there exists a PPT algorithm $\mathcal{A}$ such that

$$
\operatorname{Smth}_{\mathrm{H}} \leq \sqrt{A d v_{\mathcal{A}}^{\mathrm{aSec}}(\ell)+\left|\mathcal{X}_{\ell}\right|^{-1}}
$$

Furthermore, there exists a non-uniform PPT algorithm $\mathcal{A}_{\mathrm{nu}}$ such that

$$
\operatorname{Smth}_{\mathrm{H}}=A d v_{\mathcal{A}_{\mathrm{nu}}}^{\mathrm{aSec}}(\ell)+\left|\mathcal{X}_{\ell}\right|^{-1}
$$

Proof. The proof proceeds very similarly to that of Lemma A.1. First, we show the existence of the uniform PPT adversary $\mathcal{A}$ against the aSec security of $\mathrm{H}$. Consider the algorithm $\mathcal{A}$ that takes $1^{\ell}$ and $x$ (for a uniformly chosen value $x \in \mathcal{X}_{\ell}$ ) as input, picks $x^{\prime} \in \mathcal{X}_{\ell}$ uniformly at random, and terminates with output this $x^{\prime}$. Note that $\mathcal{A}$ is trivially a (uniform) PPT algorithm, and its advantage against aSec security of $\mathcal{H}$ is as follows:

$$
\begin{aligned}
A d v_{\mathcal{A}}^{\mathrm{aSec}}(\ell) & =\operatorname{Pr}_{x, x^{\prime} \stackrel{\$}{\leftarrow} \mathcal{X}_{\ell}}\left[\mathrm{H}(x)=\mathrm{H}\left(x^{\prime}\right) \wedge x \neq x^{\prime}\right] \\
& =\operatorname{Pr}_{x, x^{\prime} \stackrel{\$}{\leftarrow} \mathcal{X}_{\ell}}\left[\mathrm{H}(x)=\mathrm{H}\left(x^{\prime}\right)\right]-\operatorname{Pr}_{x, x^{\prime} \stackrel{\$}{\leftarrow} \mathcal{X}_{\ell}}\left[x=x^{\prime}\right] \\
& \geq\left(\mathrm{Smth}_{\mathrm{H}}\right)^{2}-\left|\mathcal{X}_{\ell}\right|^{-1}
\end{aligned}
$$

Therefore, we have $\operatorname{Smth}_{\mathrm{H}} \leq \sqrt{A d v_{\mathcal{A}}^{\mathrm{aSec}}(\ell)+\left|\mathcal{X}_{\ell}\right|^{-1}}$, as required.

We next show the existence of the non-uniform adversary $\mathcal{A}_{\text {nu }}$ against the aSec security of $\mathrm{H}$. Consider the non-uniform PPT algorithm $\mathcal{A}_{\text {nu }}$ that has $x_{\ell}^{\max } \in \mathcal{X}_{\ell}$ as an advice (i.e. $x_{\ell}^{\max }$ is hard-wired inside $\mathcal{A}_{\text {nu }}$ for each security parameter $\ell \in \mathbb{N}$ ), takes $1^{\ell}$ and $x$ as input (where $x$ is chosen uniformly at random), and terminates with output the string $x_{\ell}^{\max }$. Clearly $\mathcal{A}_{\mathrm{nu}}$ is PPT, and its advantage is:

$$
\begin{aligned}
A d v_{\mathcal{A}_{\mathrm{nu}}}^{\mathrm{aSec}}(\ell) & =\underset{x \stackrel{\$}{\leftarrow} \mathcal{X}_{\ell}}{\operatorname{Pr}}\left[\mathrm{H}(x)=\mathrm{H}\left(x_{\ell}^{\max }\right) \wedge x \neq x_{\ell}^{\max }\right] \\
& =\underset{x \stackrel{\$}{\leftarrow} \mathcal{X}_{\ell}}{\operatorname{Pr}}\left[\mathrm{H}(x)=y_{\ell}^{\max }\right]-\underset{x \stackrel{\$}{\leftarrow} \mathcal{X}_{\ell}}{\operatorname{Pr}}\left[x=x_{\ell}^{\max }\right] \\
& =\operatorname{Smth}_{\mathrm{H}}-\left|\mathcal{X}_{\ell}\right|^{-1},
\end{aligned}
$$

Therefore, we have $\operatorname{Smth}_{\mathrm{H}}=A d v_{\mathcal{A}_{\mathrm{nu}}}^{\mathrm{aSec}}(\ell)+\left|\mathcal{X}_{\ell}\right|^{-1}$, as required.

This completes the proof of Lemma A.2. 


\section{A.3 Key Derivation Function}

Definition A.3 (Key Derivation Function (KDF) [16]). Let KDF : $\mathcal{X}_{\ell} \rightarrow\{0,1\}^{\ell}$ be a function, where $n=n(\ell):=\log _{2}\left|\mathcal{X}_{\ell}\right| \in \omega\left(\log _{2} \ell\right)$. We say that KDF is a secure key derivation function (KDF) if (1) KDF is efficiently computable in terms of the security parameter $\ell$ (and thus $n$ is some polynomial of $\ell$ ), (2) We can efficiently sample an element uniformly at random from the domain $\mathcal{X}_{\ell}$, and $(3) \operatorname{Adv}_{\mathcal{A}}^{\mathrm{KDF}}(\ell):=$ $\left|\operatorname{Pr}_{x \leftarrow \Delta}\left[\mathcal{A}\left(1^{\ell}, \operatorname{KDF}(x)\right)=1\right]-\operatorname{Pr}_{y \stackrel{\$}{\leftarrow}\{0,1\}^{\ell}}\left[\mathcal{A}\left(1^{\ell}, y\right)=1\right]\right|$ is negligible for any PPT algorithm $\mathcal{A}$.

Furthermore, we say that $\mathrm{KDF}$ is a secure KDF against non-uniform adversaries if $A d v_{\mathcal{A}_{\mathrm{nu}}}^{\mathrm{KDF}}(\ell)$ is negligible for any non-uniform algorithm $\mathcal{A}_{\mathrm{nu}}$.

Lemma A.3. If KDF be a secure key derivation function as defined in Definition A.3, then KDF is smooth. Specifically, there exists a uniform PPT algorithm $\mathcal{A}$ such that

$$
\mathrm{Smth}_{\mathrm{KDF}} \leq \sqrt{A d v_{\mathcal{A}}^{\mathrm{KDF}}(\ell)+2^{-\ell}}
$$

Furthermore, there exists a non-uniform PPT algorithm $\mathcal{A}_{n u}$ such that

$$
\operatorname{Smth}_{\mathrm{KDF}}=A d v_{\mathcal{A}_{\mathrm{nu}}}^{\mathrm{KDF}}(\ell)+2^{-\ell} .
$$

Proof. We first show the existence of the uniform PPT adversary $\mathcal{A}$ against the security of KDF. Consider the algorithm $\mathcal{A}$ that takes $1^{\ell}$ and $y \in\{0,1\}^{\ell}$ as input, picks $x^{\prime} \in \mathcal{X}_{\ell}$ uniformly at random, and returns 1 if $G\left(x^{\prime}\right)=y$ or returns 0 otherwise. Note that $\mathcal{A}$ is clearly PPT, and its advantage is as follows:

$$
\begin{aligned}
& A d v_{\mathcal{A}}^{\mathrm{KDF}}(\ell)=\left|\operatorname{Pr}_{x \notin \mathcal{X}_{\ell}}\left[\mathcal{A}\left(1^{\ell}, \operatorname{KDF}(x)\right)=1\right]-\operatorname{Pr}_{y \stackrel{\$}{\leftarrow}\{0,1\}^{\ell}}\left[\mathcal{A}\left(1^{\ell}, y\right)=1\right]\right| \\
& =\left|\operatorname{Pr}_{x, x^{\prime} \stackrel{\$}{\leftarrow} \mathcal{X}_{\ell}}\left[\operatorname{KDF}(x)=\operatorname{KDF}\left(x^{\prime}\right)\right]-\operatorname{Pr}_{y \stackrel{\$}{\leftarrow}\{0,1\}^{\ell}, x^{\prime} \stackrel{\$}{\leftarrow} \mathcal{X}_{\ell}}\left[y=\operatorname{KDF}\left(x^{\prime}\right)\right]\right| \\
& \geq\left(\mathrm{Smth}_{\mathrm{KDF}}\right)^{2}-2^{-\ell},
\end{aligned}
$$

where in the last inequality we use the inequality (3) and the fact that $y$ is chosen uniformly at random from $\{0,1\}^{\ell}$. Therefore, we have $\operatorname{Smth}_{\mathrm{KDF}} \leq \sqrt{A d v_{\mathcal{A}}^{\mathrm{KDF}}(\ell)+2^{-\ell}}$, as required.

Next, we show the existence of the non-uniform PPT adversary $\mathcal{A}_{\text {nu }}$ against the security of KDF. Consider the algorithm $\mathcal{A}_{\text {nu }}$ that has $y_{\ell}^{\max } \in\{0,1\}^{\ell}$ as an advice (i.e. $y_{\ell}^{\max }$ is hard-wired inside $\mathcal{A}_{\text {nu }}$ for each $\ell \in \mathbb{N}$ ), takes $1^{\ell}$ and $y \in\{0,1\}^{\ell}$ as input, and returns 1 if $y=y_{\ell}^{\max }$ or returns 0 otherwise. Note that $\mathcal{A}_{\text {nu }}$ is clearly $\mathrm{PPT}$, and its advantage is as follows:

$$
\begin{aligned}
& A d v_{\mathcal{A}_{\mathrm{nu}}}^{\mathrm{KDF}}(\ell)=\left|\operatorname{Pr}_{x \leftarrow \mathcal{X}_{\ell}}\left[\mathrm{A}_{\mathrm{nu}}\left(1^{\ell}, \operatorname{KDF}(x)\right)=1\right]-\operatorname{Pr}_{y \stackrel{\$}{\leftarrow}\{0,1\}^{\ell}}\left[\mathrm{A}_{\mathrm{nu}}\left(1^{\ell}, y\right)=1\right]\right| \\
& =\left|\operatorname{Pr}_{x \leftarrow \mathcal{X}_{\ell}}\left[\operatorname{KDF}(x)=y_{\ell}^{\max }\right]-\operatorname{Pr}_{y \stackrel{\$}{\leftarrow}\{0,1\}^{\ell}}\left[y=y_{\ell}^{\max }\right]\right| \\
& =\operatorname{Smth}_{\mathrm{KDF}}-2^{-\ell} \text {. }
\end{aligned}
$$

Therefore, we have $\mathrm{Smth}_{\mathrm{KDF}}=A d v_{\mathcal{A}_{\mathrm{nu}}}^{\mathrm{KDF}}(\ell)+2^{-\ell}$, as required.

This completes the proof of Lemma A.3. 